\title{
Broadband and Polarization Flexible SOI Grating Coupler based on Sub-Wavelength Gratings with Low Back Reflections
}

\author{
by
}

Neha Purwaha

B. Tech., P.G.D.

\begin{abstract}
A thesis submitted to the
Faculty of Graduate and Postdoctoral Affairs

in partial fulfillment of the requirements for the degree of
\end{abstract}

Master of Applied Science in Electrical and Computer Engineering

Department of Electronics

Carleton University

Ottawa, Ontario

December 2018

(C) Copyright

Neha Purwaha, 2018 


\section{Abstract}

Optical couplers play an integral role in coupling of the guided mode from an optical fiber into a Photonic Integrated Circuit (PIC) and vice-versa. Apart from the edge couplers, grating couplers are the most extensively used optical I/O's. While edge couplers couple light within the same plane, grating couplers diffract the inplane guided mode into an out-of-plane radiated beam, thereby changing the direction of propagation. Though grating couplers are easy to fabricate and are wellsuited for wafer-scale testing, they have limited application owing to their polarization selectivity and a coupling bandwidth of only $30-40 \mathrm{~nm}$. This restricts the use of grating couplers in Dense Wavelength Division Multiplexing (DWDM) applications.

To overcome, the shortcomings of a conventional grating coupler, we first investigated the fundamentals of grating coupler and studied the principle governing polarization insensitivity for a surface grating coupler. By engineering the average effective index of the grating coupler using sub-wavelength gratings, we increased the coupling bandwidth while maintaining polarization insensitivity. A broadband grating coupler can help eliminate the bottleneck in the coupling of DWDM fibers to the PICs. A polarization flexible grating coupler can realize higher transmission rates by introducing polarization diversity (TE and TM polarization operate independently) thereby resulting in increased data capacity of the Optical Telecommunication Network (OTN). With a uniform sub-wavelength grating coupler (SWGC) designed in this thesis, we attained 3-dB operating bandwidth of $105 \mathrm{~nm}$ and $121 \mathrm{~nm}$ for the 
TE and TM polarization, respectively. The proposed device attains coupling efficiency of $-4.88 \mathrm{~dB}$ for both TE and TM polarization at $1550 \mathrm{~nm}$, resulting in near zero polarization dependent loss. A 1-dB coupling bandwidth of $57 \mathrm{~nm}$ was obtained for TE polarization, whereas TM attained an even higher bandwidth of 69 $\mathrm{nm}$. To the best of our knowledge, the SWGC design proposed in this thesis offers the best reported performance in bandwidth and polarization sensitivity. 


\section{Acknowledgement}

Firstly, I would like to thank my mentor and supervisor, Professor Dr. Winnie N. Ye for giving me the opportunity of being a part of her research group and working under her supervision. She has been a constant source of guidance and motivation throughout this project. I would also like to extend my gratitude to my colleagues Niharika Kohli, Dusan Gostimirovic and Vladyslav Vakarin for sharing their knowledge, expertise and valuable insight with me. And, to my friends and roommates for all the fun-times and supporting me through the entire process. I am very thankful to my family for being my pillars of strength throughout my degree program and helping me become what I'm today. 


\section{Table of Contents}

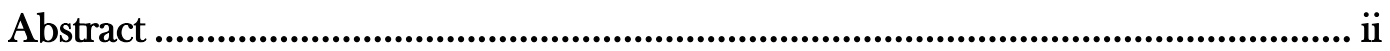

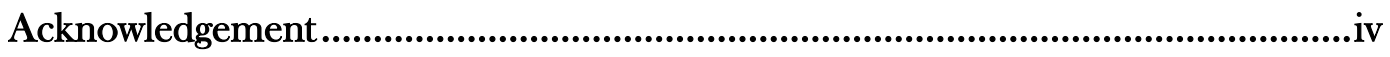

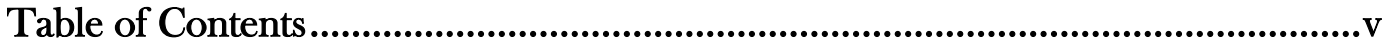

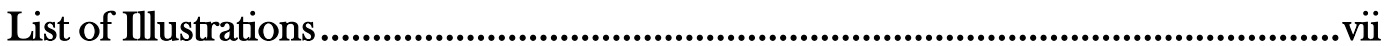

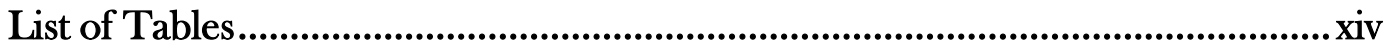

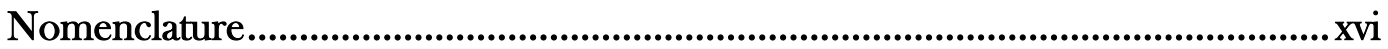

Chapter 1 Introduction........................................................................................ 1-1

Chapter 2 Literature Review................................................................................. 2-8

2.1 Broadband Grating Couplers .......................................................................... 2-8

2.2 Polarization Independent Grating Couplers ................................................2-14

Chapter 3 Grating Coupler Fundamentals ....................................................... 3-24

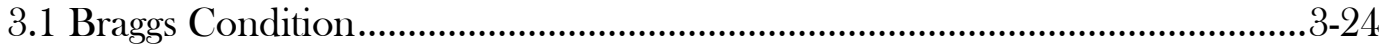

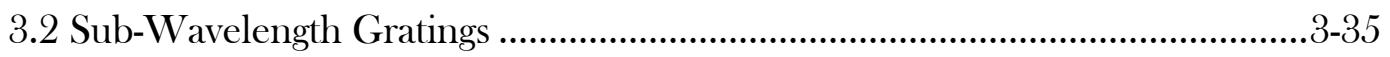

3.3 Grating Optimizations: Apodized and Focusing Grating ..............................3-36

Chapter 4, Computational Methods ................................................................. 4-40

4.1 Effective Index Approximation ..................................................................4-40

4.2 Effective Medium Theory......................................................................4-45 
4.3 Finite Difference Time Domain Method ......................................................4-50

Chapter 5 Polarization Flexible and Broadband Grating Design....................... 5-54

5.1 Designing Uniform Sub-Wavelength Grating Coupler ...................................5-55

5.2 Designing Apodized Sub-Wavelength Grating Coupler..................................5-92

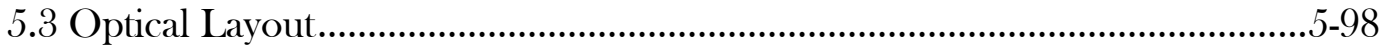

Chapter 6 Conclusions ............................................................................... 6-102

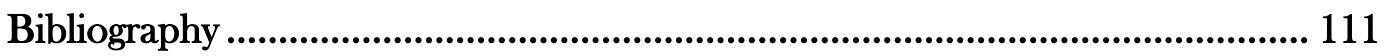

Appendix A Partial Etched GC Setup and 2D-FDTD Simulation Scripts .......... 116

Appendix B SWGC Device Setup and 2D-FDTD Simulation Scripts .............. 120

Appendix C Analysis Scripts ...................................................................... 124 


\section{List of Illustrations}

1. Figure 1.1. Cross-section of a (a) Single Mode Fiber and (b) $500 \mathrm{~nm}$ wide planar silicon waveguide ......................................................................

2. Figure 1.2. Illustration for the device setup for edge coupling (Ref. [3]) ....1-4

3. Figure 1.3. A typical setup for surface coupling between fiber and planar waveguide using grating couplers (Ref. [10]) ............................................ 1-5

4. Figure 2.1. Schematic of the cross section of the SWGC with onedimensional subwavelength gratings; (b) schematic of and SWGC with refractive index regions of $n_{н}$ and $n_{\mathrm{L}}$ (Wang et al.)

5. Figure 2.2. Simulated 1-dB bandwidth and peak $\mathrm{CE}$ as a function of $\mathrm{ff}_{\mathrm{L}}$ for the SWGC designed by Wang et al.

6. Figure 2.3. SEM images of the as-fabricated focusing and straight SWGC for design reported in Ref. [11]

7. Figure 2.4. Schematic Illustration of the broadband grating coupler design proposed in [12]. 2-11

8. Figure 2.5. Transmission spectrum for the simulated device in [12].......2-11

9. Figure 2.6. SEM image of the fabricated focusing-curved SWGC fabricated and demonstrated in [12]

10. Figure 2.7. Measured transmission spectrum of the focusing-curved SWGC tapered grating design (Ref. [12]) 
11. Figure 2.8. Simulated transmission and reflection spectra of the simulated device in [11] . -13

12. Figure 2.9. Transmission spectrum of the design reported in [11] reproduced using Lumerical FDTD Solutions for straight waveguide

13. Figure 2.10.Proposed structure by Tang and Dai served as both a polarization splitter and a vertical [14]

14. Figure 2.11. Schematic of an apodized focusing SWG for coupling two polarization into a single SMW (Ref. [15]) 2-16

15. Figure 2.12. Coupling efficiency of the apodized SWG designs using 2D FDTD simulation (Ref. [15]) 2-17

16. Figure 2.13 (a) Scanning electron microscope (SEM) image of the apodized focusing SWGs. (b) SEM image of the cross section of a SMW (Ref. [15]) 217

17. Figure 2.14. Measurements of apodized focusing SWGs with different fillfactors of the sub-wavelength structure designed in [15] 2-18

18. Figure 2.15. Schematic of the nonuniform grating period formation (a) intersection and (b) union of TE and TM mode grating periods. (Ref. [35] )

19. Figure 2.16. Fiber coupling performance of optimized (a) intersection and (b) union grating couplers (Ref. [35]).

20. Figure 2.17. Wavelength dependence of the coupling efficiency for the optimized device ( Ref. [14]) 
21. Figure 2.18. Re-generated results from [14], using Lumerical FDTD .....2-21

22. Figure 3.1. Scattering of plane wave from a periodic medium .................3-25

23. Figure 3.2. Simple grating coupler side view $.3-26$

24. Figure 3.3. Momentum Diagram of Forward Radiation mode into the air/cladding (Ref. [16])

25. Figure 3.4. Overlap between the radiated beam and the Gaussian fiber mode

26. Figure 3.5. Schematic representation of grating couplers a) uniform grating b) based upon duty cycle apodization c) based on SWG apodization c) based on etch-depth apodization (Ref. [24]) 3-36

27. Figure 4.1 Schematic diagram depicting geometry of (a) Strip waveguide (b) Rib waveguide $4-41$

28. Figure 4.2. Mode profiles of the two guided mode in strip waveguide (a) Electric field intensity profile for Mode-1 (b) Magnetic field intensity profile for Mode-1 (a) Electric field intensity profile for Mode-2 (b) Magnetic field intensity of Mode-2 .................................................................................. 4-42

29. Figure 4.3. (a) General diagram of strip waveguide (b)- (c) decomposition into two imaginary planar waveguides $($ Ref.[21]) .............................................4-43

30. Figure 4.4. Effective Index for TE polarization (Ref. [34] ) .....................4-46

31. Figure 4.5. Effective Index for TM Polarization (Ref. [34]) ......................4-48

32. Figure 4.6. (a) Effective indices for TE and TM polarizations in a (a) high dielectric contrast medium (b) low dielectric contrast medium................4-50 
33. Figure 4.7. FDTD mesh for a grating coupler $.4-53$

34. Figure 5.1. Schematic diagram for the polarization independent grating coupler design $5-57$

35. Figure 5.2. Schematic for the 2D simulation of the grating coupler.........5-58

36. Figure 5.3. Transmission spectrum for the initial grating design ..............5-59

37. Figure 5.4. Variation of Transmission with fill-factor of grating for TE polarized light. $5-60$

38. Figure 5.5. Variation of Transmission with fill-factor of grating for TM polarized light $5-61$

39. Figure 5.6. Variation of maximum transmission and wavelength with fillfactor $5-61$

40. Figure 5.7. Variation of Transmission with Grating period for TE polarized light $5-63$

41. Figure 5.8. Variation of Transmission with Grating period for TM polarized light $5-63$

42. Figure 5.9. Variation of maximum transmission and wavelength with grating period 5-64

43. Figure 5.10. Variation of Transmission with etch depth for TE polarized light 5-65

44. Figure 5.11. Variation of Transmission with etch depth for TM polarized light .5-66 
45. Figure 5.12. Variation in the maximum transmission and coupling wavelength with etch depth. $5-66$

46. Figure 5.13. Transmission spectrum for TE and TM of the optimized polarization independent design ......................................................5-68

47. Figure 5.14. Coupling efficiency and bandwidths for TE and TM...........5-69

48. Figure 5.15. S-parameter for the TE polarization ..................................5-70

49. Figure 5.16. S-parameter for the TM polarization ..................................5-71

50. Figure 5.17. Plot for equation (5.9) …..................................................5-73

51. Figure 5.18. Sub-wavelength structure in high and low index regions ......5-74

52. Figure 5.19. Transmission spectrum for the initial SWGC .....................5-75

53. Figure 5.20. Variation of transmission spectrum with respect to grating period of SWGC for TE polarization $5-76$

54. Figure 5.21. Variation of transmission spectrum with respect to grating period of SWGC for TM polarization $5-77$

55. Figure 5.22. Variation of transmission spectrum for TE polarization with duty cycle of the grating period. $5-78$

56. Figure 5.23. Variation of transmission spectrum for TM polarization with duty cycle of the grating period $5-78$

57. Figure 5.24. Variation of the transmission spectrum with fill-factor (ffL) for TE polarized mode $5-80$

58. Figure 5.25. Variation of the transmission spectrum with fill-factor (ffL) for TM polarized mode $5-80$ 
59. Figure 5.26. Variation of Transmission spectrum with number of periods $\left(\mathrm{N}_{\mathrm{L}}\right)$ in the low index region for TE polarization $5-81$

60. Figure 5.27. Variation of the Transmission spectrum with number of periods $\left(\mathrm{N}_{\mathrm{L}}\right)$ in the low index region for TM polarization. $5-82$

61. Figure 5.28. Schematic diagrams for the sub-wavelength grating coupler (SWGC) (a) Top View (b) Cross-section view with effective index approximation (c) 3D representation. $5-83$

62. Figure 5.29. Simulation setup for a SWGC. $5-85$

63. Figure 5.30. Normalized transmission spectrum for polarization flexible and broadband grating coupler. $5-87$

64. Figure 5.31. Coupling efficiency of the polarization flexible and broadband grating coupler. $5-88$

65. Figure 5.32. Coupling bandwidths for the polarization flexible and broadband grating $5-88$

66. Figure 5.33. Coupling efficiency of the polarization flexible and broadband grating coupler using 3D- FDTD Simulation

67. Figure 5.34. Variation of Transmission with respect to periods in the low index region. $5-90$

68. Figure 5.35. S-Parameter of the Polarization flexible and broadband SWGC $5-92$

69. Figure 5.36. Apodized SWGC for polarization flexible and broadband operation 
70. Figure 5.37. Transmission spectrum for the apodized SWGC

71. Figure 5.38. Coupling efficiency of the apodized SWGC.

72. Figure 5.39. S-Parameter for the apodized SWGC for TE and TM polarization.

73. Figure 5.40. Optical layout for (a) the straight polarization flexible and broadband grating coupler; (b) Close-up view of the sub-wavelength gratings

74. Figure 5.41. Optical layout for (a) focusing polarization flexible and broadband grating coupler; (b) Close-up view of the sub-wavelength gratings

75. Figure 5.42. Optical layout for uniform SWGC design for fabrication (a) focusing; (b) Close-up view of the sub-wavelength gratings $5-100$

76. Figure 5.43. Optical layout for (a) focusing apodized polarization flexible and broadband SWGC; (b) Close-up view of the gratings ...........................5-101 


\section{List of Tables}

1. Table 2-1. State-of-the-art grating couplers

2. Table 3-1. Dependence of $\beta$ on the grating period (Ref. [16])

3. Table 5-1. Effective indices for the TE and TM polarization at $1550 \mathrm{~nm} . .5$ 56

4. Table 5-2. Initial grating coupler parameters.........................................5-58

5. Table 5-3. Optimized design parameters for partially etched polarization independent and splitter design $5-67$

6. Table 5-4. Simulation Results $5-68$

7. Table 5-5 Initial sub-wavelength grating design $5-74$

8. Table 5-6. Optimized design parameters for the polarization flexible and broadband grating coupler design $5-85$

9. Table 5-7. Simulation results for polarization flexible and broadband grating design using 2D -FDTD $5-86$

10. Table 5-8 Simulation results for polarization flexible and broadband grating design using 3D- FDTD 5-86

11. Table 5-9. Optimized design parameters for the fabrication of polarization flexible and broadband grating coupler design .....

12. Table 5-10. Simulation results for design for fabrication of polarization flexible and broadband grating design 
13. Table 5-11. Design parameters for broadband and polarization flexible apodized SWGC

14. Table 5-12. Simulation results for broadband and polarization flexible apodized SWGC 


\section{Nomenclature}

\section{List of Abbreviations}

\begin{tabular}{|l|l|}
\hline Notation & Description \\
\hline SOI & Silicon-on-Insulator \\
\hline GC & Grating Coupler \\
\hline SMF & Single Mode Fiber \\
\hline MMF & Multi-mode Fiber \\
\hline TE & Transverse Electric \\
\hline TM & Transverse Magnetic \\
\hline PIC & Photonic Integrated Circuit \\
\hline SWGC & Sub-Wavelength Grating Coupler \\
\hline EMT & Effective Medium Theory \\
\hline CE & Coupling Efficiency \\
\hline FDTD & Finite-Difference Time-Domain \\
\hline
\end{tabular}




\section{List of Symbols}

\begin{tabular}{|l|l|}
\hline Notation & Description \\
\hline $\boldsymbol{\theta}$ & Angle of incidence of the fiber \\
\hline $\mathbf{n}_{\mathbf{e f f}}$ & Effective index \\
\hline $\mathbf{n}_{\mathbf{c}}$ & Effective index of cladding \\
\hline $\boldsymbol{\lambda}$ & Wavelength \\
\hline ed & Etch Depth \\
\hline tSi & Height of silicon on SOI \\
\hline $\mathbf{f f}$ & Fill-Factor of grating \\
\hline $\mathbf{f f}$ & Fill-factor of low-index region \\
\hline $\mathbf{f f H}$ & Fill-factor of high-index region \\
\hline
\end{tabular}




\section{Chapter 1}

\section{Introduction}

\subsection{Silicon Photonics}

Silicon Photonics has attracted tremendous research interest throughout the past decade. Although with many outstanding optical and electrical properties, there are still certain challenges in this domain that are yet to be addressed. One of the key challenges is efficient coupling of light from a fiber into and out of photonic integrated circuits (PICs). A conventional silicon waveguide has a cross-sectional area of the order of $10^{-1} \mu \mathrm{m}^{2}$, while a single mode fiber (SMF) has a core diameter of $\sim 8.2 \mu \mathrm{m}$ (as seen in Figure 1.1) resulting in a mode field diameter (MFD) $\sim 9 \mu \mathrm{m}$.

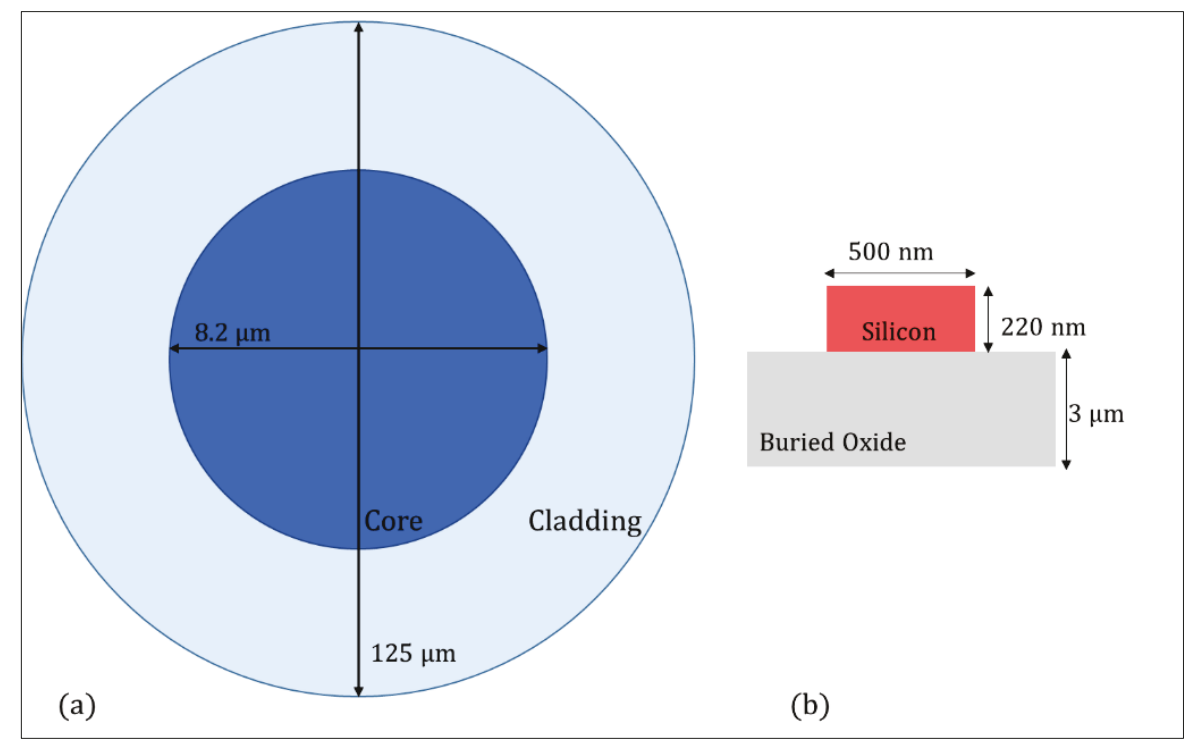

Figure 1.1. Cross-section of a (a) Single Mode Fiber and (b) $500 \mathrm{~nm}$ wide planar silicon waveguide 
With the increasing demand for higher data capacity at a faster speed, the optical network for data communication and telecommunication are adopting Dense Wavelength Division Multiplexing(DWDM) which can presently carry up to 96 data channels per fiber with a standard channel spacing of $0.8 \mathrm{~nm}$ or $0.4 \mathrm{~nm}$ [1], leading to a bandwidth requirement of $\sim 80 \mathrm{~nm}$.DWDM has enabled a single fiber to be able to transmit data at speeds up to $400 \mathrm{~Gb} / \mathrm{s}$. As the technology advances, the DWDM fiber may support up to as high as 128 channels, increasing the minimum bandwidth limit to almost $100 \mathrm{~nm} \mathrm{[2]} \mathrm{and} \mathrm{resulting} \mathrm{in} \mathrm{an} \mathrm{overall} \mathrm{higher} \mathrm{data} \mathrm{rate.}$ Thus, it is highly desirable to employ an optical coupler that is efficient as well as broadband. The C-band consist of wavelength ranges from 1530-1565 nm, resulting in a bandwidth of only $35 \mathrm{~nm}$. For broadband application we require at least $100 \mathrm{~nm}$ bandwidth at a central operating wavelength of $1550 \mathrm{~nm}$, thereby invading parts of the S-band (1460-1530 nm) and the L-band (1565-1625 nm). The improvement in data capacity can be realized by either increasing the number of data channels, or through the introduction polarization diversity, where TE and TM polarization operate independently. By establishing polarization insensitivity to the optical couplers, the data rate of the PICs effectively doubles. Therefore, an optical coupler that is both polarization flexible and broadband in operation is highly desirable for high-data rate telecom applications. A brief overview of the most popular fiberwaveguide coupling techniques and their characteristic properties is given in the next section. 


\subsection{Fiber- Planar Waveguide Coupling Techniques}

The two most widely employed coupling techniques for efficiently coupling a guided mode from a fiber into a planar waveguide are-1) Edge coupling 2) Surface Coupling (using grating couplers). In this section we familiarize ourselves with the two coupling techniques followed by a comparison of their performance characteristics. The performance of the optical couplers are completely described by three factors, which are- Coupling Efficiency (CE), Bandwidth and Back-Reflections, these are further detailed in Section 3.1.4 . CE is measured in $\mathrm{dBs}$ and is described as the ratio of the power incident from the first waveguide to the power that is coupled into the subsequent waveguide. Back-Reflection is again denoted in $\mathrm{dBs}$ and is described as the ratio of the power that incident from the first waveguide to the power reflected into the same waveguide caused by the refractive index contrast. The 1-dB or 3-dB bandwidths are described as the continuous range of wavelengths for which the $C E$ at that wavelength is such that, $C E \geq C E_{\max }-1$ or $C E \geq C E_{\max }-3$, respectively.

\subsubsection{Edge Coupling}

A typical setup for edge coupling is illustrated in Figure 1.2. An edge coupler consists of a silicon inverse taper, where the waveguide width at the edge of SOI wafer is reduced so that the waveguide is in the cut-off condition and a valid mode does not exists. Under these conditions, the mode in the planar waveguide broadens and spreads into the cladding. 


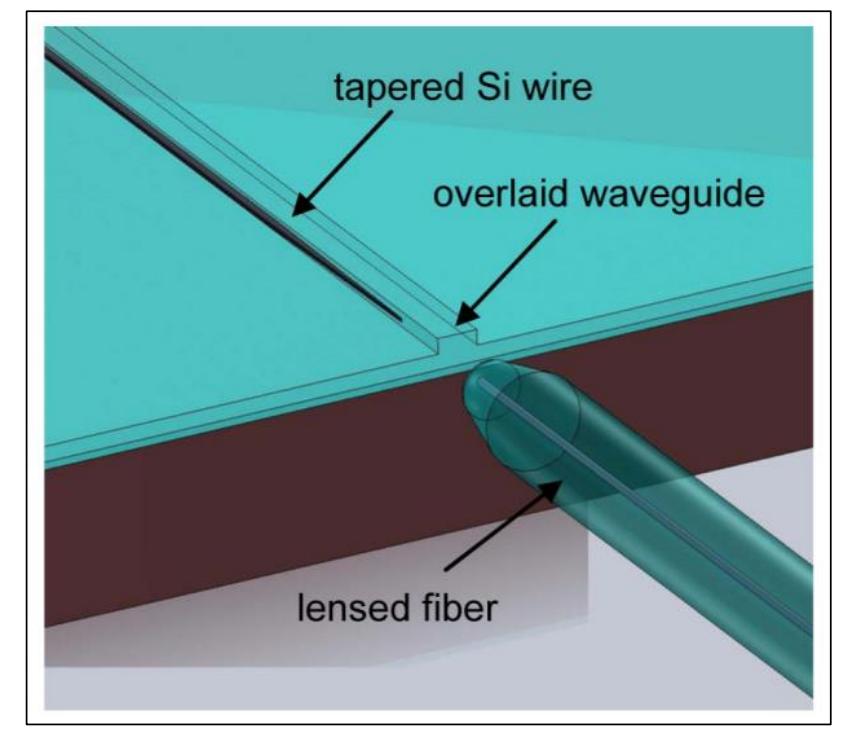

Figure 1.2. Illustration for the device setup for edge coupling (Ref. [3])

This results in an MFD of size similar to that of a fiber, thereby resulting in efficient fiber-waveguide coupling. Experimental demonstrations of sophisticated edge couplers measured coupling losses less than $0.25 \mathrm{~dB}$ at central wavelength of $1550 \mathrm{~nm}$ with 1-dB coupling bandwidths as high as $100 \mathrm{~nm}$ for both TE and TM polarizations and 3-dB bandwidth of almost $300 \mathrm{~nm}$ [3]. However, edge couplers have their own set of constraints. To begin with, they require perfectly polished lensed fibers for efficient coupling of light from the fiber into an inverse taper of lateral widths which may be as small as $80 \mathrm{~nm}$ [4]; also, they require long tapers for the adiabatic conversion of a micrometric mode to a nano-sized mode with lengths of the order of hundreds of microns [5]- [7],; and thirdly, they require high-resolution optical alignment, which increases the packaging cost [8]. Additionally, the edge 
couplers can only be placed along the edge of a PIC, which adds additional complexity to their post-fabrication facet preparation and testing.

\subsubsection{Grating Couplers}

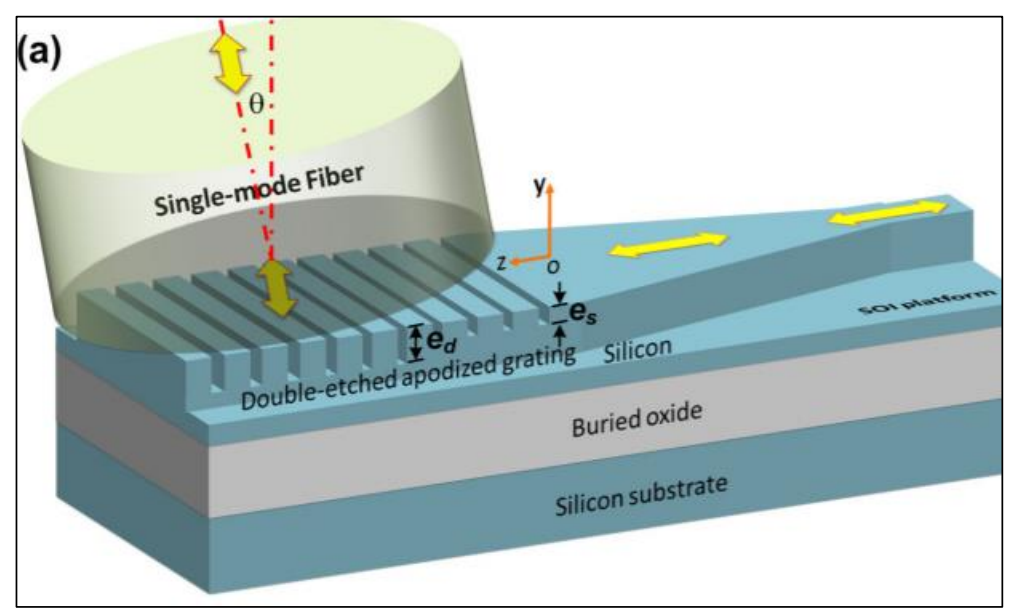

Figure 1.3. A typical setup for surface coupling between fiber and planar waveguide using grating couplers (Ref. [10])

Grating couplers are surface devices with length and width approximately equal to the MFD of the single mode fiber. The device setup of a typical grating coupler is illustrated in Figure 1.3. Grating couplers are periodic structures of alternating regions of high and low index layers. In a typical grating design, the high index region is the unetched silicon and the low-index region maybe formed by partially or fully etched silicon. These gratings pass on the coupled mode to a $12 \mu \mathrm{m}$ wide waveguide which is then adiabatically tapered into a $500 \mathrm{~nm}$ wide silicon nanowire. Owing to the larger effective area of interaction between the grating coupler and the fiber, the grating couplers have greater alignment tolerance. Grating couplers also have a higher fabrication tolerance with the flexibility of placement on the wafer giving 
them an advantage over edge couplers for wafer-scale testing. However, they also have their set of limitations- 1) they are intrinsically polarization selective as a result of the grating parameters and normally allow only one polarization to be coupled in; 2) they have lower operating bandwidths as compared to edge couplers; 3) they also have lower coupling efficiency (CE) than edge couplers because of the power lost towards the substrate. Further decrease in coupling efficiency is caused by the mismatch in the Gaussian profile of the fiber mode and the radiated mode of the grating. Though, 1-dB bandwidth of $30-40 \mathrm{~nm}$ is sufficient for single-channel ICT systems, it prevents the usage of the conventional grating couplers in multi-channel ICT systems that rely on DWDM, where a bandwidth up to 80-100 nm is required [9]. Hence, it is necessary to devise an optical coupler which exhibits large bandwidth and polarization insensitivity, along with ease of fabrication and integration, to accommodate the high data capacities of DWDM applications.

\subsection{Thesis Overview}

With these requirements, the objective of this thesis is to realize a polarization flexible and broadband grating coupler with 3-dB coupling bandwidth greater than $100 \mathrm{~nm}$ at a central operating wavelength of $1550 \mathrm{~nm}$. We start with a literature survey on the state-of-the-art polarization independent and broadband grating couplers in Chapter 2, followed by understanding the fundamentals and theory of operation of a grating coupler in Chapter 3. Chapter 4 gives a brief overview of the numerical methods employed for the design and simulation of the grating couplers. The methodology and simulation results of the proposed design are reported in 
Chapter 5, along with the SWGC design for fabrication. Chapter 6 concludes the work and lists the achievements associated with the broadband and polarization flexible SWGC and suggests any further development in the same. 


\section{Chapter 2}

\section{Literature Review}

Grating couplers exhibit polarization sensitivity with low coupling bandwidths. In the last 10 years, substantial amount of work has been reported which addresses either one of the two limitations. Most of the reported broadband grating coupler designs are polarization sensitive and support only one polarization, whereas the polarization independent designs have a small coupling bandwidth. In this chapter, we first review and discuss the literature targeted towards improvement of the operating bandwidth of the grating couplers. We move on to discuss the grating coupler designs which exhibit polarization independence. We also include validity checks in this chapter for two of the reported grating couplers.

\subsection{Broadband Grating Couplers}

The broadband grating coupler designs reported in literature utilize the refractive index engineering property of sub-wavelength structures to reduce the average grating index which results in an increased coupling bandwidth. One such grating design proposed and fabricated by Wang et al.([11]) is illustrated in Figure 2.1. They used 1-D Sub-Wavelength Gratings (SWG) for coupling of TE polarized guided mode. The low and high index regions are denoted by $\Lambda_{\mathrm{L}}$ and $\Lambda_{\mathrm{H}}$ respectively in Figure 2.1. The high and the low index region are made up of sub-wavelength structures which allow for precise engineering of the average effective index of the 
grating. The high index regions have a larger fill-factor/ duty-cycle resulting in wider unetched sub-wavelength silicon structures. Similarly, the low index region has lower fill-factors which results in reduced refractive index.

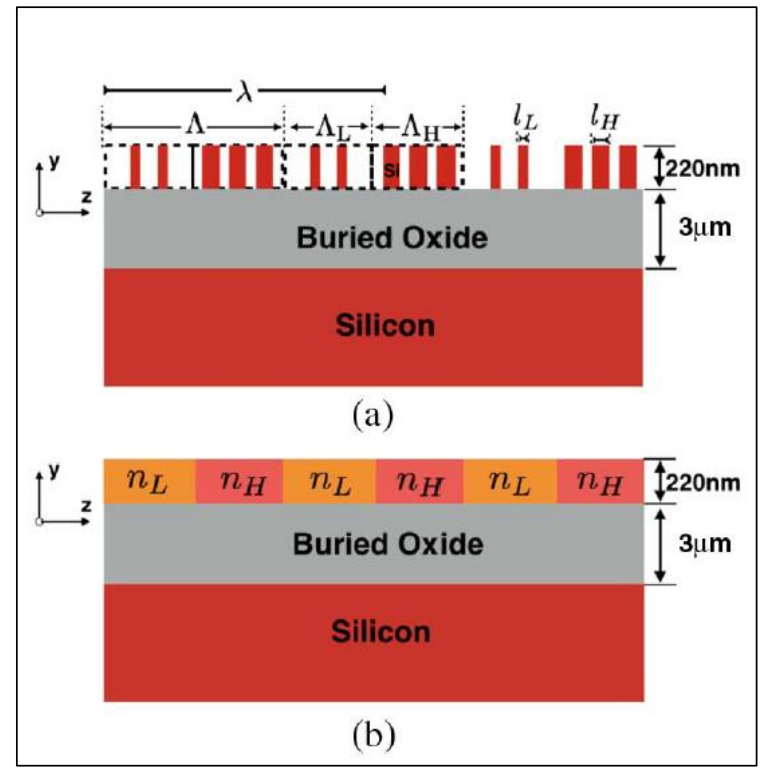

Figure 2.1. Schematic of the cross section of the SWGC with one-dimensional subwavelength gratings; (b) schematic of and SWGC with refractive index regions of $\mathrm{n}_{\mathrm{H}}$ and $\mathrm{n}_{\mathrm{L}}$ (Wang et al.)

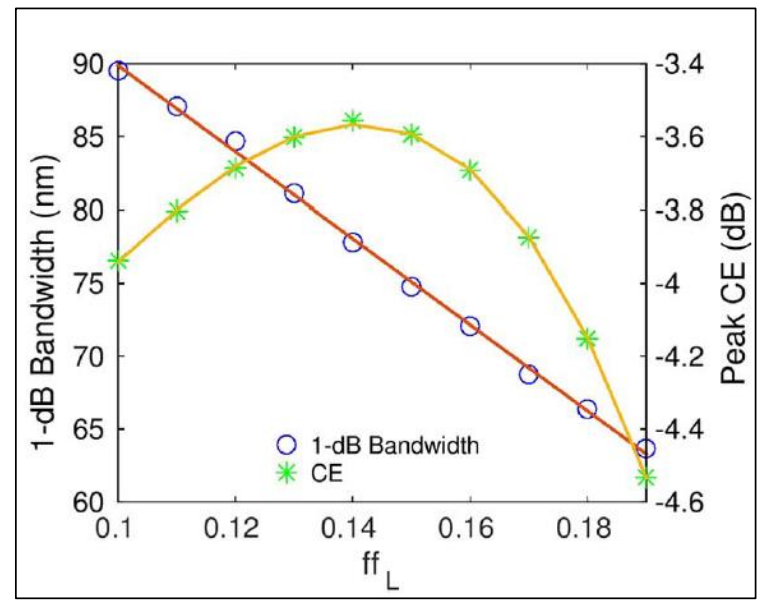

Figure 2.2. Simulated 1-dB bandwidth and peak $\mathrm{CE}$ as a function of $\mathrm{ff}_{\mathrm{L}}$ for the SWGC designed by Wang et al. 
The coupling efficiency and bandwidth results reported by Wang et al. for varying fill-factor of the low index region are depicted in Figure 2.2. It can be observed from the graph that, the maximum coupling efficiency of $-3.6 \mathrm{~dB}$ was observed at $\mathrm{ff}_{\mathrm{L}}=0.14$, with a $1-\mathrm{dB}$ bandwidth of $84 \mathrm{~nm}$ for the SWGC based design. They also reported a measured 1-dB bandwidth of $90 \mathrm{~nm}$ using a focusing grating and a coupling efficiency of $-5.5 \mathrm{~dB}$ at central wavelength of $1578 \mathrm{~nm}$ for SWGC with $\mathrm{ff}_{\mathrm{L}}=0.1$ and $\mathrm{ff}_{\mathrm{H}}=0.52$. The SEM image of the fabricated device is shown in Figure 2.3.

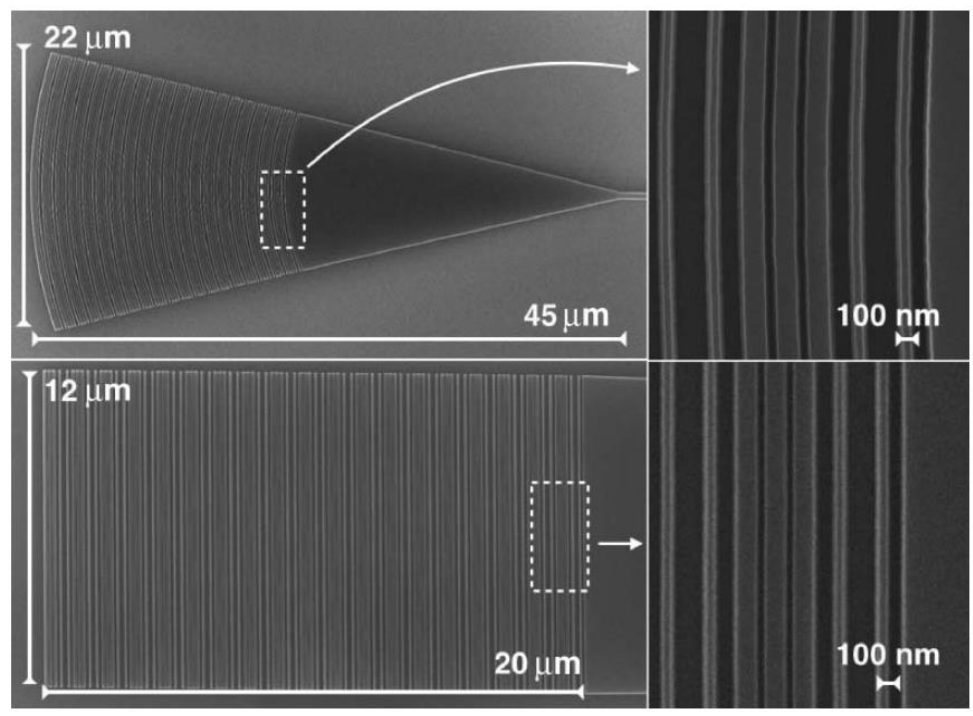

Figure 2.3. SEM images of the as-fabricated focusing and straight SWGC for design reported in Ref. [11]

Another grating design with broadband operation was proposed by Zhong et al. in [12]. They reported the design and characterization of a focusing-curved 2-D SWGC where 1dB-bandwidth of $\sim 100 \mathrm{~nm}$ and a maximum $\mathrm{CE}$ of $-4.7 \mathrm{~dB}$ for TE polarization were achieved (Figure 2.7). They used anti-reflective structures to reduce 
back reflections by tapering the connections between the high and low refractive index regions. The device geometry is illustrated in Figure 2.4. As observed from Figure 2.5, the simulated 1-dB coupling bandwidth for TE polarization is greater than $100 \mathrm{~nm}$ with maximum coupling efficiency approximately $-2.5 \mathrm{~dB}$. The SEM image of the focusing-curved grating coupler is shown in Figure 2.6.

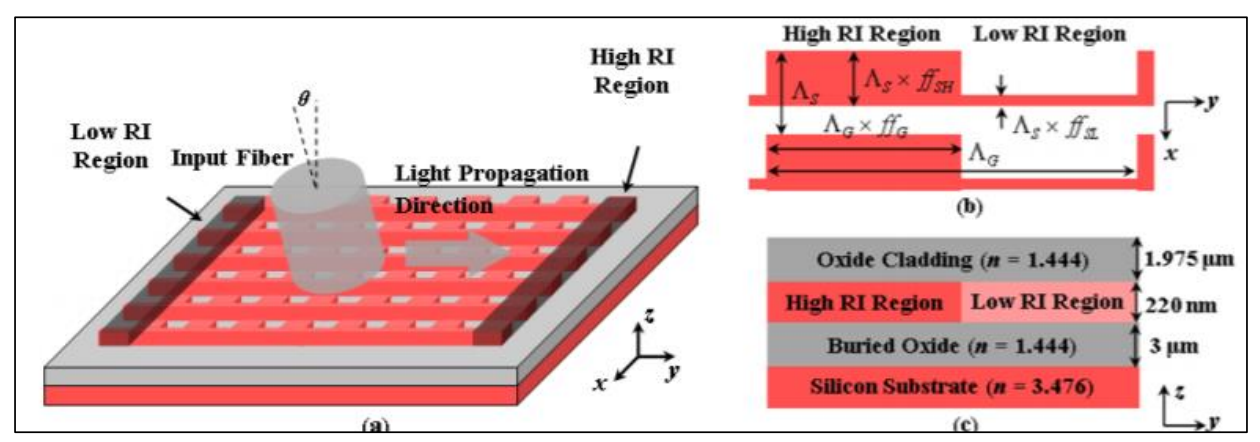

Figure 2.4. Schematic Illustration of the broadband grating coupler design proposed in [12]

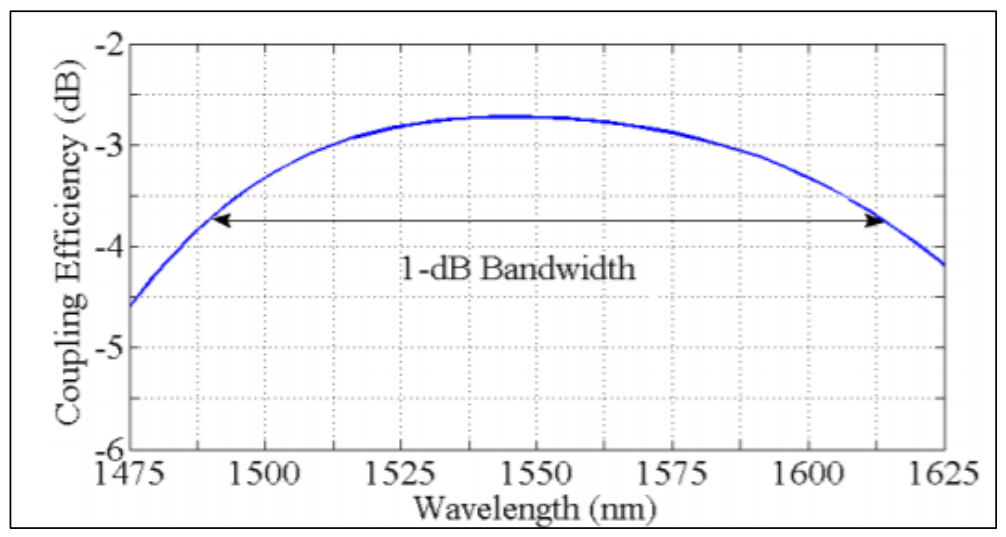

Figure 2.5. Transmission spectrum for the simulated device in [12] 


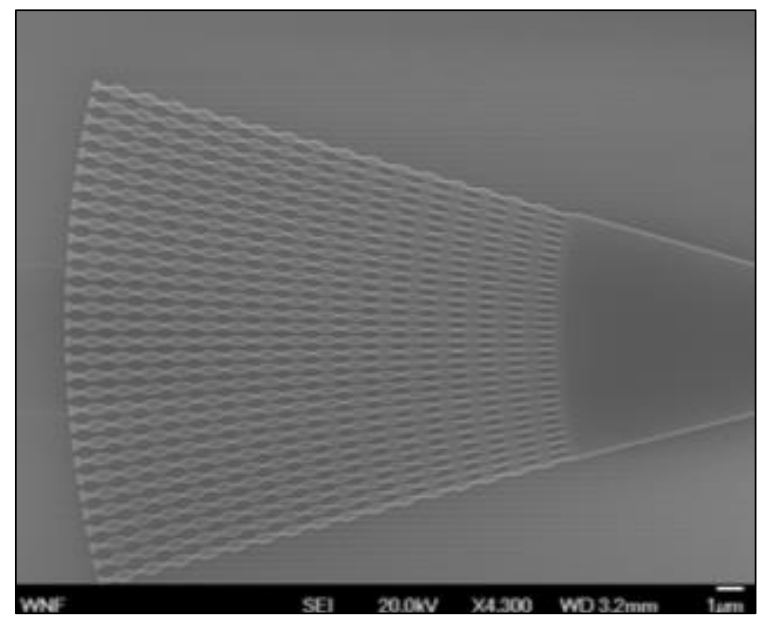

Figure 2.6. SEM image of the fabricated focusing-curved SWGC fabricated and demonstrated in [12]

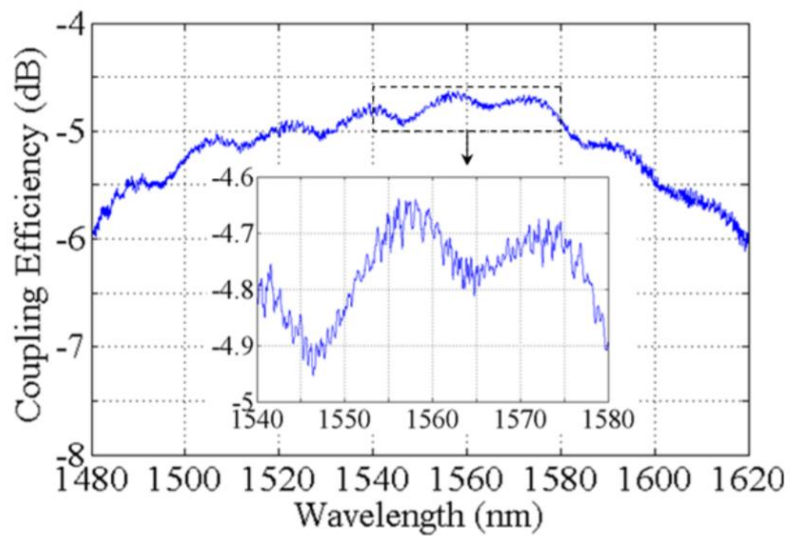

Figure 2.7. Measured transmission spectrum of the focusing-curved SWGC tapered grating design (Ref. [12])

\section{Validity Check}

We selected the 1D- broadband SWGC designed by Wang et al. for validity check.

We used 2D-FDTD simulation to reproduce the design performance with the same software as used by them, Lumerical@ FDTD Solutions. The simulated transmission 
spectrum as attained by them is shown in Figure 2.8 and the one obtained by us for the same grating parameters is shown in Figure 2.9.

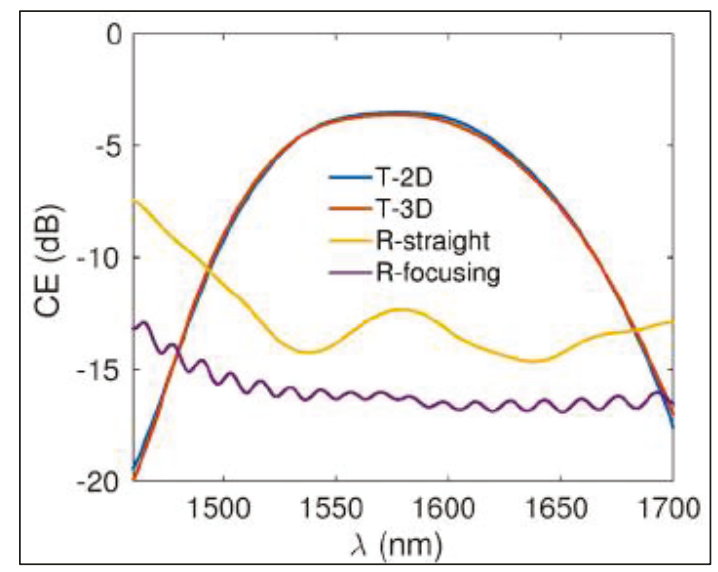

Figure 2.8. Simulated transmission and reflection spectra of the simulated device in [11]

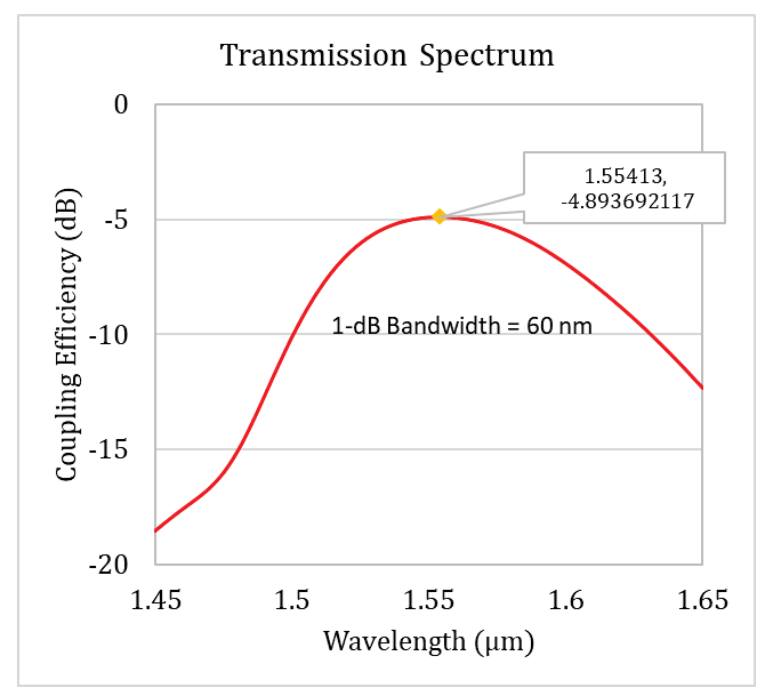

Figure 2.9. Transmission spectrum of the design reported in [11] reproduced using Lumerical FDTD Solutions for straight waveguide

On comparing the transmission spectrum reported in [11] and the one reproduced by us the following were observed- (1) for the angle of incidence reported in the literature, $25^{\circ}$, the grating coupler demonstrated a maximum transmission of 
only $-6.48 \mathrm{~dB}$ with a very narrow $1-\mathrm{dB}$ bandwidth of $27.58 \mathrm{~nm}$, whereas as per the literature a coupling efficiency of $-3.6 \mathrm{~dB}$ with $1-\mathrm{dB}$ bandwidth of $>84 \mathrm{~nm}$ was attained by them; (2) Since, the initial simulation did not give similar results, the farfield angle was then calculated by changing the operating mode of the coupler to outcoupling and was found to be $19.1^{\circ}$; (3) when the TE polarized light was launched at an angle of $19.1^{\circ}$, the maximum coupling efficiency of $-4.89 \mathrm{~dB}$ was observed with a 1-dB coupling bandwidth of $60 \mathrm{~nm}$. In summary, the reproduction of the results as reported in [11] was unsuccessful for the same device design and the validity of the design could not be asserted. However, the results reproduced by us in Figure 2.9 are best comparable to another result reported by Yang et al. in the same paper for $\mathrm{ff}_{\mathrm{L}}=0.20$ as shown in Figure 2.2. This difference could be attributed to the difference in the software versions used or variation in the simulation conditions. A new PML boundary condition formulation was introduced in the 2015 release of the Lumerical FDTD solvers, which may have resulted in the divergent results. Apart from the software-based discrepancy, the background conditions for the simulations are not implicit in the paper which may also cause difference in the two results and hence a failed in validity check.

\subsection{Polarization Independent Grating Couplers}

In this section, we draw our attention towards the design of polarization independent grating couplers. A substantial amount of work has been carried out, which concentrates on the design of polarization insensitive grating couplers. Most of 
the polarization independent designs reported in the literature exhibit small coupling bandwidths.

Zhang et al. [13] designed and analyzed a 1D-GC for coupling the incident $\mathrm{TE}_{0}$ and $\mathrm{TM}_{1}$ polarizations into the planar silicon waveguide. The $\mathrm{TM}_{1}$ polarization that was coupled into the silicon waveguide, was then converted into $\mathbf{T M}_{0}$. At 1550 $\mathrm{nm}$, they reported a maximum $\mathrm{CE}$ of $60 \%$ for both TE and TM, with a limited 1-dB bandwidth of $30 \mathrm{~nm}$ for $\mathrm{TE}_{0}$ and $40 \mathrm{~nm}$ for $\mathrm{TM}_{1}$. This design also required an additional structure for the conversion of $\mathrm{TM}_{1}$ to $\mathrm{TM}_{0}$.

Another polarization splitter grating based on partially etched silicon was proposed by Tang and Dai [14] which obtained a coupling efficiency of approximately $3.00 \mathrm{~dB}$ CE for both TE and TM polarization at $1550 \mathrm{~nm}$ with a simulated $3 \mathrm{~dB}$ bandwidth of $70 \mathrm{~nm}$. The schematic diagram of this polarization splitting grating coupler is depicted in Figure 2.10.

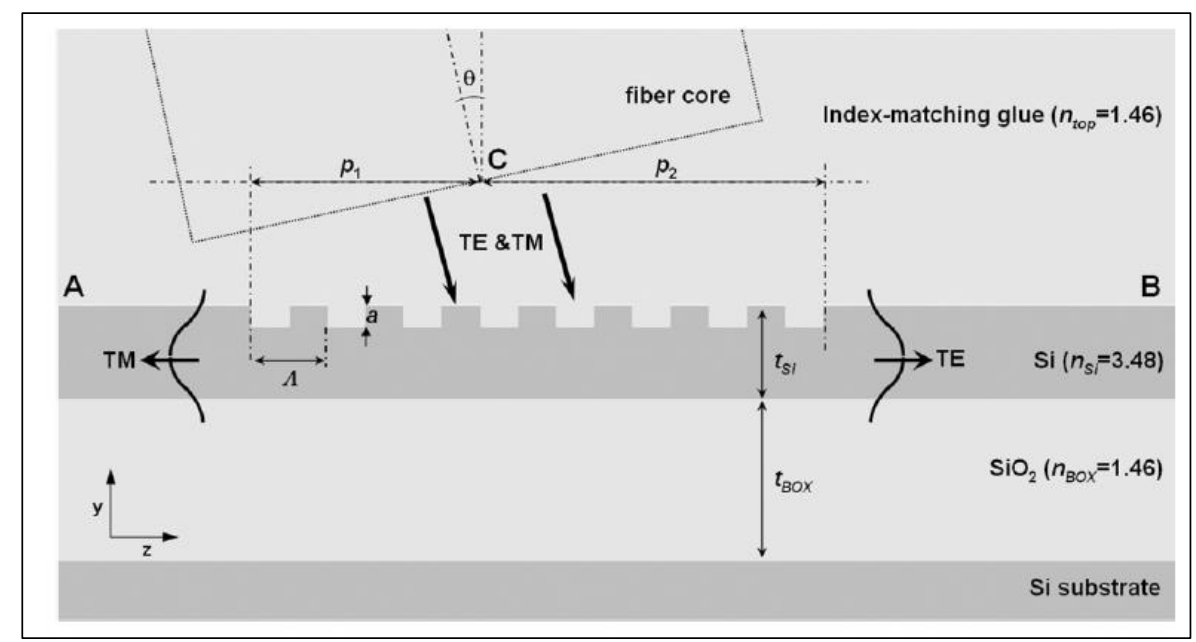

Figure 2.10.Proposed structure by Tang and Dai served as both a polarization splitter and a vertical [14]. 
Polarization diverse grating couplers have also been designed using apodized focusing SWGs. The grating coupler fabricated by Zhenzhou and Tsang [15] has a suspended SWGC to couple both the polarizations into the same waveguide. The BOX layer below the GC was required to be etched away to increase the refractive index contrast, introducing further complexity to fabrication. A maximum experimental $\mathrm{CE}$ of $-3.2 \mathrm{~dB}$ with a $1-\mathrm{dB}$ bandwidth of $28 \mathrm{~nm}$ for the TM polarization was reported, while a maximum $\mathrm{CE}$ of $-4.3 \mathrm{~dB}$ and $1 \mathrm{~dB}$ bandwidth of $58 \mathrm{~nm}$ were demonstrated for TE. The coupling peaks for the two polarizations were not coincident but, differed by a minimum of $\sim 32 \mathrm{~nm}$. The also group claimed a polarization insensitive behavior at $1525 \mathrm{~nm}$, where the two-transmission spectrum intersect with a $\mathrm{CE}$ of $-6.5 \mathrm{~dB}$, as depicted in Figure 2.14. The proposed device structure, the fabricated device and the measured simulated performance characteristics are illustrated in the Figure 2.11- Figure 2.14 below.

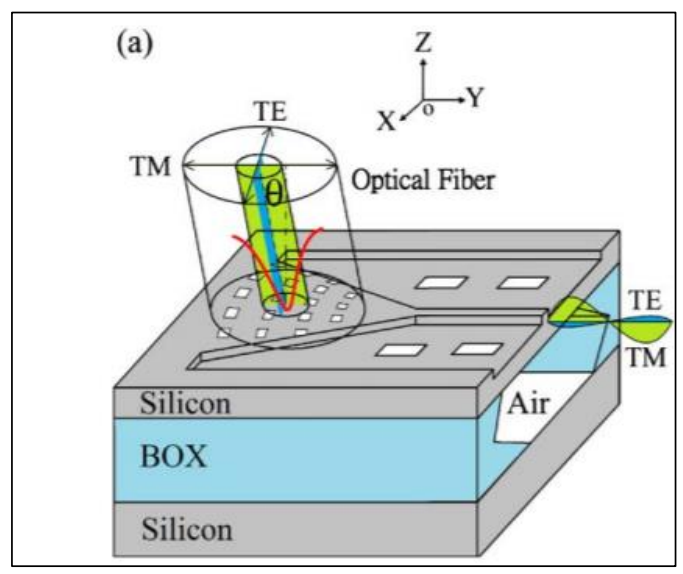

Figure 2.11. Schematic of an apodized focusing SWG for coupling two polarization into a single SMW (Ref. [15]) 


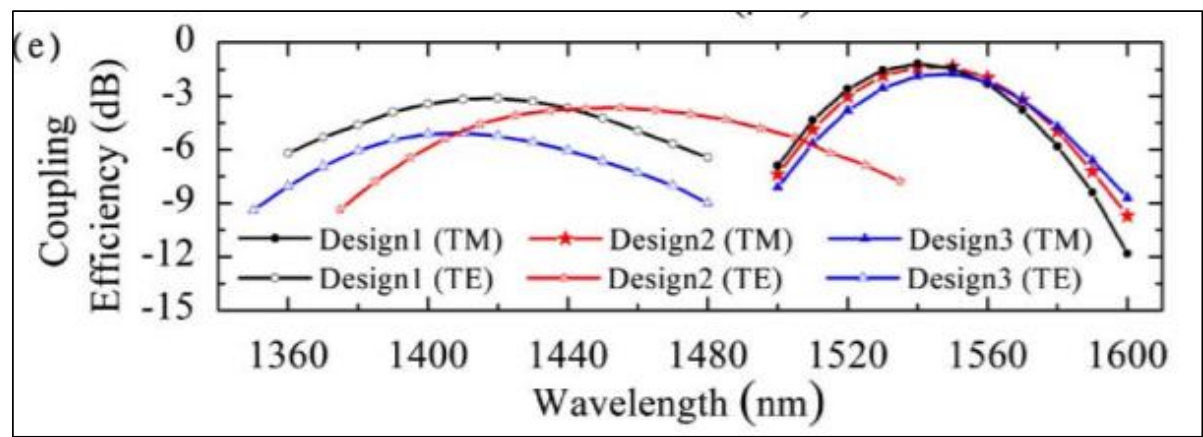

Figure 2.12. Coupling efficiency of the apodized SWG designs using 2D FDTD simulation (Ref. [15])

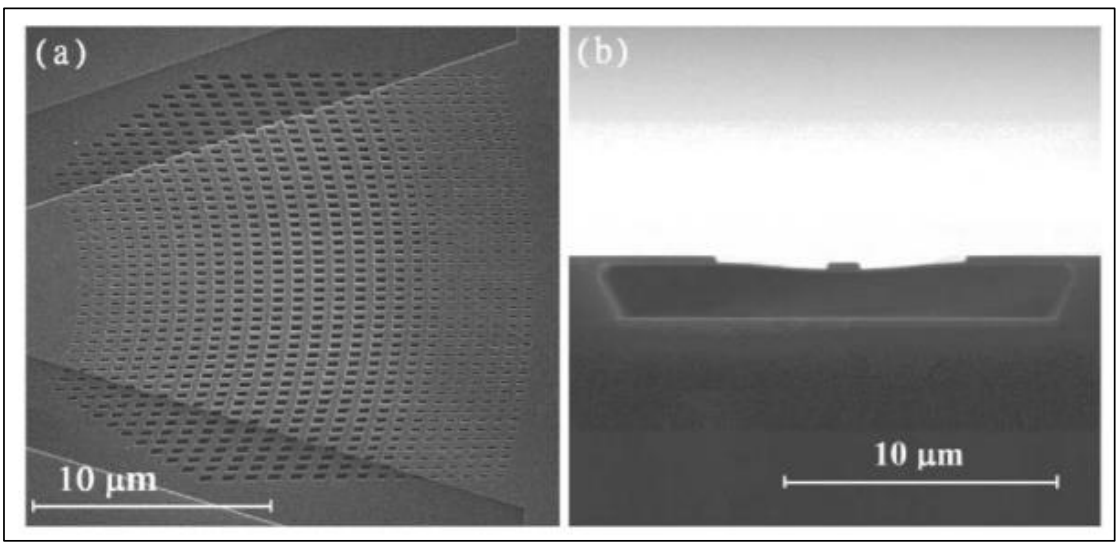

Figure 2.13 (a) Scanning electron microscope (SEM) image of the apodized focusing SWGs. (b) SEM image of the cross section of a SMW (Ref. [15]) 


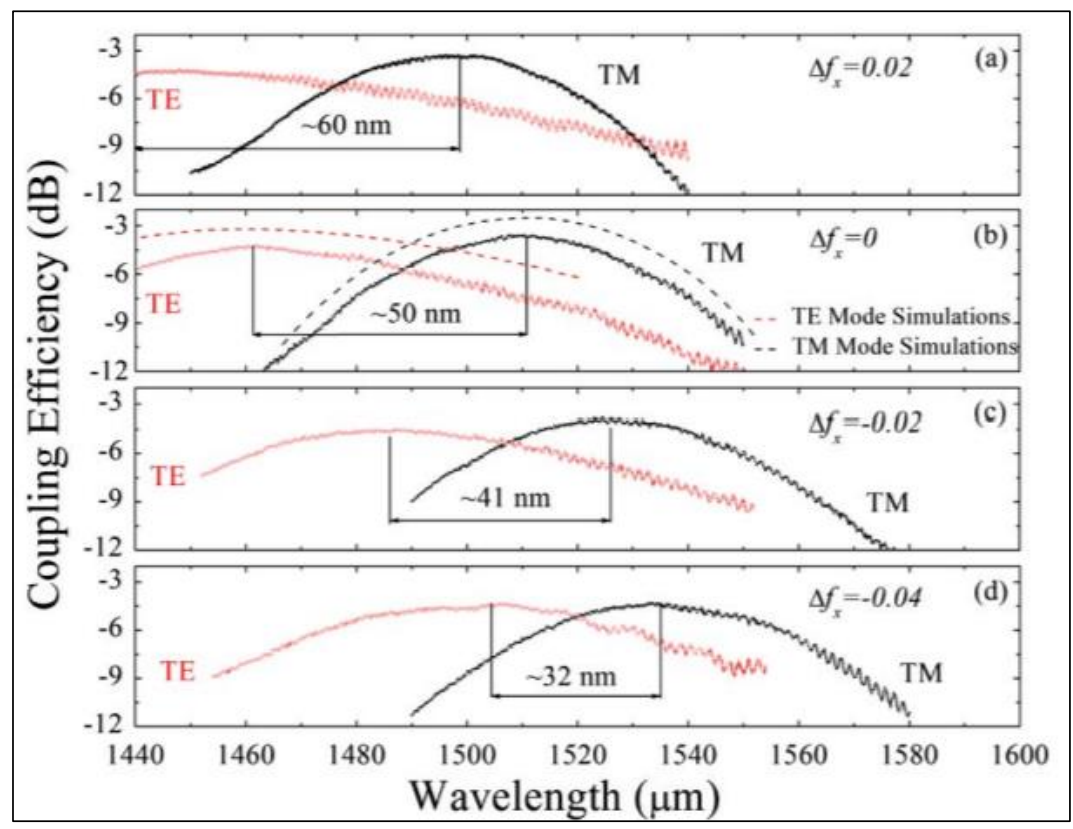

Figure 2.14. Measurements of apodized focusing SWGs with different fill-factors of the sub-wavelength structure designed in [15]

A novel polarization independent non-uniform grating coupler was proposed by Song et al. in 2015, where the gratings are composed of intersection or union of a set of two near-optimal TE- and TM-grating periods, shown in Figure 2.15.

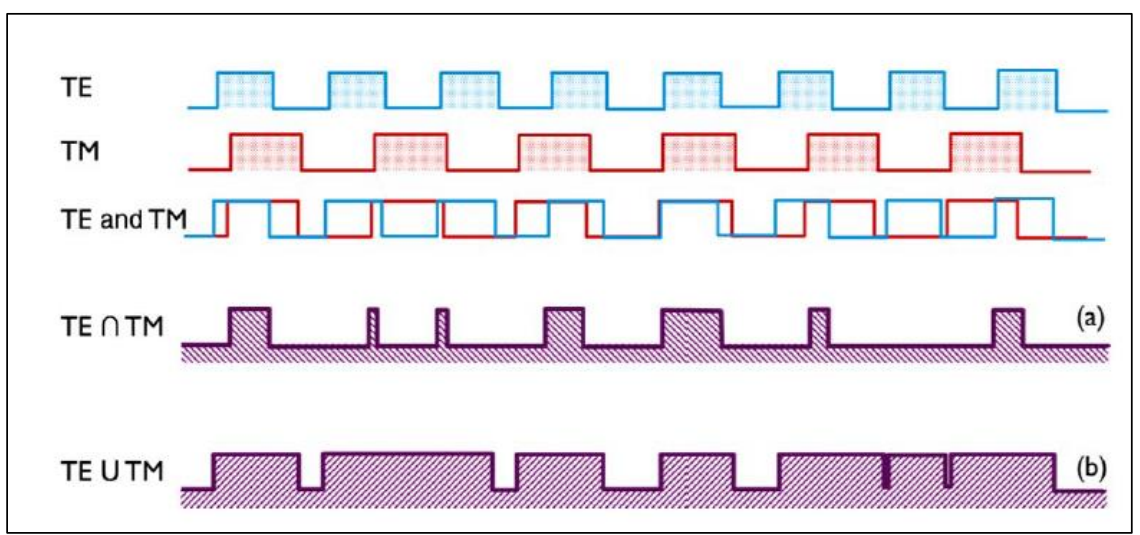

Figure 2.15. Schematic of the nonuniform grating period formation (a) intersection and (b) union of TE and TM mode grating periods. (Ref. [35] ) 
The design was based on a commercial SOI wafer with $t_{S i}=220 \mathrm{~nm}$ and $2 \mu \mathrm{m}$ deep buried oxide. Their intersection and union grating couplers achieved simulated coupling efficiencies of $-6.9 \mathrm{~dB}$ and $-5.4 \mathrm{~dB}$ at the wavelength of $1.55 \mu \mathrm{m}$, respectively. The reported $1-\mathrm{dB}$ bandwidth of both of the grating couplers was approximately $40 \mathrm{~nm}$. For the intersection design, the TE and TM peak coupling efficiencies were $-6.9 \mathrm{~dB}$ and $-7.1 \mathrm{~dB}$ near $1550 \mathrm{~nm}$, respectively. The transmission spectrum for both the grating coupler variants are shown in Figure 2.16 below. The transmission spectrum for the intersection grating is depicted in Figure 2.16 (a) with a maximum $\mathrm{CE}$ of $\mathrm{TE}$ and $\mathrm{TM}$ both $\sim-7.0 \mathrm{~dB}$, with maximum polarization dependent losses of about $0.6 \mathrm{~dB}$ at $1580 \mathrm{~nm}$. A similar transmission spectrum for the union of TE and TM gratings is depicted in Figure 2.16 (b), where the maximum CE of TE is greater than TM and bandwidth of TM is greater than that of TE. This non-uniform 'union' grating exhibits maximum PDL of almost 1-dB at $1590 \mathrm{~nm}$. 


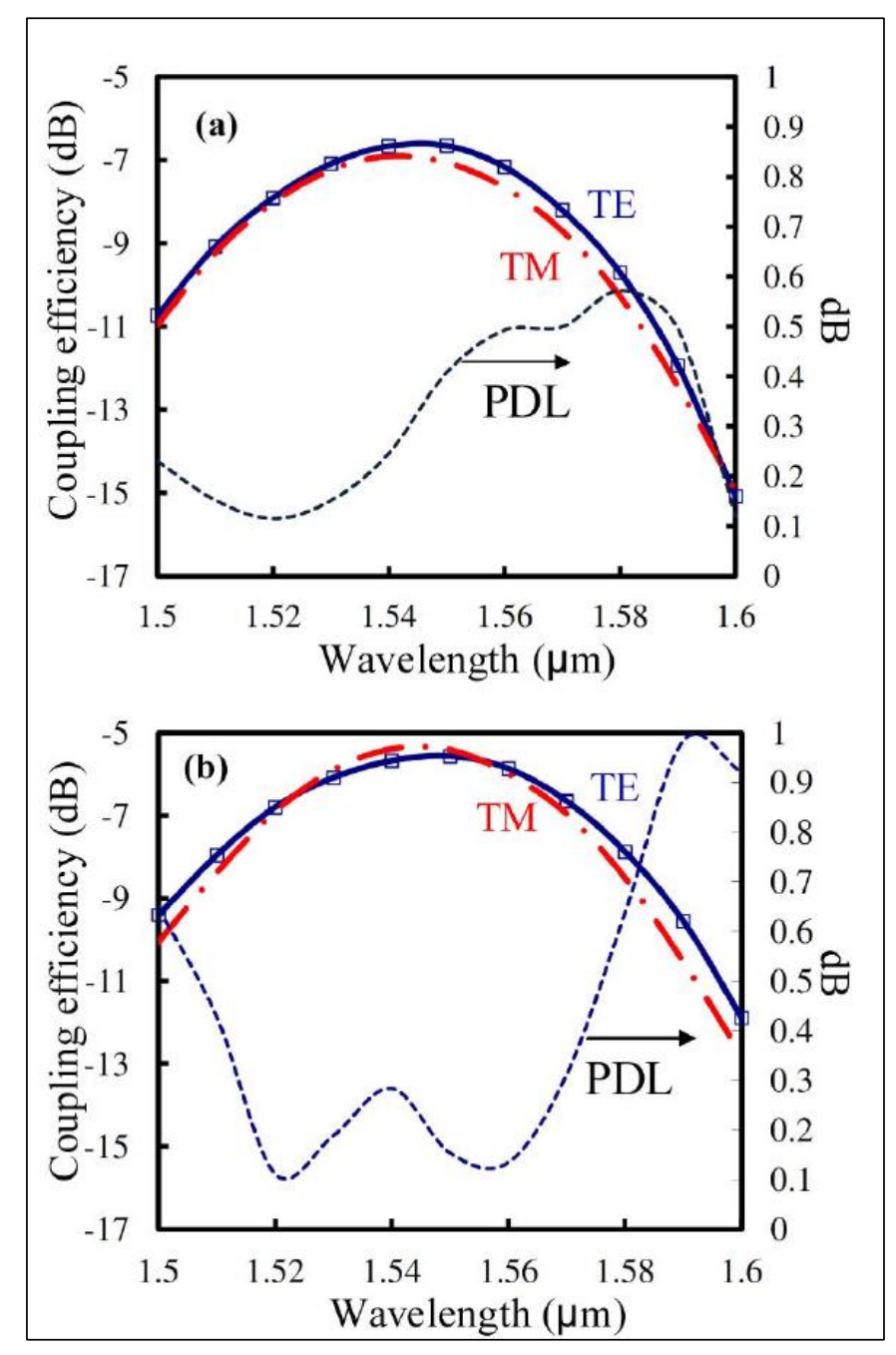

Figure 2.16. Fiber coupling performance of optimized (a) intersection and (b) union grating couplers (Ref. [35])

\section{Validity Check}

We conduct a validity check for polarization splitting design proposed by Tang and Dai. The transmission spectrum reported by them is depicted in Figure 2.17 and the transmission spectrum obtained by us is plotted in Figure 2.18. It is evident from the two graphs that both the transmissions spectrum are equivalent with little variations 
indicating that we were successful in reproducing the results using Lumerical ${ }^{\circledR}$ FDTD Solutions.

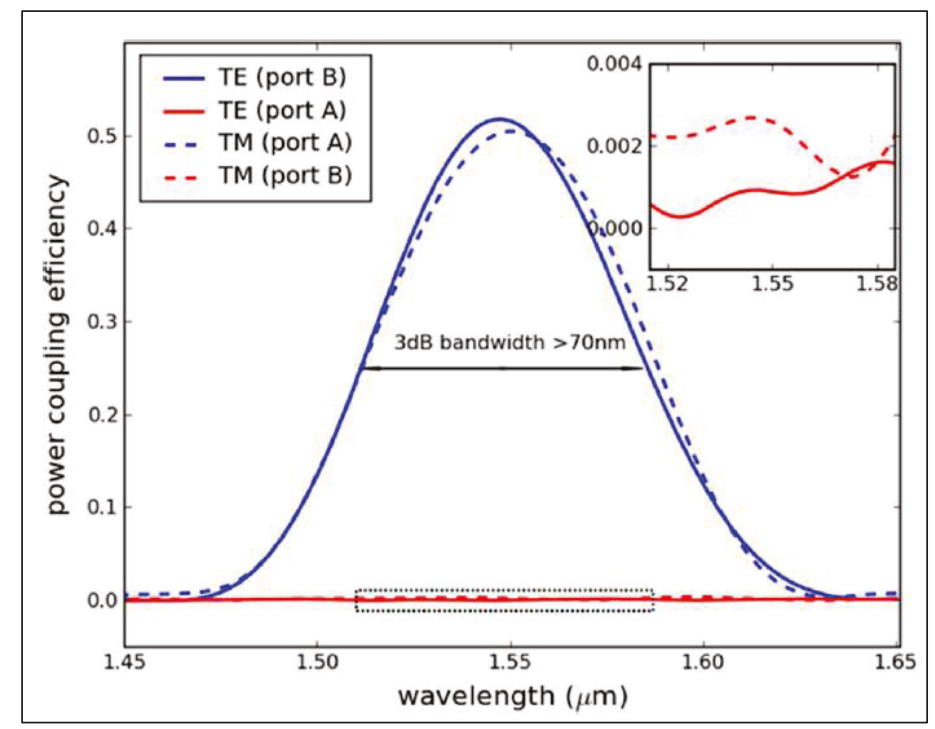

Figure 2.17. Wavelength dependence of the coupling efficiency for the optimized device ( Ref. [14])

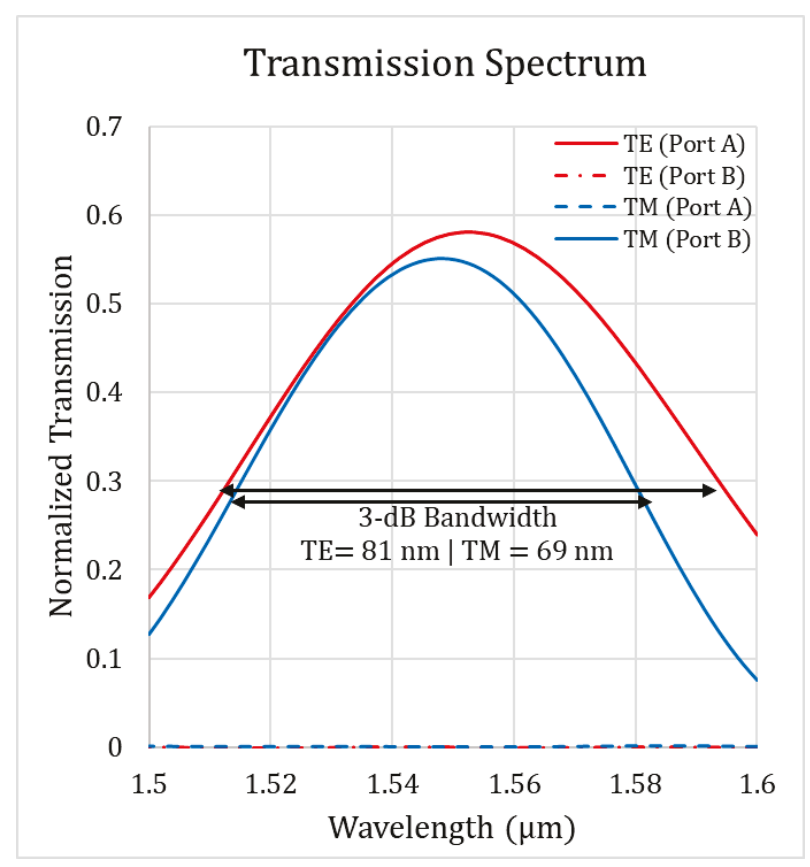

Figure 2.18. Re-generated results from [14], using Lumerical FDTD 
These slight variations could be explained by the difference in the material properties used for simulation. The simulation software itself could also result in the minor discrepancies in the result. Also, since the simulation software's evolve and update with time, it is very likely that the simulation results differ for the same design when simulated with different versions of the same software tool.

\section{Summary}

A summary of the broadband and polarization independent grating couplers is listed in Table 2-1. From the literature review we observed that a grating coupler that is both broadband and polarization independent has not been proposed yet. To start with we conducted validated checks on two of the results. Though, we successfully validated the polarization independent design by Tang and Dai, but the results attained by us for the broadband grating coupler designed by Wang et al. deviated by a large margin from the published results. For this thesis, our objective is to address the small bandwidth and polarization selectivity of the grating coupler. We aim to design a grating coupler with a 3-dB coupling bandwidth of approximately $100 \mathrm{~nm}$ at the central operating wavelength of $1550 \mathrm{~nm}$ which efficiently couples both TE and TM polarization. 
Table 2-1. State-of-the-art grating couplers

\begin{tabular}{|c|c|c|c|c|c|c|c|}
\hline Ref. & Polarization & Maximum Coupling & efficiency & 1-dB Band & th (nm) & 3-dB Bandwidth & Process \\
\hline & & Simulated & Measured & Simulated & Measured & Simulated & \\
\hline $\begin{array}{l}2015, \\
\text { Wang et al. } \\
\text { [11] }\end{array}$ & $\mathrm{TE}$ & $-3.6 \mathrm{~dB}$ & $\begin{array}{l}-5.5 \mathrm{~dB} @ \\
1578 \mathrm{~nm}\end{array}$ & 84 & 90 & Not given & $\begin{array}{l}220 \mathrm{~nm} \mathrm{Si} \text { and } 3 \mu \mathrm{m} \\
\text { BOX, full etch }\end{array}$ \\
\hline $\begin{array}{l}2014, \\
\text { Zhong et } \\
\text { al. [12] }\end{array}$ & $\mathrm{TE}$ & $-2.72 \mathrm{~dB}$ & $-4.7 \mathrm{~dB}$ & 122 & $>100$ & Not given & $\begin{array}{l}220 \mathrm{~nm} \text { Si on } 3-\mu \mathrm{m} \\
\text { BOX and } 1.975-\mu \mathrm{m} \\
\text { oxide cladding, full } \\
\text { etch }\end{array}$ \\
\hline $\begin{array}{l}2015, \\
\text { Zhang et al. } \\
\text { [13] }\end{array}$ & TE \& TM & $\begin{array}{l}\text { TE: } 69.69 \% \\
\text { TM: } 75.15 \% \\
\text { Peak difference: } 30 \\
\text { nm }\end{array}$ & Not given & $\begin{array}{l}\text { TE: } 30 \\
\text { TM: } 40\end{array}$ & Not given & Not given & $\begin{array}{l}460 \mathrm{~nm} \text { Si and } 2 \mu \mathrm{m} \\
\text { BOX, partial etch }\end{array}$ \\
\hline $\begin{array}{l}\text { 2009, Tang } \\
\text { and Dai } \\
\text { [14] }\end{array}$ & TE \& TM & $-3.00 \mathrm{~dB}$ & Not given & Not given & Not given & $\begin{array}{l}\text { TE: } 70 \\
\text { TM: } 70\end{array}$ & $\begin{array}{l}260 \mathrm{~nm} \mathrm{Si} \text { and } 1.95 \\
\mu \mathrm{m} \mathrm{BOX} \text {, partial etch }\end{array}$ \\
\hline $\begin{array}{l}2014, \\
\text { Cheng and } \\
\text { Tsang [15] }\end{array}$ & TE \& TM & $\begin{array}{l}\text { TE: }-3.3 \mathrm{~dB} \\
\text { TM: }-2.5 \mathrm{~dB} \\
\text { Coupling peak } \\
\text { difference } \sim 50 \mathrm{~nm}\end{array}$ & $\begin{array}{l}\text { TE: }-4.3 \mathrm{~dB} \\
\text { TM: }-3.2 \mathrm{~dB} \\
\text { Coupling peak } \\
\text { difference } \sim 32 \mathrm{~nm}\end{array}$ & $\begin{array}{l}\text { TE: } 60 \\
\text { TM: } 30\end{array}$ & $\begin{array}{l}\text { TE: } 58 \\
\text { TM: } 28\end{array}$ & Not given & $\begin{array}{l}340 \mathrm{~nm} \mathrm{Si} \text { and } 2 \mu \mathrm{m} \\
\text { BOX }\end{array}$ \\
\hline $\begin{array}{l}\text { 2015, Song } \\
\text { et al. [35] }\end{array}$ & TE \& TM & $\begin{array}{l}\text { Intersection } \\
\text { TE: }-6.9 \mathrm{~dB} \\
\text { TM: }-7.1 \mathrm{~dB} \\
\text { Union: }-5.4 \mathrm{~dB}\end{array}$ & $\begin{array}{l}\text { Intersection: } \\
\text { TE: }-7.8 \mathrm{~dB} \\
\text { TM: } 8.0 \mathrm{~dB}\end{array}$ & $\begin{array}{l}\text { TE: } \sim 40 \\
\text { TM: } \sim 40\end{array}$ & Not given & Not given & $\begin{array}{l}220 \mathrm{~nm} \mathrm{Si} \text { and } 2 \mu \mathrm{m} \\
\text { BOX and } 60 \mathrm{~nm} \text { etch }\end{array}$ \\
\hline
\end{tabular}




\section{Chapter 3}

\section{Grating Coupler Fundamentals}

This chapter gives an overview of the fundamental principles and theories of the grating coupler. We first touch upon the Bragg's law for periodic media and Bragg condition for grating coupler followed by a brief discussion on detuned grating couplers. The subsequent section focusses on the figure of merit (FOM) which describe the coupler performance. Section 3.1.5 establishes the theoretical approximation for the coupling bandwidth of the grating coupler. Finally, the two most recent state-of-art techniques- the sub-wavelength and apodized grating, are presented which are followed by a brief section on focusing gratings.

\subsection{Braggs Condition}

\subsubsection{Bragg Law}

When the electromagnetic waves are scattered from the periodic media, they depict kinematic properties that are fundamental to all waves, including electron waves, optical waves and acoustic waves [16]. For an infinite periodic medium, it is assumed that the reflection from these planes resemble reflections from a mirror. For a one-dimensional periodic medium, having periodic index variation of $\Lambda$, the difference in the path of the rays reflected from two adjacent plans is given by Bragg's Law, equation (3.1). 


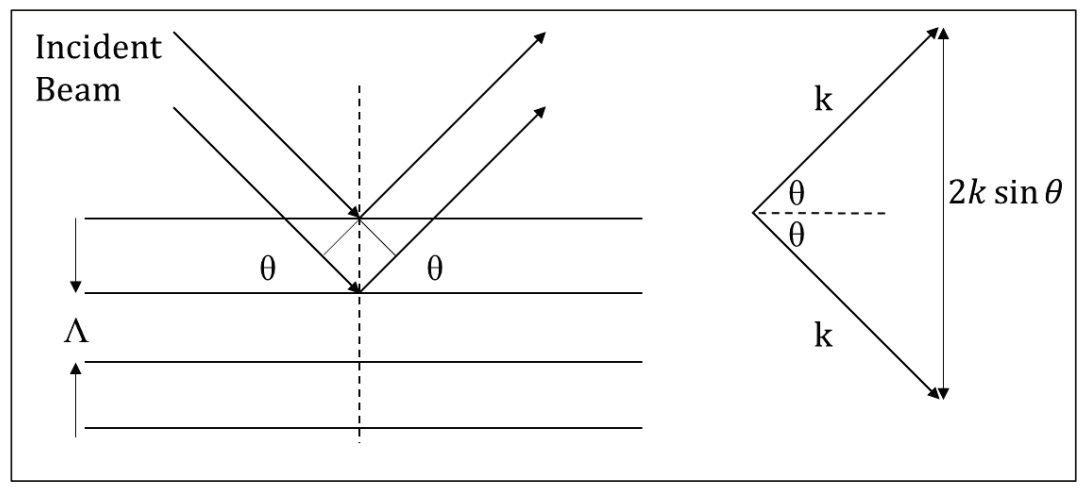

Figure 3.1. Scattering of plane wave from a periodic medium

$$
2 \Lambda \sin \theta=m\left(\frac{\lambda}{n}\right)
$$

where, $\mathrm{m}$ is the order of diffraction (integral value), $\mathrm{n}$ is the average refractive index of the periodic medium. Diffraction of beam from the periodic medium is possible only for certain values of $\theta$, which obey Bragg's Law such that reflections from all the planes add up in phase. Re- writing equation (3.1) :

$$
\begin{gathered}
2 \mathrm{k} \sin \theta=\mathrm{m} \cdot \frac{2 \pi}{\lambda} \\
\mathrm{k}=\frac{2 \pi \mathrm{n}}{\lambda}
\end{gathered}
$$

where, $\mathrm{k}$ is the wavenumber of the light beam in the medium. The term $\frac{2 \pi}{\lambda}$ is the Grating wave-number, given by equation (3.4)-

$$
\mathrm{G}=\frac{2 \pi}{\Lambda}
$$


The Bragg Law complies with the conservation of momentum of the diffracted wave, since the change of wave-vector $\mathrm{k}$ is an integral multiple of the grating wave number $\mathrm{G}$.

\subsubsection{Braggs' Condition for Grating Coupler}

A side view of a partially etched grating coupler is depicted in Figure 3.2 . The periodic nature of the grating causes a periodic modulation of the effective index of the waveguide [21]. This grating consists of alternating regions of partially etched and unetched silicon. The etch depth is given by $e d$. The guided mode propagates perpendicular to the grating teeth, along the direction of the grating period $(\boldsymbol{\Lambda})$. The device shown in Figure 3.2 is based on a $220 \mathrm{~nm}$ thick silicon wafer with a $3 \mu \mathrm{m}$ thick buried oxide (BOX).

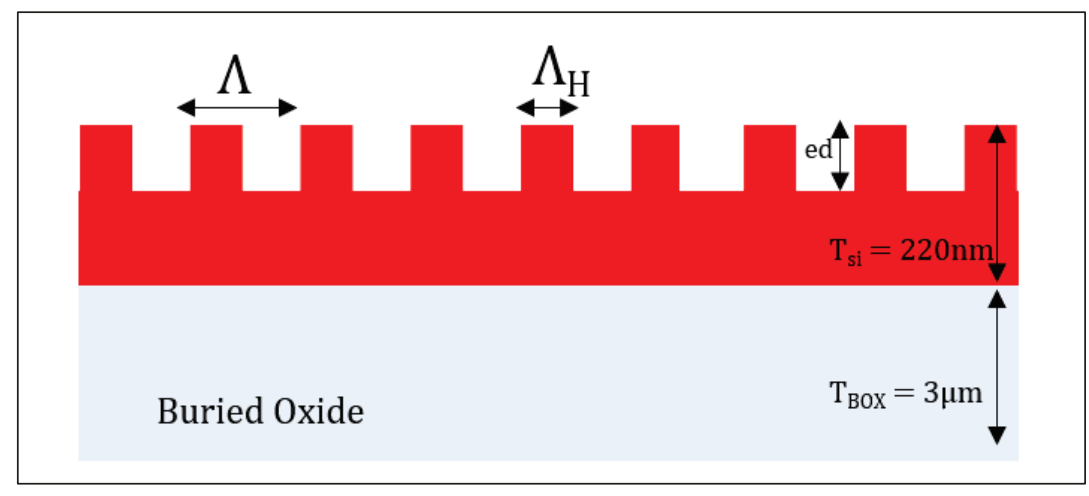

Figure 3.2. Simple grating coupler side view

The duty cycle of the grating, also known as the fill-factor (ff), is defined as

the ratio of the length of the unetched silicon to the grating period, ff $=\frac{\Lambda_{\mathrm{H}}}{\Lambda}$. The Bragg's coupling condition governs the successful and efficient coupling between the guided mode in the planar waveguide and the radiated mode. The device parameters 
of the grating determine probability of coupling between the guided and the radiated mode. The general form of Bragg's condition is given in equation (3.5):

$$
\beta_{\mathrm{o}}-\beta-\mathrm{m} \cdot\left(\frac{2 \pi}{\Lambda}\right)=0
$$

where, $\beta_{\mathrm{o}}$ is the propagation constant of the guided mode and $\beta$ is the propagation constant of the radiated mode. The grating period is $\Lambda$ and $\mathrm{m}$ is the order of diffraction. The magnitude of the propagation constant determines whether the scattered wave shall radiate towards the air/cladding $\left(\mathrm{n}_{\mathrm{c}}\right)$ or the substrate $\left(\mathrm{n}_{\mathrm{SiO}_{2}}\right)$ [16]. Table 3-1 lists the numerous coupling possibilities. Figure 3.3 illustrates the momentum diagram of the forward radiation mode of the diffracted mode into the air.

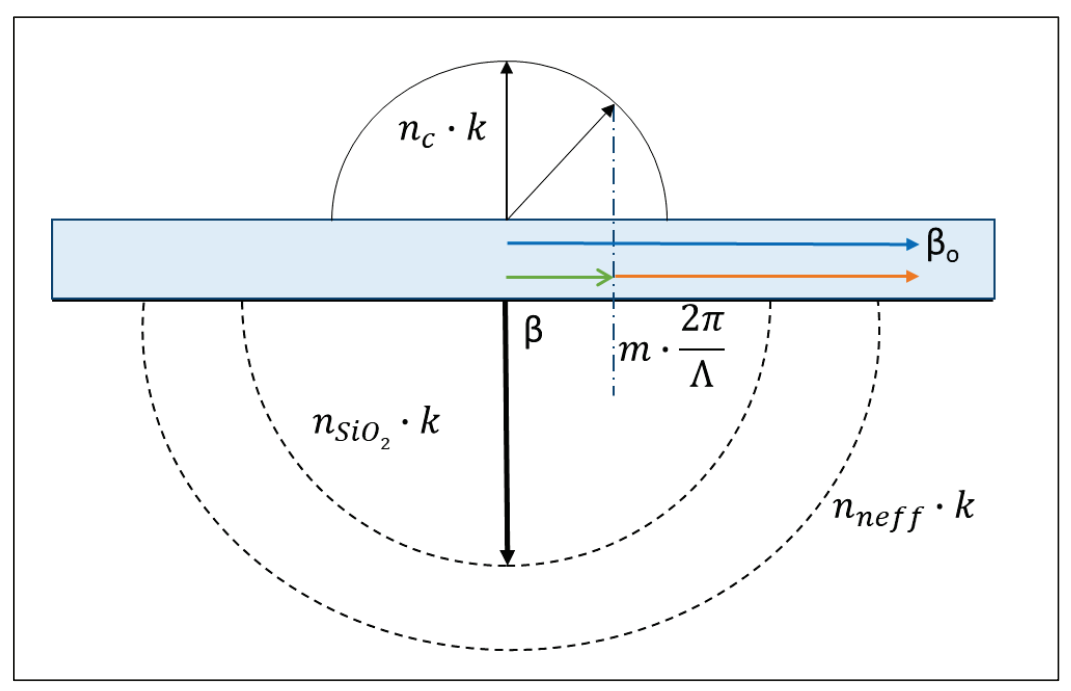

Figure 3.3. Momentum Diagram of Forward Radiation mode into the air/cladding (Ref. [16]) 
Table 3-1. Dependence of $\beta$ on the grating period (Ref. [16])

\begin{tabular}{|r|c|l|}
\hline & \multicolumn{1}{|c|}{$\boldsymbol{\beta}$} & \multicolumn{1}{c|}{ Radiation mode } \\
\hline 1. & $\mathrm{n}_{\mathrm{c}} \mathrm{k}<\beta<\mathrm{n}_{\mathrm{SiO} 2} \mathrm{k}$ & Forward Substrate mode \\
\hline 2. & $0<\beta<\mathrm{n}_{\mathrm{c}} \mathrm{k}$ & Forward Air mode (and substrate mode) \\
\hline 3. & $\beta=0$ & Vertical air mode (and substrate mode) \\
\hline 4. & $-\mathrm{n}_{\mathrm{c}} \mathrm{K}<\beta<0$ & Backward air mode (and substrate mode) \\
\hline 5. & $-\mathrm{n}_{\mathrm{SiO} 2} \mathrm{~K}<\beta<-\mathrm{n}_{\mathrm{c}} \mathrm{K}$ & Backward substrate mode \\
\hline & & \\
\hline
\end{tabular}

$\mathrm{n}_{\mathrm{c}}=$ index of cladding (Air)

$\mathrm{n}_{\mathrm{eff}}=$ index of the guiding layer (Silicon)

$\mathrm{n}_{\mathrm{SiO}_{2}}=$ index of the substrate (Silicon Dioxide)

$\mathrm{K}=\frac{2 \pi}{\Lambda}$ Grating wave-number

The equation (3.5) can be re-written as (3.6), where $\mathrm{k}_{0}$ is the wave-vector of the propagating light wave with wavelength of $\lambda_{\mathrm{o}}$ in free space-

$$
\mathrm{k}_{0} \cdot \mathrm{n}_{\mathrm{eff}}=\mathrm{k}_{0} \cdot \mathrm{n}_{\mathrm{c}} \cdot \sin \theta+\mathrm{m} \cdot \frac{2 \pi}{\Lambda}
$$

$\mathrm{n}_{\text {eff }}$ is the effective index of the propagating mode in the silicon waveguide, $\mathrm{n}_{\mathrm{c}}$ is the index of the cladding, $\theta$ is the angle of incidence of the fiber, $\mathrm{m}$ is the order of 
diffraction, $\mathrm{K}=\frac{2 \pi}{\Lambda}$ and $\Lambda$ is the grating period. High order diffraction gratings are designed with $|m|>1$.

For efficient coupling between the incident mode of the fiber and the guided mode of the planar waveguide, the fiber must be incident at an angle with respect to the surface normal. If the light were to be incident along the surface normal, the reflections experienced at the $\mathrm{Si}_{-} \mathrm{SiO}_{2}$ and at the $\mathrm{SiO}_{2}-$ Substrate interface would result in an equal distribution of the coupled power in two opposite directions, result in a $50 \%$ reduction in the coupled power in any one direction. Apart from this, it is also necessary to couple the light under a small angle to eliminate the first-order Bragg reflection (termed "detuning grating", discussed in the following section); therefore, grating couplers seldom are designed for perfectly vertical operation [8].

\subsubsection{Detuned Bragg Gratings}

Grating couplers in which, the effective wavelength of the guided mode in the planar waveguide is less than the grating period $(\Lambda)$ are referred to as Detuned Gratings. In these gratings, the back reflections are reduced, and the light is diffracted at a small angle. This reduces the second order Bragg reflections. When the effective wavelength in the grating equals the grating period, the first-order diffraction gives rise to vertical radiation and the second order diffractions propagate back into the waveguide, interfering with the propagating mode. In practical applications, the backreflections are undesired because they lead to Fabry-Perot oscillation between the input and output coupler [8]. To avoid the second-order diffraction, the grating period is selected carefully so that effective wavelength is less than the period. 


\subsubsection{Figure of Merit}

To compare between two grating couplers, the grating performance must be quantified through measurable quantities which describes the grating coupler's efficiency as an optical $\mathrm{I} / \mathrm{O}$ coupler. The FOMs that entirely describe the grating coupler performance are-

1. Coupling Efficiency (CE)/ Insertion Loss: It is the power that is successfully coupled from the silicon waveguide into the fiber's fundamental mode and viceversa.

2. Reflection loss into the waveguide: Due to the large difference in the refractive index of $\mathrm{Si}$ and $\mathrm{SiO}_{2} / \mathrm{Air}$, huge reflections are observed at the waveguide- grating interface. These reflections are highly undesired as they tend to oscillate resulting in Fabry-Perot oscillations. These must be suppressed to orders of magnitude $20 \mathrm{~dB}$ for practical applications.

3. 1-dB and 3-dB Coupling bandwidths: It is the range of wavelength for which the coupling efficiency is more than the maximum efficiency by $1-\mathrm{dB}$ and $3-\mathrm{dB}$ respectively.

As compared to edge couplers, grating couplers have lower coupling efficiencies and bandwidth. The lower $\mathrm{CE}$ and bandwidths are attributed to the mode mis-match, power lost to substrate, directionality of the radiated beam and the back reflections. 


\section{Mode Mis-match}

The effective index of silicon at $1.55 \mu \mathrm{m}$ is 3.4777 , whereas the cladding may have an index of 1.45 for $\mathrm{SiO}_{2}$ or 1.00 for air. The huge refractive index contrast between the silicon waveguide and the cladding results in a very high mode confinement factor. The silicon waveguide based on a $220 \mathrm{~nm}$ SOI wafer has a height of $\mathrm{t}_{\mathrm{si}}=220 \mathrm{~nm}$ and a width $=500 \mathrm{~nm}$, hence the propagating mode is confined within a rectangular area of $500 \times 220 \mu \mathrm{m}^{2}$ approx. Whereas, the diameter of core of a standard SMF-28 fiber is $8.2 \mu \mathrm{m}$ with a mode field diameter in orders of $10 \mu \mathrm{m}$. Guided mode in an optical fiber have lower confinement factor owing to the very small difference in the core and cladding index.

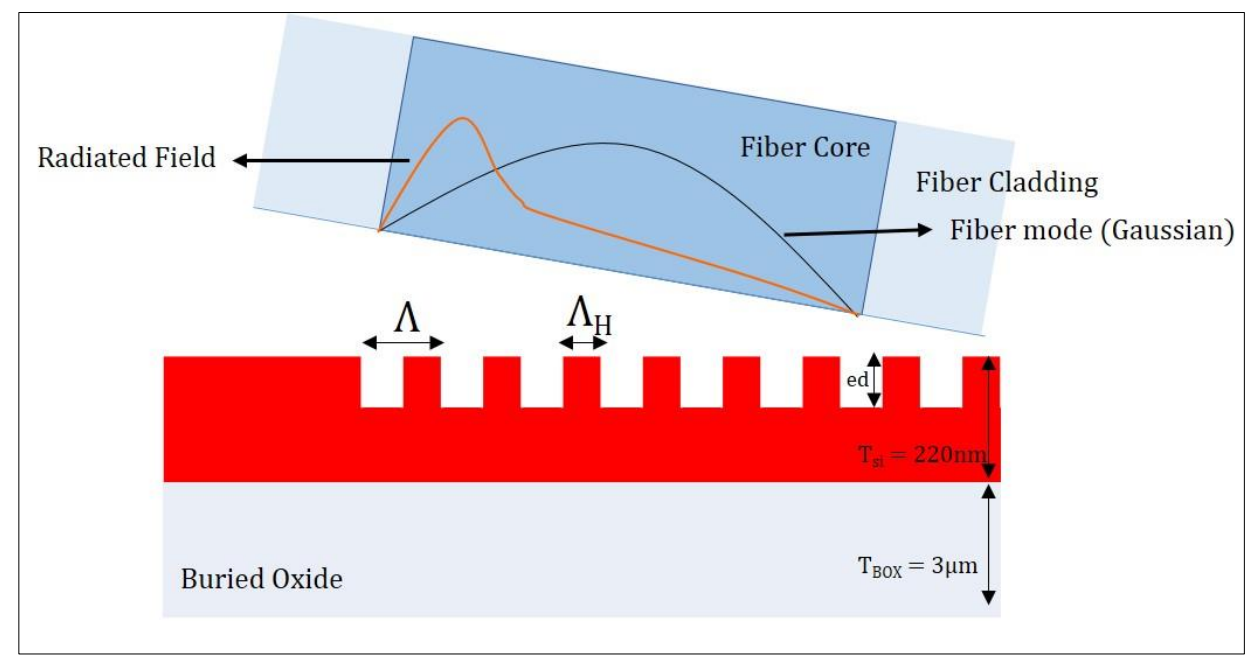

Figure 3.4. Overlap between the radiated beam and the Gaussian fiber mode

Apart from the difference in mode confinement factor, additional power is lost because of the difference in the shape of the radiated beam and the fiber mode. The propagating mode in the fiber has a Gaussian profile, whereas the mode diffracted from the surface grating has an exponential profile. Therefore, reduced overlap 
between the two profiles causes further power loss. The overlap integral between the radiated beam and the near-Gaussian optical fiber mode is given by$\left|\int E_{g} E_{r}^{*} d z^{\prime}\right|^{2}[18]$. The radiated field is calculated by integrating the power that is radiated outwards from all the individual grating period, also known as the strength of the grating. Higher the effective index contrast between the high and the low index regions, stronger is the grating, resulting in a narrow radiated beam with an overlap of about $\sim 0.35$ for SMF-28 fiber mode[20]. For a uniform grating, the radiated beam always resembles an exponential profile. To obtain a radiated field which matches the fiber-mode profile, apodized gratings are used, discussed in Section 3.3.1 . Apodization aids in varying the grating strength along the length so that the radiated beam has a Gaussian profile. The conventional grating structure use shallow etched gratings, to reduce the effective index contrast thus reducing the grating strength and increasing the overlap integral.

\section{Back Reflections}

As discussed above, back reflections are caused when the grating period is equal to the propagating wavelength and due to the difference in the average effective of the waveguide and the grating. Apodization of grating also reduces back-reflections from the grating coupler, since the refractive index discontinuities are smoothed [17]. A deep etch perturbs the refractive index of a given waveguide more than a shallow grating, hence enabling the coupling mechanism, however adding more loss due to increased scattering [21]. Scattering results from the etch because of the increased abruptness of the refractive index discontinuity between each section of the grating 
[21]. These back-reflections propagate through the waveguide system resulting in interference with the propagating mode.

\section{Directionality}

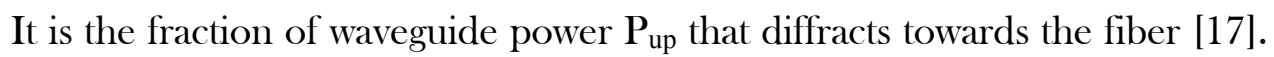
For a given grating, the angle of diffraction of the guided mode is proportional to the propagating wavelength, which results in a different diffraction angles for each wavelength. Thus, not all the radiated power is captured by the optical fiber resulting in lower coupling bandwidths. Theoretical estimation of the coupling bandwidth is discussed in the following section.

\subsubsection{Bandwidth Analysis}

To estimate the bandwidth of the grating, we re-arrange equation (3.6) for $|\mathrm{m}|=1$ and take a differential with respect to theta $(\theta)$ to obtain equation (3.9)-

$$
\begin{gathered}
\frac{\mathrm{n}_{\mathrm{eff}}(\lambda)}{\lambda}=\mathrm{n}_{\mathrm{c}} \cdot \frac{\sin \theta}{\lambda}+\frac{1}{\Lambda} \\
\Delta \lambda_{1 \mathrm{~dB}}=2 \cdot \eta_{1 \mathrm{~dB}}\left|\frac{\mathrm{d} \lambda}{\mathrm{d} \theta}\right| \\
\Delta \lambda_{1 \mathrm{~dB}}=2 \cdot \eta_{1 \mathrm{~dB}} \cdot\left|\frac{\left(-\Lambda \cdot \mathrm{n}_{\mathrm{c}} \cos \theta\right)}{\left(1-\Lambda \cdot \frac{\mathrm{dn}_{\mathrm{eff}}(\lambda)}{\mathrm{d} \lambda}\right)}\right|
\end{gathered}
$$

where $\eta_{1 \mathrm{~dB}}$ is the constant of proportionality and depends on the fiber and grating parameters [19]. $\eta_{1 \mathrm{~dB}}$ is given as 0.07 based on simulation and experimental data [22]. From (3.9), it is evident that the $1-\mathrm{dB}$ bandwidth is directly proportional to the 
grating pitch of the coupler and the dispersion term, $\frac{\mathrm{dn}_{\mathrm{eff}}(\lambda)}{\mathrm{d} \lambda}$. The dispersion term is negative for silicon implying that the effective index reduces with an increase in wavelength. Hence, for grating couplers fabricated in a specific material system, operating at a fixed central wavelength and angle of incidence, the most feasible approach to increase the bandwidth is to reduce the grating effective refractive index [12]. Xiao et al. theoretically investigated the bandwidth behavior of the grating coupler in [19] and observed that the bandwidth is affected by both the grating coupler's intrinsic properties as well as the fiber parameters such as the position, beam waist and numerical aperture. They reported that the 1-dB coefficient, $\eta_{1 \mathrm{~dB}}$ is not a constant value but a function of beam waist, position, and the grating period while the fiber position is optimized for the best efficiency. As per the bandwidth analysis reported in [19], the coupling bandwidth decreases with increase in fiber position as well as the waist radius.

An increase in the coupling bandwidth is noted if the grating is excited by a fiber beam with smaller waist. It was also observed by Xiao et al. that adjusting the beam waist to increase the coupling bandwidth would not induce a great coupling efficiency drop. Since the gaussian beam with a smaller waist has a larger Numerical Aperture, NA $=\frac{\lambda_{0}}{\pi \omega_{0}}$, an increase in NA also increases the coupling bandwidth. Changing the fiber beam waist and the distance changes the effective interaction area, which is described as the grating area effectively covered by the fiber beam, thereby affecting the coupling bandwidth. But for our design, our fiber is assumed to be a standard SMF with a core diameter of $8.2 \mu \mathrm{m}$. 
Hence, for grating couplers operating at a fixed central wavelength, the most feasible approach to increase the optical bandwidth is by reducing the grating effective refractive index [12]. Therefore, both increased dispersion and period will lead to a larger bandwidth[22]. Reducing the GC's effective index can both improve the bandwidth and reduce the coupling efficiency, as the index contrast between cladding and grating is also reduced [12]. Therefore, reducing the average effective index of the grating has dual effects- it increases the coupling bandwidth at an expense of reduction in coupling efficiency. From this, we can infer that the area enclosed by a $\mathrm{CE} \mathrm{v/s} \mathrm{Wavelength} \mathrm{curve} \mathrm{remains} \mathrm{roughly} \mathrm{constant} \mathrm{i.e.} \mathrm{the} \mathrm{average} \mathrm{transmission} \mathrm{is}$ approximately constant.

\subsection{Sub-Wavelength Gratings}

Sub-wavelength structures are employed widely to engineer the refractive index of the grating structure or for silicon waveguides. The structures which have a dimension of the order of $\lambda / 10$ in the direction of propagation of the mode are referred to as sub-wavelength structures. This results in sub-wavelength pitches of the order of $\Lambda \sim 300 \mathrm{~nm}$ for a free-space wavelength $\lambda \sim 1.55 \mu \mathrm{m}$ [17]. Reflection and diffraction effects are suppressed, and the structure operates in the sub-wavelength regime[17]. Light propagates through the SWG without losses despite the multiple discontinuities along the propagation direction[17].

Fully-etched SWGs have the potential to replace shallow-etched gratings. Since the SWGs act as a continuous-homogenous medium, the contrast in the high and the low indices of the grating coupler can be reduced by tuning the resultant 
effective index of the SWGs by varying the fill-factor/ duty-cycle of the subwavelength period. The effective index of a sub-wavelength structure is calculated using Effective Medium Theory (EMT) discussed in Section 4.2

\subsection{Grating Optimizations: Apodized and Focusing Grating}

\subsubsection{Apodization}

To overcome the mode mismatch between the diffracted beam and the optical fiber mode, apodized grating structures are used. Apodization can be achieved by either varying the duty cycle, the etch depth or adding subwavelength structures in the grating [24]. It is already known, that the beam radiated from the grating has an exponential profile, whereas the guided mode of the fiber has a Gaussian profile.

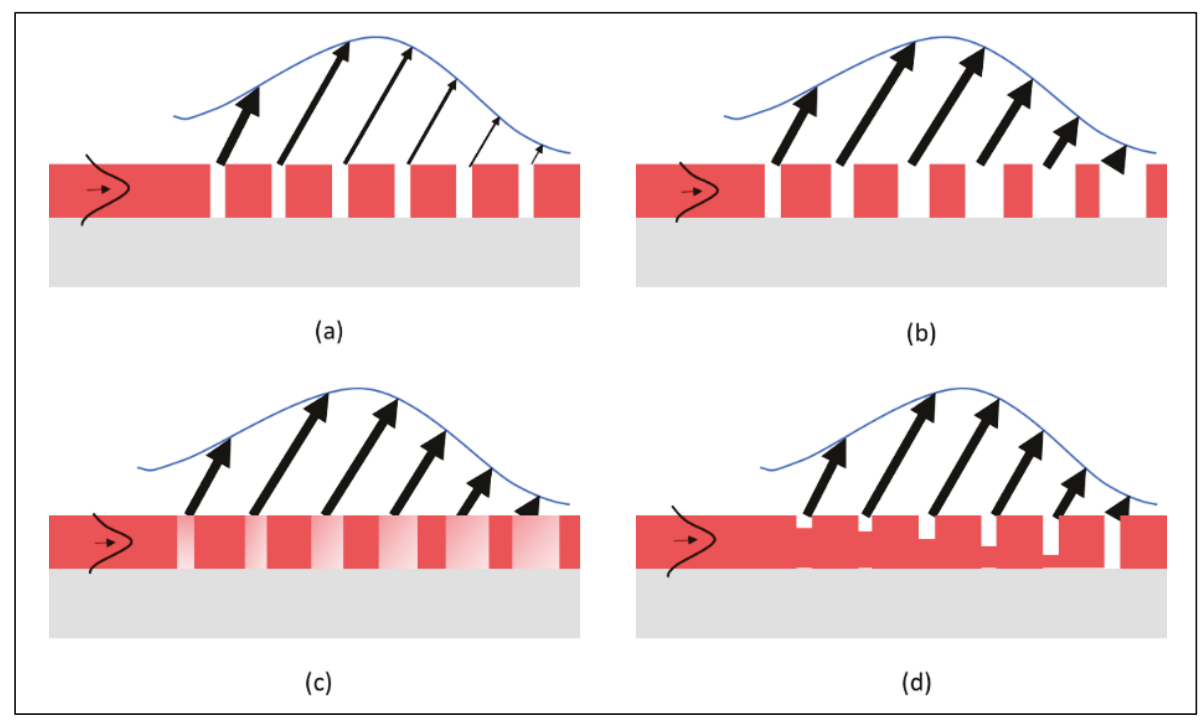

Figure 3.5. Schematic representation of grating couplers a) uniform grating b) based upon duty cycle apodization c) based on SWG apodization c) based on etch-depth apodization (Ref. [24]) 
A uniform grating coupler is depicted in Figure 3.5 (a), where most of the power is radiated from the first couple of grating teeth followed by an exponential decrease in the radiated power from the subsequent grating teeth. In Figure 3.5 (b), the grating has an apodized duty cycle, where the duty-cycle is such that overall the grating strength is lower at the waveguide-grating junction. One can also reduce the grating strength by adding SWG structures in the beginning of the fully-etched trenches, to decrease the effective index contrast, Figure 3.5 (c), thereby reducing the grating strength towards the grating origin. On similar directions etch depth can also be varied along the grating length. As seen in Figure 3.5 (d) etch depth is reduced towards the beginning of the grating to reduce the initial grating strength.

All the above mentioned apodization methods results in a diffracted beam that resembles the Gaussian mode profile of the optical fiber. Using these techniques overlap efficiency of almost $90 \%$ can be achieved.

\subsubsection{Focusing Gratings}

Straight grating couplers are designed to be as wide as the mode field diameter of the single mode fiber approximately $12 \mu \mathrm{m}$. The straight gratings are etched in a $12 \mu \mathrm{m}$ wide waveguide, which are then required to be tapered into a $500 \mathrm{~nm}$ wide nano-wire. This requires an adiabatic taper which may reach up to $500 \mu \mathrm{m}$ in length. Focusing the light, which is diffracted by the grating onto the wire, would obviate the need for this long adiabatic transition, resulting in a substantial decrease in the taper length, and hence a higher degree of integration[26]. 
According to Waldhäusl et al. [27], for a grating located in the y-z plane with the guided mode propagating along the z-direction, the grating lines must follow the condition of constructive interference given below-

$$
q \cdot \lambda_{0}=n_{e f f} \sqrt{y^{2}+z^{2}}-z \cdot n_{c} \cdot \cos \theta_{c}
$$

where $\mathrm{q}$, is the integer for each grating line, $\theta_{c}$, is the angle between the fiber and the chip surface, $n_{c}$ is the refractive index of the environment, $\lambda_{o}$ is the vacuum wavelength, and $n_{\text {eff }}$ is the effective index of the grating. The above equation can be expressed in the polar co-ordinates as well, where $t$ is the variable angle in a polar coordinate system-

$$
\begin{gathered}
x=\sqrt{\frac{q \cdot \lambda_{0} \cdot n_{t} \cdot \cos \theta_{c}+q^{2} \cdot \lambda_{0}^{2}}{n_{e f f}^{2}-n_{t}^{2} \cdot \cos ^{2} \theta_{c}}} \cdot \cos t+\frac{q \cdot \lambda_{0} \cdot n_{t} \cdot \cos \theta_{c}}{n_{e f f}^{2}-n_{t}^{2} \cdot \cos ^{2} \theta_{c}} \\
y=\sqrt{\frac{q^{2} \cdot \lambda_{0}^{2}}{n_{e f f}^{2}-n_{t}^{2} \cdot \cos ^{2} \theta_{c}}} \cdot \sin t
\end{gathered}
$$

This condition in equation (3.10) is only valid under the constraint that the effective refractive index in the grating and the waveguide region is constant [27] i.e. $n_{\text {eff }}^{\text {grating }}=n_{\text {eff }}^{\text {waveguide }}$. For etch-depth greater than $50 \mathrm{~nm}$, the aforementioned condition doesn't hold because the difference in the effective refractive index increase such that-

$$
n_{\text {eff }}^{\text {grating }}-n_{\text {eff }}^{\text {waveguide }}=\Delta n_{\text {eff }} \approx 5 \times 10^{-3}[27] .
$$


Therefore, to account for the difference in the index of the grating and the waveguide, we include an additional correction term in equation (3.10) resulting in equation (3.13) -

$q \cdot \lambda_{0}=n_{e f f}^{g r a t} \sqrt{y^{2}+z^{2}}+z \cdot n_{c} \cdot \cos \theta_{c}+r\left(q_{a}\right)\left(n_{\text {eff }}^{\text {grating }}-n_{\text {eff }}^{\text {waveguide }}\right)$

Where, $r\left(q_{a}\right)$ represents the correction term, which includes the weighted difference of the effective index-

$$
\begin{gathered}
r\left(q_{a}\right)=q_{a} \cdot \lambda \cdot \frac{-z e_{a} \sqrt{y^{2}+z^{2}}+y^{2}+z^{2}}{y^{2}+\left(1-e_{a}^{2}\right) \cdot z^{2}} \\
e_{a}=\frac{n_{c} \cdot \cos \theta_{c}}{n_{e f f}}
\end{gathered}
$$

$e_{a}$, is the numerical eccentricity of the inner ellipse.

The Bragg condition (equation (3.6)) for the curved gratings in polar co-ordinate system is given in equation below [25].

$$
n \cdot k \cdot r=n \cdot k \cdot r \sin \theta \cos \emptyset+2 \pi N
$$

where, $k$, is the wave-vector in free space and $\emptyset$ denotes the angle subtended to the z-axis by an arbitrary point $r$ on the grating. The curvature of the grating does not affect the coupling efficiency. With a given 2D design, the focusing grating coupler can be drawn using the same period and fill-factor. A focusing design of the grating results in reduced area consumption on the SOI wafer along with lower backreflections while giving the same performance as a straight grating coupler.

The next chapter gives an over-view of the numerical methods that were employed for the design and simulation of the grating coupler. 


\section{Chapter 4}

\section{Computational Methods}

This chapter outlines the numerical methods employed for the design of grating coupler. First, the effective index method is presented in Section 4.1 , that estimates the index of refraction of the mode propagating in a two-dimensional silicon waveguide. Section 4.2 focuses on the Effective Medium Theory (EMT). The periodic sub-wavelength structures are perceived as a homogenous medium by the propagating mode and the approximate value of the refractive index of this perceived homogenous medium is obtained using EMT. For simulating the grating structure, we used the Finite Difference Time Domain (FDTD) method which is briefly discussed in Section 4.3 .

\subsection{Effective Index Approximation}

For Silicon-on-Insulator (SOI) wafer, the silicon layer is less than $500 \mathrm{~nm}$ in thickness, while the buried oxide $(\mathrm{BOX})$ can be $2-3 \mu \mathrm{m}$ thick. The silicon is the guiding material or the core, and BOX forms the lower cladding layer. The BOX isolates the guided mode from the silicon substrate and prevents the leakage of the associated fields of the guided optical modes into the substrate [21]. The upper cladding is mostly air $(\mathrm{n}=1.0)$ or $\mathrm{SiO}_{2}(\mathrm{n}=1.5)$. Most applications require the guided mode to be confined in two-dimensions. This is achieved by etching SOI which 
results in a two-dimensional waveguide. The two most common waveguide structures are- the rib structure and the strip waveguide structure, as shown in Figure 4.1.

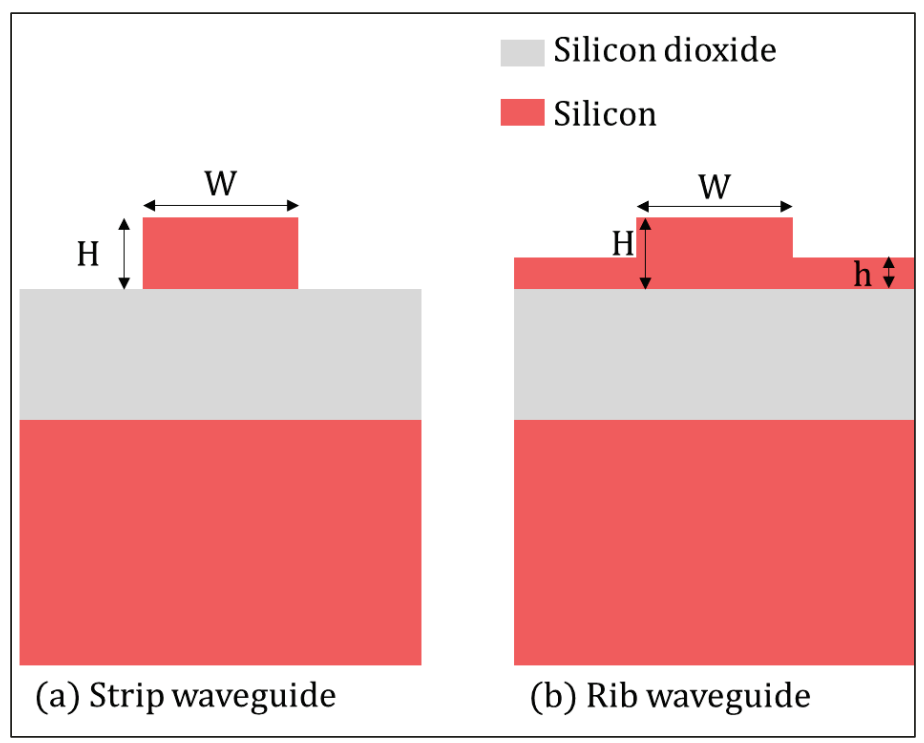

Figure 4.1 Schematic diagram depicting geometry of (a) Strip waveguide (b) Rib waveguide

In a rectangular waveguide there exist two families of modes, the $\mathrm{HE}$ mode and the EH modes, which are polarized in the TE or TM directions [21], respectively. The two modes are referred to as $\mathrm{E}^{\mathrm{x}}$ or $\mathrm{E}^{\mathrm{y}}$, based upon their maximum polarization direction. The modes are referred to as- $\mathrm{E}_{\mathrm{p}, \mathrm{q}}^{\mathrm{x}}$ or $\mathrm{E}_{\mathrm{p}, \mathrm{q}}^{\mathrm{y}}$, where $\mathrm{p}$ and $\mathrm{q}$ represent the number of field maxima in the $\mathrm{x}$ and $\mathrm{y}$ directions, respectively. These modes are also referred to as $\mathrm{HE}_{\mathrm{p}, \mathrm{q}}$ and $\mathrm{EH}_{\mathrm{p}, \mathrm{q}}$ modes. The fundamental modes are denoted as $\mathrm{E}_{1,1}^{\mathrm{x}}$ or $\mathrm{E}_{1,1}^{\mathrm{y}}$ [21]. Figure 4.2 depict the first two mode profiles of a strip waveguide. 


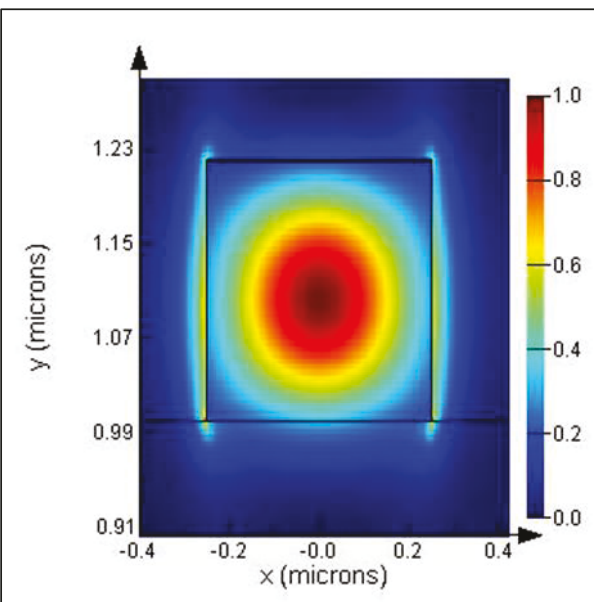

(a)

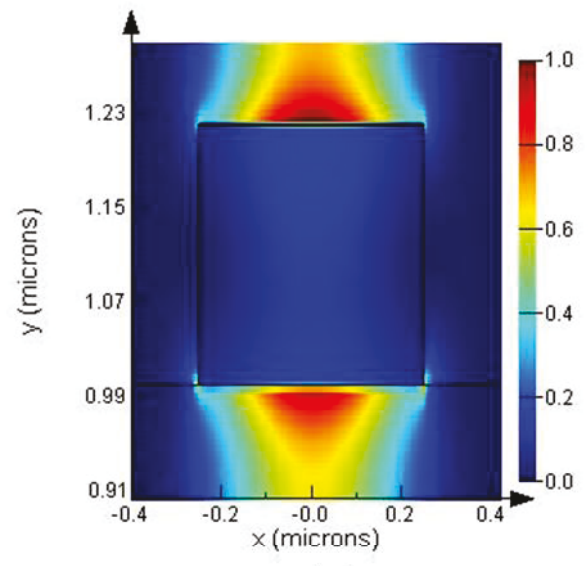

(c)

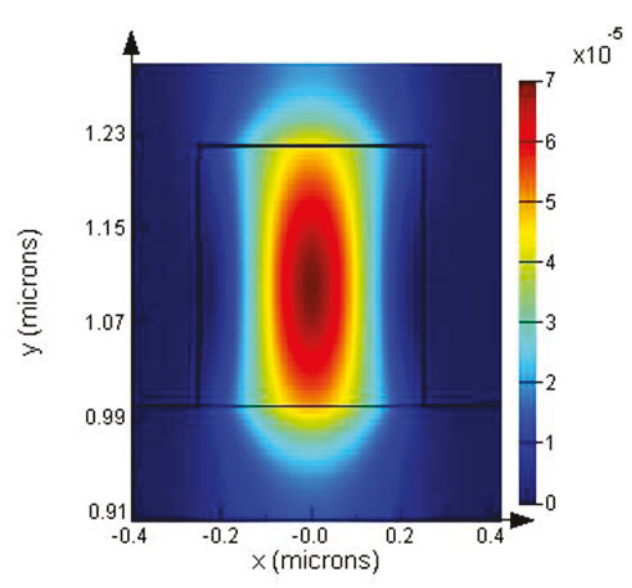

(b)

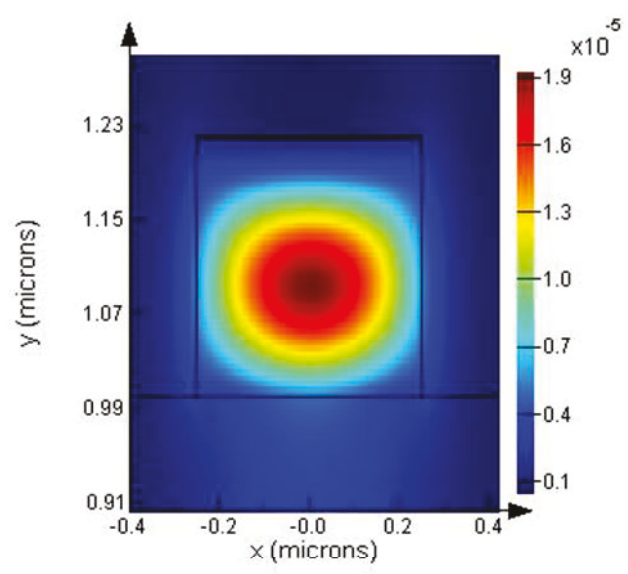

(d)

Figure 4.2. Mode profiles of the two guided mode in strip waveguide (a) Electric field intensity profile for Mode-1 (b) Magnetic field intensity profile for Mode-1 (a) Electric field intensity profile for Mode-2 (b) Magnetic field intensity of Mode-2 


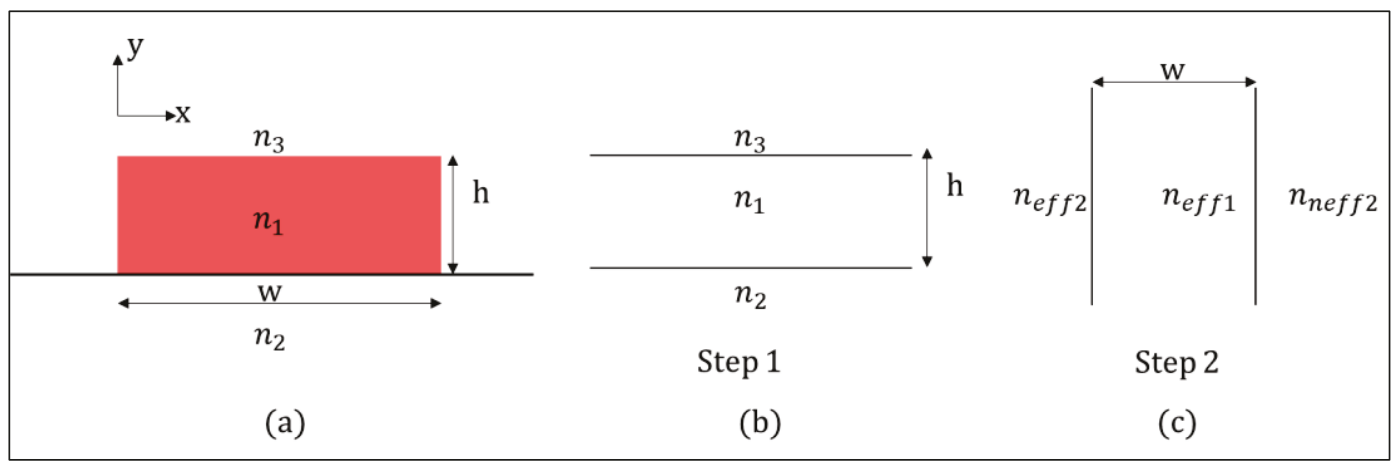

Figure 4.3. (a) General diagram of strip waveguide (b)- (c) decomposition into two imaginary planar waveguides (Ref.[21])

To solve for the effective index, we must know the refractive indices of the core $\left(n_{1}\right)$, cladding $\left(n_{2}, n_{3}\right)$ and the waveguide dimensions $(\mathrm{w}, \mathrm{h})$. We solve for the propagation constant of the guided mode by considering the two-dimensional waveguide as a combination of two planar waveguides- one horizontal and the other vertical (Figure 4.3). To find the effective index of the propagating mode in the strip waveguide, the planar waveguide eigenvalue equation is solved in the horizontal direction first and then in the vertical direction. The effective index of the core and cladding found in Step-1 (Figure 4.3 (b)) are assigned to the respective layers for the vertical structure in Step-2 (Figure 4.3 (c)). Before solving, one must know the polarization to solve for. For the TE polarized mode (with electric field polarized in $\mathrm{x}$ direction) we first use the TE eigenvalue equation [21]. We then use the TM eigenvalue equation to solve the vertical three-layer planar waveguide. We use the TM eigenvalue equation because the field is polarized in the TM direction with respect to the imaginary vertical waveguide. 
The horizontal planar waveguide (Figure 4.3 (b)) is solved for the propagation angle $\theta_{1}$ using the asymmetrical TE eigen-value equation (4.1) for $\mathrm{m}=0$ [21].

$$
\begin{gathered}
\mathrm{k}_{0} \mathrm{n}_{1} \mathrm{~h} \cos \theta_{1}-\mathrm{m} \pi=\tan ^{-1}\left[\frac{\sqrt{\sin ^{2} \theta_{1}-\left(\frac{\mathrm{n}_{2}}{\mathrm{n}_{1}}\right)^{2}}}{\cos \theta_{1}}\right]+ \\
\tan ^{-1}\left[\frac{\sqrt{\sin ^{2} \theta_{1}-\left(\frac{\mathrm{n}_{3}}{\mathrm{n}_{1}}\right)^{2}}}{\cos \theta_{1}}\right]
\end{gathered}
$$

The propagation constant $\beta$, is calculated from equation (4.2) by replacing the propagation angle, $\theta_{1}$, with the value found from equation (4.1)-

$$
\beta=\mathrm{n}_{1} \mathrm{k}_{0} \sin \theta_{1}
$$

And, the effective index of the core region is calculated as-

$$
\mathrm{n}_{\mathrm{eff} 1}=\frac{\beta}{\mathrm{k}_{0}}=\mathrm{n}_{1} \sin \theta_{1}
$$

For a rib waveguide, the effective index of the planar regions flanking the 'rib' are calculated by solving the asymmetrical TE eigenvalue equation for the smaller height. This results in the effective index region of the planar region $=\mathrm{n}_{\mathrm{eff} 2}$. Whereas, for a strip waveguide $\mathrm{n}_{\text {eff2 }}=n_{3}$. We proceed to solve the planar waveguide given in Figure 4.3 (c) with the effective indices calculated in the previous part, $\mathrm{n}_{\mathrm{eff} 1}$ and $\mathrm{n}_{\mathrm{eff} 2}$. The TM eigenvalue equation, equation (4.4) for the symmetrical waveguide is solved for $\theta_{\mathrm{wg}}$. This is because for either strip or rib waveguide, both the left and the right cladding index equals $\mathrm{n}_{\mathrm{eff} 2}$. 


$$
\mathrm{k}_{0} \mathrm{n}_{\mathrm{eff} 1} \mathrm{w} \cos \theta_{\mathrm{wg}}=2 \tan ^{-1}\left[\frac{\sqrt{\left(\frac{\mathrm{n}_{\text {eff1 }}}{\mathrm{n}_{\text {eff } 2}}\right)^{2} \sin ^{2} \theta_{\mathrm{wg}}-1}}{\left(\frac{\mathrm{n}_{\mathrm{eff2}}}{\left.\mathrm{n}_{\text {eff } 1}\right) \cos \theta_{\mathrm{wg}}}\right.}\right]
$$

From equation (4.4), the angle $\theta_{\mathrm{wg}}$, is obtained and the effective index of the waveguide is calculated as $\mathrm{n}_{\mathrm{wg}}=n_{\text {eff } 1} \cdot \theta_{\mathrm{wg}}$. While designing the grating couplers we must know the average effective average index of the grating structure. We attained the effective index for the unetched and the partially etched silicon using Lumerical ${ }^{\circledR}$ MODE Solutions.

\subsection{Effective Medium Theory}

Effective Medium Theory (EMT) was first given in 1956 by S. Rytov for his work on finely stratified medium [28] and it approximates the effective index of a medium with periodic sub-wavelength structures. EMT describes the interaction between an incident field and a subwavelength structure [31]. The effective medium properties are determined by approximating the electric field within a subwavelength

period, $\boldsymbol{\Lambda}$, as a constant and applying electromagnetic boundary conditions to the fields across the material interface [32]. Rytov's formulae are derived using a zeroorder approximation, i.e. assuming the ratio $\lambda / \Lambda \rightarrow \infty$ [17]. The effective refractive index for the equivalent homogenous medium is approximated for both the TE and TM polarizations are is discussed below- 


\section{A. Polarization parallel to the length of the alternating media:}

This is also known a Transverse Electric (TE) or p-polarization, where the electric field vector is perpendicular to the plane of incidence, as seen in Figure 4.4. For TE polarization, the electric field (E) is parallel to the periodic layers of the grating.

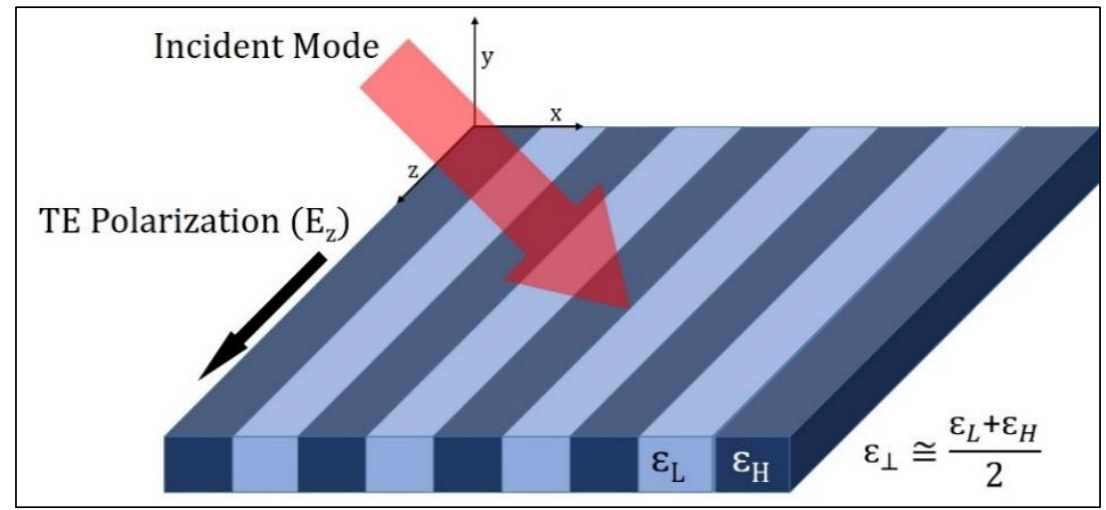

Figure 4.4. Effective Index for TE polarization (Ref. [34] )

Therefore, as per the boundary conditions, $\mathbf{E}$ must be continuous across the interfaces. Since, $\mathbf{E}$ is constant throughout the grating, $\Rightarrow \mathbf{E}_{\text {avg }}=\mathbf{E}_{0}$. The electric displacement vector $\mathbf{D}\left(\mathbf{C} / \mathbf{m}^{2}\right)$ includes the effect of electromagnetic waves on the matter [16]. D is calculated for the two periodic regions -

$$
\begin{gathered}
\mathrm{D}_{1}=\varepsilon_{1} \mathrm{E}_{0} \\
\mathrm{D}_{2}=\varepsilon_{2} \mathrm{E}_{0} \\
\mathrm{D}_{\text {avg }}=\mathrm{ff} \cdot \mathrm{D}_{1}+(1-\mathrm{ff}) \cdot \mathrm{D}_{2} \\
\mathrm{D}_{\text {avg }}=\mathrm{ff} \cdot \varepsilon_{1} \mathrm{E}_{0}+(1-\mathrm{ff}) \cdot \varepsilon_{2} \mathrm{E}_{0}
\end{gathered}
$$


The effective permittivity is defined as: -

$$
\begin{gathered}
\varepsilon_{\mathrm{TE}} \cong \frac{\mathrm{ff} \cdot \varepsilon_{1} \mathrm{E}_{0}+(1-\mathrm{ff}) \cdot \varepsilon_{2} \mathrm{E}_{0}}{\mathrm{E}_{0}} \\
\varepsilon_{\mathrm{TE}} \cong \mathrm{ff} \cdot \varepsilon_{1}+(1-\mathrm{ff}) \cdot \varepsilon_{2}
\end{gathered}
$$

Since,

$$
\mathrm{n}_{\mathrm{eff}}=\sqrt{\varepsilon_{\mathrm{eff}}}
$$

Therefore,

$$
\mathrm{n}_{\mathrm{TE}}^{2}=\mathrm{ff} \cdot \mathrm{n}_{1}^{2}+(1-\mathrm{ff}) \cdot \mathrm{n}_{2}^{2}
$$

This approximation if valid if the subwavelength period is less than $\frac{\lambda}{2 n}$, where $n$ is the refractive index of the medium and $\lambda / \mathrm{n}$ is the wavelength in the medium [32]. Since, $\mathrm{ff}=\frac{\mathrm{a}}{\Lambda}$ (where $\mathrm{a}=$ length of high index region, $\Lambda=$ grating period), the equation (4.12) is re-written as (4.13)-

$$
\mathrm{n}_{\mathrm{TE}}^{2}=\frac{\mathrm{a}}{\Lambda} \cdot \mathrm{n}_{1}^{2}+\left(1-\frac{\mathrm{a}}{\Lambda}\right) \cdot \mathrm{n}_{2}^{2}
$$


B. Polarization perpendicular to the length of the alternating media:

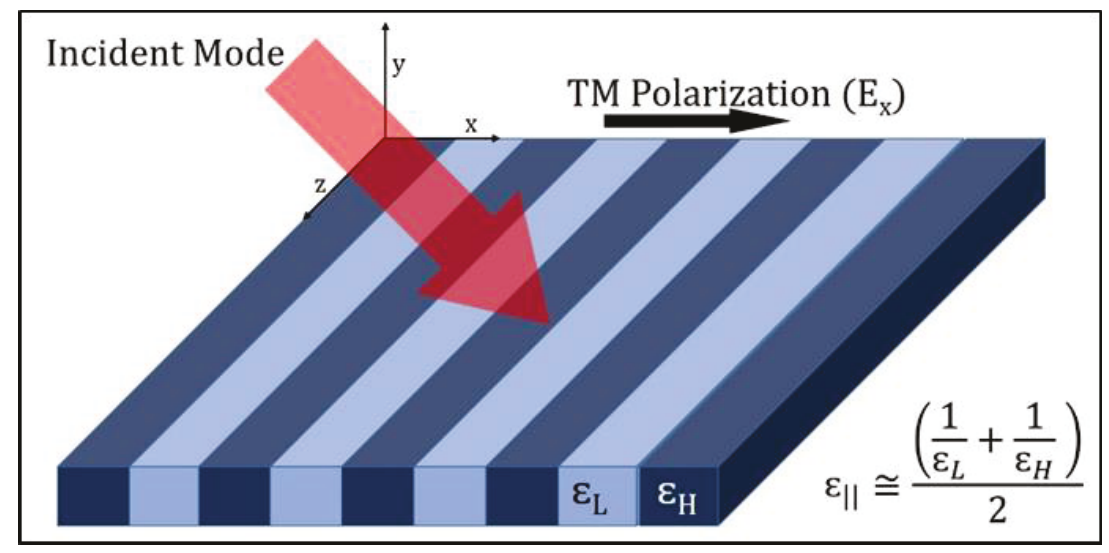

Figure 4.5. Effective Index for TM Polarization (Ref. [34])

In this polarization, the electric field vector is perpendicular to the grating. It is also referred to as the Transverse Magnetic (TM) or s-polarization, where the electric field vector is located in the plane of incidence. For TM polarization: $\mathbf{E}$ is perpendicular to the grating grooves. As per the boundary conditions $\mathbf{D}$ must be continuous across the interfaces. D has a nearly constant value throughout the grating, therefore, $\mathbf{D}_{\text {avg }}=\mathbf{D}_{0}$. Electric field in each region is given as: -

$$
\begin{gathered}
\mathrm{E}_{1}=\frac{\mathrm{D}_{0}}{\varepsilon_{1}} \\
\mathrm{E}_{2}=\frac{\mathrm{D}_{0}}{\varepsilon_{2}} \\
\mathrm{E}_{\mathrm{avg}}=\mathrm{ff} \cdot \mathrm{E}_{1}+(1-\mathrm{ff}) \cdot \mathrm{E}_{2} \\
\mathrm{E}_{\text {avg }}=\mathrm{ff} \cdot \frac{\mathrm{D}_{0}}{\varepsilon_{1}}+(1-\mathrm{ff}) \cdot \frac{\mathrm{D}_{0}}{\varepsilon_{2}}
\end{gathered}
$$




$$
\begin{gathered}
\frac{\mathrm{D}_{0}}{\varepsilon_{\mathrm{TM}}}=\mathrm{ff} \cdot \frac{\mathrm{D}_{0}}{\varepsilon_{1}}+(1-\mathrm{ff}) \cdot \frac{\mathrm{D}_{0}}{\varepsilon_{2}} \\
\frac{1}{\varepsilon_{\mathrm{TM}}}=\mathrm{ff} \cdot \frac{1}{\varepsilon_{1}}+(1-\mathrm{ff}) \cdot \frac{1}{\varepsilon_{2}}
\end{gathered}
$$

Since, $\varepsilon \propto \mathrm{n}^{2}$,

$$
\frac{1}{\mathrm{n}_{\mathrm{TM}}^{2}}=\mathrm{ff} \cdot \frac{1}{\mathrm{n}_{1}^{2}}+(1-\mathrm{ff}) \cdot \frac{1}{\mathrm{n}_{2}^{2}}
$$

For, $\mathrm{ff}=\frac{\mathrm{a}}{\Lambda}$, equation $(4.20)$ can be re-written as follows:-

$$
\frac{1}{\mathrm{n}_{\mathrm{TM}}^{2}}=\frac{\mathrm{a}}{\Lambda} \cdot \frac{1}{\mathrm{n}_{1}^{2}}+\left(1-\frac{\mathrm{a}}{\Lambda}\right) \cdot \frac{1}{\mathrm{n}_{2}^{2}}
$$

It is noted that for a given duty cycle $\mathrm{n}_{\mathrm{TE}}>\mathrm{n}_{\mathrm{TM}}$, implying that SWGs are intrinsically birefringent. For a low dielectric contrast system, the maximum difference in the effective indices of the two orthogonal polarizations is less than when compared to a high dielectric contrast system as seen in Figure 4.6. In Figure 4.6 (a), $\mathrm{n}_{1}=3.4777$ for silicon and $\mathrm{n}_{2}=1.45$ representing $\mathrm{SiO}_{2}$. In Figure $4.6(\mathrm{~b}), \mathrm{n}_{1}=$ 2.5 and $\mathrm{n}_{2}=1.45$. Since, $\mathrm{Si}-\mathrm{SiO}_{2} / \mathrm{Air}$ is a high dielectric contrast system, the difference in the effective indices of the TE and TM modes are high and need to be accounted for while designing a polarization independent/flexible grating coupler. 

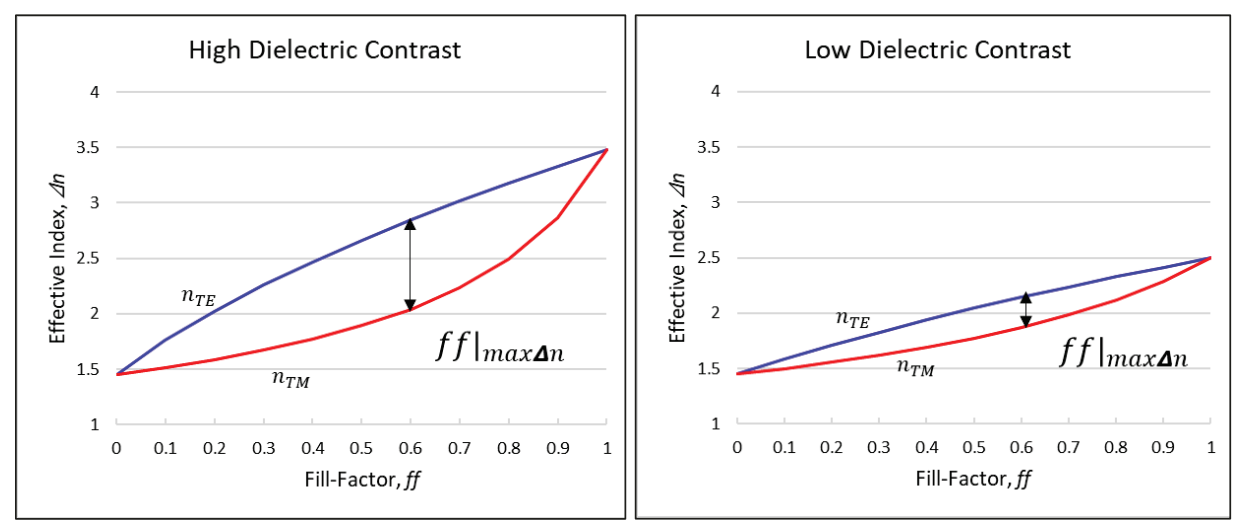

Figure 4.6. (a) Effective indices for TE and TM polarizations in a (a) high dielectric contrast medium (b) low dielectric contrast medium.

Though the EMT approximates the effective refractive index of the grating structure for the Bragg condition, the exact mode/ effective index of the modes propagating through the sub-wavelength structures are calculated using 3D FDTD Band Solver for the low and the high index regions assuming that the sub-wavelength structures constitute an infinite photonic crystal. To simulate this periodic structure the Bloch boundary condition are used. For simulation and optimizations of grating structure, the approximate refractive indices obtained using EMT are used to get the approximate grating parameters which are then further fine-tuned using simulations.

\subsection{Finite Difference Time Domain Method}

The Finite-Difference Time-Domain method (FDTD) is one of the numerical methods for solving electromagnetic problems. The technique was first proposed by K. Yee in 1966. The Maxwell equations which describe the fundamental laws of electromagnetism written as eq. (4.22) - (4.25). 


$$
\begin{gathered}
\nabla \times \boldsymbol{E}+\frac{\partial \boldsymbol{B}}{\partial t}=0 \\
\nabla \times \boldsymbol{H}-\frac{\partial \boldsymbol{D}}{\partial t}=\boldsymbol{J} \\
\nabla \cdot \boldsymbol{D}=\rho \\
\nabla \cdot \boldsymbol{B}=0
\end{gathered}
$$

Where, $\mathbf{E}$ and $\mathbf{H}$ are the electric and magnetic field vectors, respectively. $\boldsymbol{D}=\varepsilon \boldsymbol{E}$ is the electric displacement vector and $\boldsymbol{B}=\mu \boldsymbol{H}$ is the magnetic induction vector. The quantities $\boldsymbol{\rho}$ and $\mathbf{J}$ are the electric charge density and the electric current density, respectively.

The equations (4.22) - (4.25) can be re-written for $\boldsymbol{\rho}=0$ and $\mathbf{J}=0$, since our system is neutral in charge. This proposes the existence of the electromagnetic fields in the absence of electric charges.

$$
\begin{gathered}
\frac{\partial \mathbf{H}}{\partial \mathrm{t}}=-\frac{1}{\mu_{0}} \boldsymbol{\nabla} \times \mathbf{E} \\
\frac{\partial \mathbf{E}}{\partial \mathrm{t}}=\frac{1}{\varepsilon_{0}} \boldsymbol{\nabla} \times \mathbf{H}
\end{gathered}
$$

In a 1D-case, for a plane wave propagating in z-direction equation (4.26) and (4.27) can be re-written as-

$$
\begin{aligned}
& \frac{\partial \mathbf{E}_{\mathbf{x}}}{\partial \mathrm{t}}=-\frac{1}{\varepsilon_{0}} \frac{\partial \mathbf{H}_{\mathbf{y}}}{\partial \mathrm{t}} \\
& \frac{\partial \mathbf{H}_{\mathbf{y}}}{\partial \mathrm{t}}=-\frac{1}{\mu_{0}} \frac{\partial \mathbf{E}_{\mathbf{x}}}{\partial \mathrm{t}}
\end{aligned}
$$


From (4.28) and (4.29) we observe that the change in Electric field (with time) is dependent on the change in the Magnetic field in space. Yee's numerical method discretizes the Maxwell's equations in time and space. To approximate Maxwell's equation in space and time using Yee's algorithm, one first calculates all $\mathbf{H}$ field values, then all $\mathbf{E}$ field values. This method is widely used for solving Maxwell's equation in complex geometries. The accuracy and precision of the simulation results from FDTD simulations are highly dependent on the mesh size (Figure 4.7) .The Ecomponents are in the middle of the edges and the H-components are in the center of the face of a 3-D structure formed by a single mesh unit [33]. Hence, it is crucial to carefully select the mesh size. The space grid size must be such that over one mesh increment the electromagnetic field does not change significantly [33]. Smaller grid size mesh provides results with higher accuracy. For 2D-FDTD simulations, we used the mesh size of $\Delta x=5 \mathrm{~nm}, \Delta y=10 \mathrm{~nm}, \Delta z=40 \mathrm{~nm}$. The minimum feature size was limited to $25 \mathrm{~nm}$ along the $x$-direction, therefore a smaller mesh of $5 \mathrm{~nm}$ was selected. First, a smaller mesh size was chosen, but the selected mesh of $5 \mathrm{~nm}$ gave results accurate up to 2 decimal places and shorter simulation time, therefore $\Delta x=5 \mathrm{~nm}$, was selected. The silicon thickness size along $y$-direction was $220 \mathrm{~nm}$, therefore mesh size of $10 \mathrm{~nm}$, was sufficient. Along the $z$ - dimension of the grating was $12 \mu \mathrm{m}$ wide, therefore a larger mesh size of $40 \mathrm{~nm}$ was used.

For computational stability, it is necessary to satisfy a relation between the space increment and time increment $\Delta$ t. For constant $\epsilon$ (permittivity) and $\mu$ (permeability) computational stability requires that equation (4.30) is satisfied [33], where $\Delta \mathrm{x}, \Delta \mathrm{y}$ and $\Delta \mathrm{z}$ denote the grid size. 


$$
\sqrt{(\Delta \mathrm{x})^{2}+(\Delta \mathrm{y})^{2}+(\Delta \mathrm{z})^{2}}>\mathrm{c} \Delta \mathrm{t}=\sqrt{\frac{1}{\varepsilon \mu}} \Delta \mathrm{t}
$$

This requirement puts a restriction on $\Delta \mathrm{t}$ for the chosen mesh size$\Delta \mathrm{x}, \Delta \mathrm{y}$ and $\Delta \mathrm{z}$ [33]. Though, two-dimensional FDTD simulation requires less time, three-dimensional FDTD simulation may take hours to complete based upon the number of cores in the computer. For the simulation of devices for this thesis, we used Lumerical ${ }^{\circledR}$ FDTD Solutions. The Perfectly Matched Layer (PML) boundary conditions were used for all the axis for 2D FDTD simulations. The PML boundary has a finite thickness which helps in the absorption of light with low reflections through impedance matching to the simulation region and the materials. For 3D FDTD simulation we again chose the PML boundary condition except along the yaxis, which was the axis of symmetry for the grating coupler. For TE polarized light, the y-min boundary condition is set to Anti-Symmetric, since it helped to reduce the simulation time, whereas for TM, all the boundary conditions are PML.

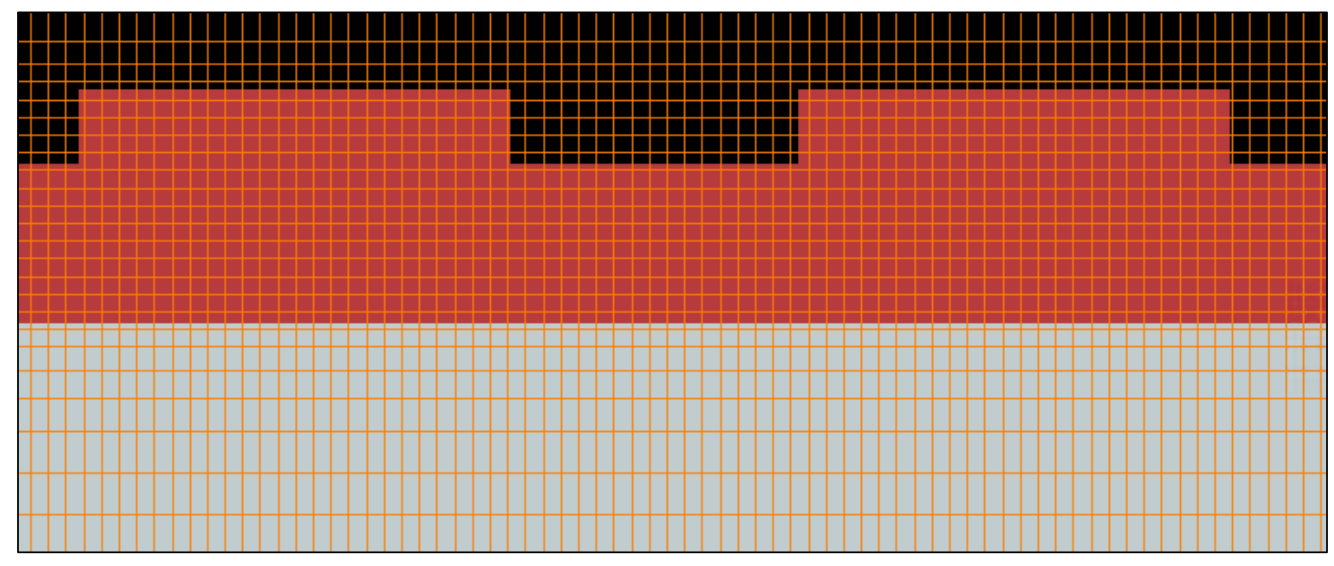

Figure 4.7. FDTD mesh for a grating coupler 


\section{Chapter 5}

\section{Polarization Flexible and Broadband}

\section{Grating Design}

This chapter outlines the details the process by which we designed the broadband and polarization flexible grating coupler which could efficiently couple both TE and TM polarized light into the silicon waveguide. The design objective was divided into two parts. First, to realise a polarization flexible design and second, to make the polarization flexible design broadband. We will first discuss the design of the polarization flexible grating coupler. This is followed by a discussion on the tunable grating parameters and their effect on the coupling efficiency, central wavelength and the coupling bandwidth. We then proceed to discuss the design methodology used to convert a shallow etched grating into a full-etch grating with broadband operation. The SWGC design is then modified for fabrication requirements and apodized to achieve a higher bandwidth and coupling efficiency. We finally conclude by discussing the results for the broadband and polarization insensitive SWGC. 


\subsection{Designing Uniform Sub-Wavelength Grating Coupler}

The simulation setup for the grating coupler consist of two FDTD ports, one of which are located at the silicon waveguide and the other at optical fiber. These ports act as a mode source, field monitor and mode expansion monitor. These ports were used to calculate the fundamental $\mathrm{TE}_{0}$ and $\mathrm{TM}_{0}$ mode of the silicon waveguide and the fiber. The ports along with the S-Parameter sweep were used to determine the S-parameters of the 2-port coupler. The modes in the fiber are calculated at a cross section normal to the fiber axis and then transmitted at an angle, $\theta$ to the surface normal of the grating coupler. Whereas for the planar waveguide, the modes are calculated for a silicon cross section of $220 \mathrm{~nm} \times 1 \mu \mathrm{m}$.

For the grating coupler in the out-couple mode, the far-field angle is found at the $1550 \mathrm{~nm}$ wavelength. The far-field is the angle at which most of the incident light is radiated/diffracted out from the grating structure. For an efficient grating coupler, there is only one dominant far-field angle for any given wavelength, i.e. only one diffraction order, while all others are suppressed by an order of a magnitude. For a grating coupler that depicts far-field with more than one diffraction/far-field angle result in a low or diffused directionality of the radiated power, thereby reducing the overall CE. The diffraction order can be calculated by substituting the variables in the Bragg condition (eq. 3.6) with the corresponding optimized grating parameters.

\section{Design of Polarization Independent Grating Coupler}

We begin with the design of polarization independent grating coupler. The polarization independent design is realized by partially etching silicon with $\mathrm{t}_{\mathrm{Si}}=$ 
220nm. For a polarization independent grating coupler, the Bragg condition (eq. (3.6)) must be satisfied for both TE and TM polarization of the guided mode. Adopting the methodology proposed by Tang and Dai we re-write the Bragg condition, eq. (3.6) for TE and TM with $\mathrm{m}=1$ and $\mathrm{m}=-1$ respectively, which yields equations (5.1) and (5.2). The grating pitch is calculated from equation (5.3), which is the result of the addition of equations (5.1) and (5.2)-

$$
\begin{array}{ll}
\text { TE: } & \Lambda\left(n_{\text {avg }}^{T E}{ }_{\text {eff }}^{T E}-n_{c} \times \sin \theta\right)=\lambda \\
T M: \quad & \Lambda\left(n_{\text {avg }}^{T M}+n_{c} \times \sin \theta\right)=\lambda \\
& n_{\text {avg }_{\text {eff }}^{T E}}^{T E}+n_{\text {avg }}^{T M f}=\frac{2 \lambda}{\Lambda}
\end{array}
$$

where, $\mathrm{n}_{\text {avg }}$ ff for TE or TM are given by-

$$
n_{\operatorname{avg}_{e f f}}=f f \cdot n_{e f f H}+(1-f f) \cdot n_{e f f L}
$$

For an etch depth, ed $=70 \mathrm{~nm}$ and fill-factor, $\mathrm{ff}=0.5$, the effective index of the unetched and partially etched Silicon at $1550 \mathrm{~nm}$ were found using Lumerical ${ }^{\circledR}$ Mode Solutions and are listed in Table 5-1,.

Table 5-1. Effective indices for the TE and TM polarization at $1550 \mathrm{~nm}$

\begin{tabular}{|c|c|c|c|}
\hline Polarization & $\begin{array}{c}\mathbf{n}_{\text {eff } 1} \text { for } \mathbf{t}_{\mathbf{S i}}= \\
\mathbf{2 2 0 n m}\end{array}$ & $\begin{array}{c}\mathbf{n}_{\text {eff }} \text { for } \mathbf{t}_{\mathbf{S i}}= \\
\mathbf{1 5 0 n m}\end{array}$ & $\mathbf{n}_{\text {avg }_{\text {eff }}}$ \\
\hline TE & 2.845578 & 2.54109 & 2.693334 \\
\hline TM & 2.064343 & 1.620891 & 1.842617 \\
\hline
\end{tabular}




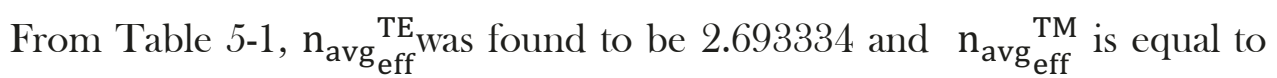
1.842617 for $\mathrm{n}_{\mathrm{c}}=1.45$. Equation (5.3) was solved for $\lambda=1550 \mathrm{~nm}$ and the approximate value of the grating period $(\Lambda)$ was calculated to be $683.42 \mathrm{~nm}$.

For the given grating period, $\Lambda=683.42 \mathrm{~nm}$, the angle of incidence, $\theta$ was calculated using equation (5.1) to be $17.056^{\circ}$. The grating coupler resulted behaved as a polarization splitter, where the TE and TM polarized modes were coupled towards opposite directions. The schematic diagram for the partially etched polarization independent and polarization splitting grating coupler is illustrated in Figure 5.1. The actual simulation structure is illustrated in Figure 5.2. Please refer to Appendix A for the Lumerical ${ }^{\circledR}$ script files to generate the grating structure for the given parameters. To further optimize the device performance, we run parametric sweeps for the tunable grating parameters.

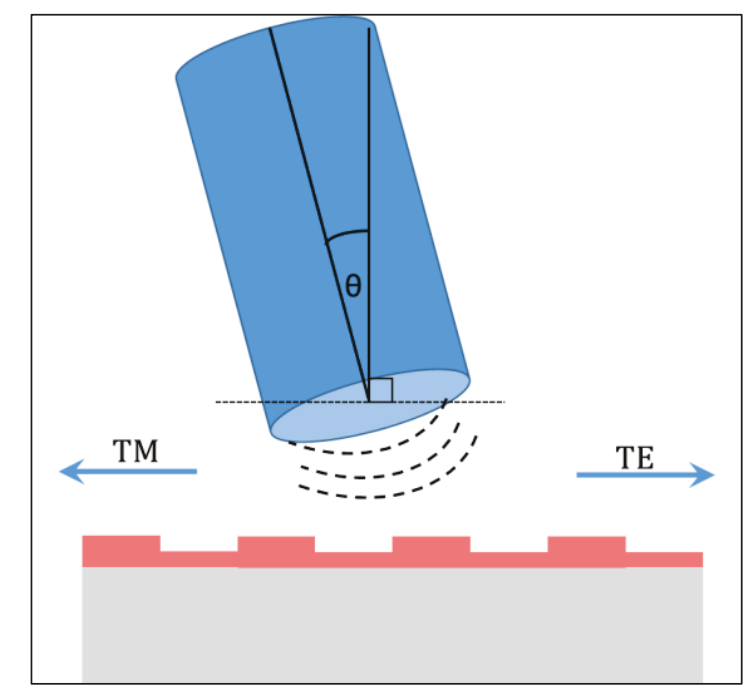

Figure 5.1. Schematic diagram for the polarization independent grating coupler design 


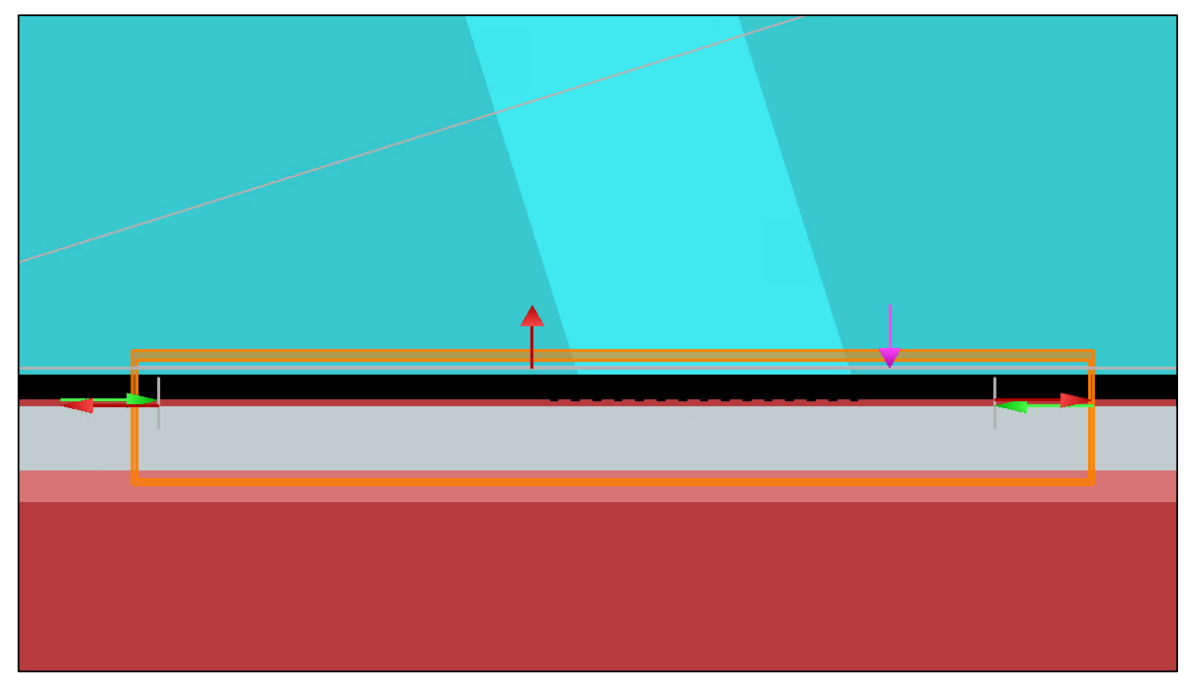

Figure 5.2. Schematic for the 2D simulation of the grating coupler

\subsubsection{Parametric Sweeps for Polarization Independent Design}

The initial grating coupler design theoretically designed was simulated using 2D-FDTD simulation to obtain the figure of merit (FOM). The parameters of this initial grating coupler are listed in Table 5-2. By simulating the device as an outcoupler, the far-field angle of incidence was found to be $17.4^{\circ}$, which is close to the calculated value of $17.056^{\circ}$.

Table 5-2. Initial grating coupler parameters

\begin{tabular}{|c|c|c|}
\hline$\Lambda(\mathrm{nm})$ & ff & ed (nm) \\
\hline 683.42 & 0.5 & 70 \\
\hline
\end{tabular}

The transmission spectrum of the initial grating coupler is illustrated in Figure 5.3. Though both the TE and TM polarized light were successfully coupled in, but the maximum transmission of $\mathrm{TE}$ is greater than $\mathrm{TM}$ by a factor of 1.275 times. 
Furthermore, the central wavelength for TM is offset by almost $25 \mathrm{~nm}$ from the idealdesired value of $1.55 \mu \mathrm{m}$. For the initial grating coupler, the coupling efficiencies for TE and TM polarization are $-2.93 \mathrm{~dB}$ and $-4.69 \mathrm{~dB}$, respectively at $1.55 \mu \mathrm{m}$, resulting in polarization dependent loss (PDL) of $-1.75 \mathrm{~dB}$ at $1.55 \mu \mathrm{m}$.

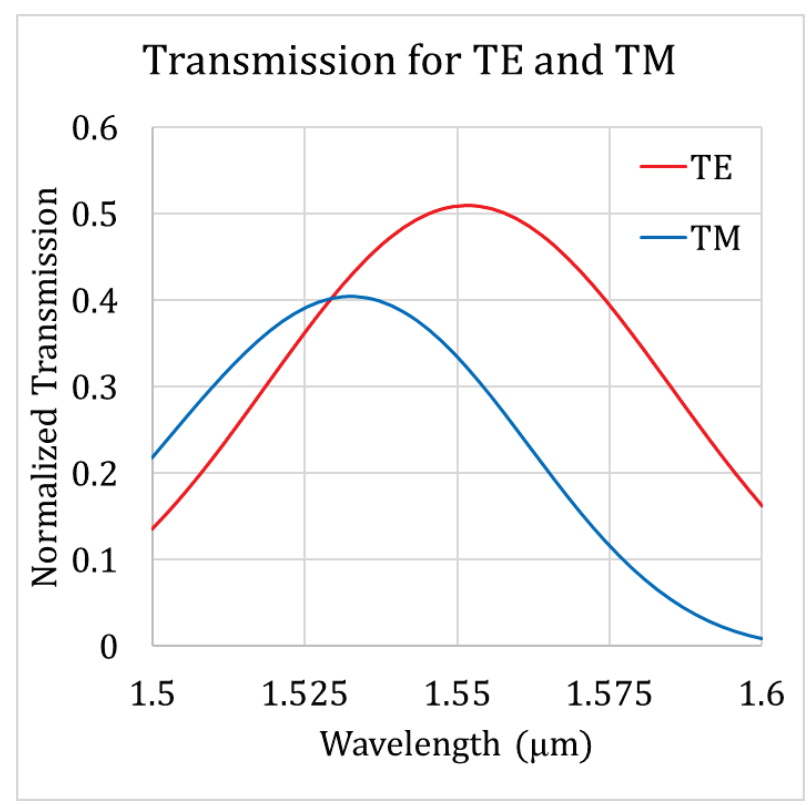

Figure 5.3. Transmission spectrum for the initial grating design

For a truly, polarization independent design, the PDL $\rightarrow 0$ at a given wavelength.

To achieve near zero polarization dependent losses, we optimize the grating parameters -etch depth, grating period and fill-factor. In the following sections, we discuss how each grating parameter alters the central wavelength and maximum transmission of the grating coupler. 


\section{A. Fill-Factor/ Duty Cycle}

From equation (5.4) it is known that the average effective index, $\mathrm{n}_{\mathrm{avg}} \mathrm{eff}_{\mathrm{f}}$ of the grating structure is directly proportional to the duty cycle. An increase in the fillfactor(ff) would result in an increase in $\mathrm{n}_{\mathrm{avg}} \mathrm{eff}$. The change in the effective index of the grating, also shifts the central wavelength as per the Bragg condition, equation (3.6). The transmission spectrum for TE and TM polarized light with varying fillfactors are plotted in Figure 5.4 and Figure 5.5, respectively.

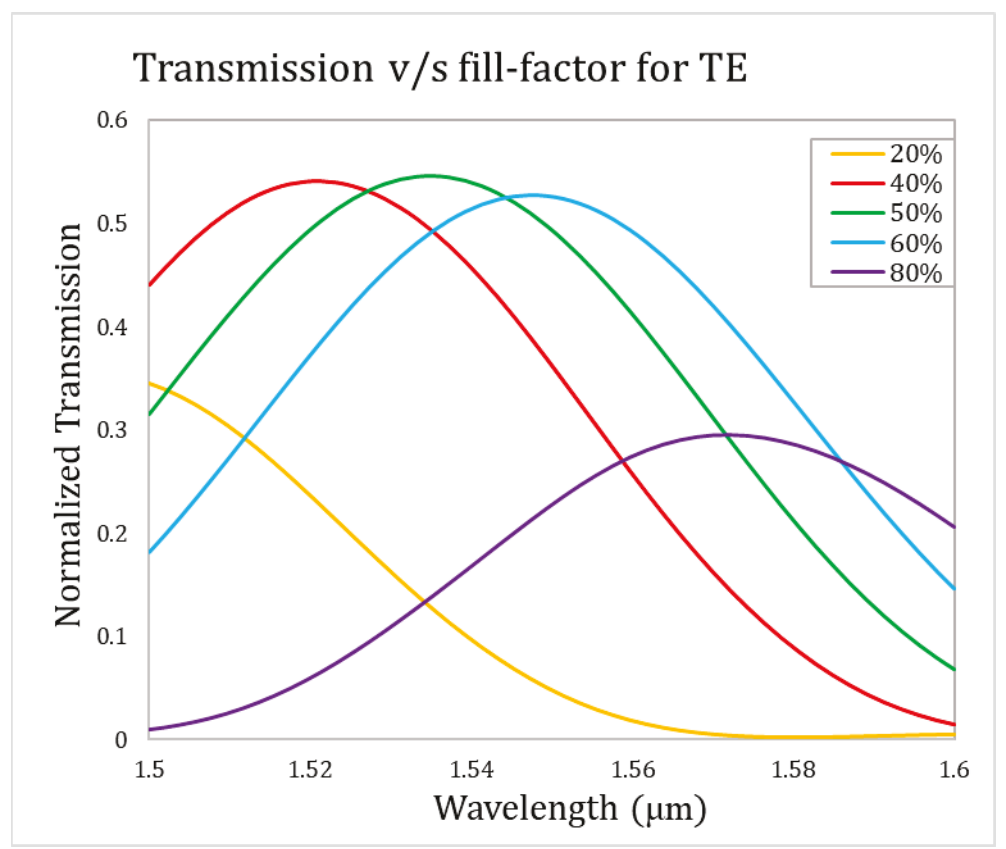

Figure 5.4. Variation of Transmission with fill-factor of grating for TE polarized light 


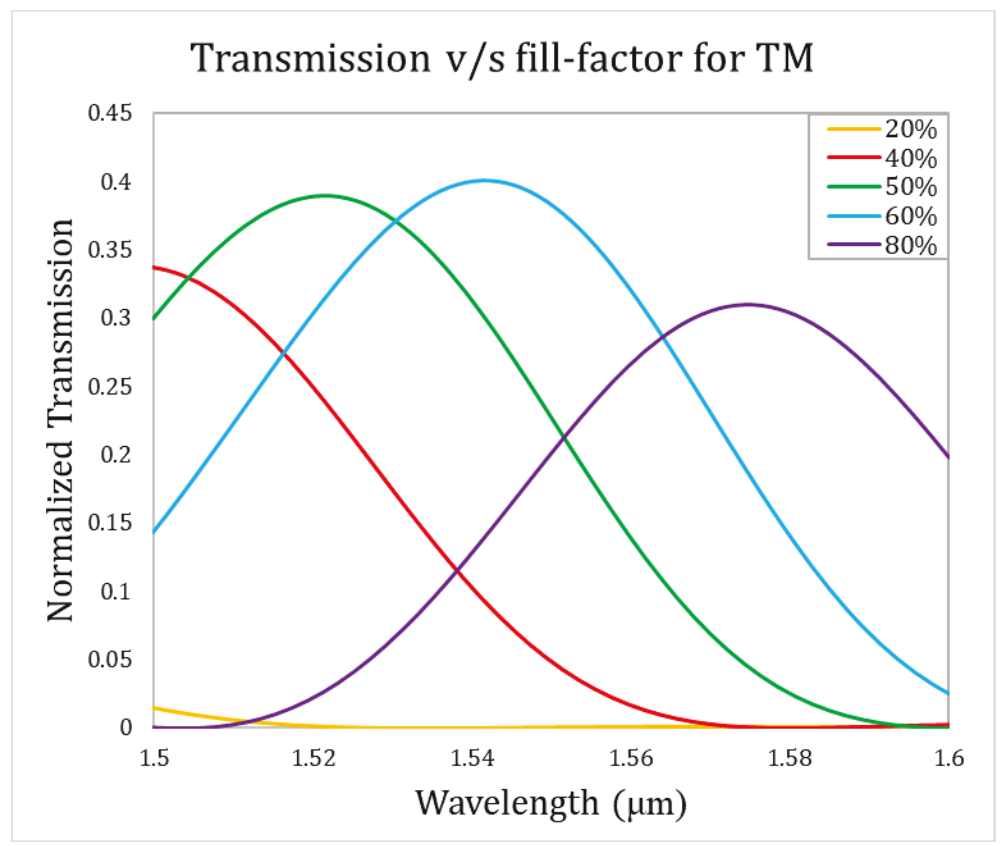

Figure 5.5. Variation of Transmission with fill-factor of grating for TM polarized light
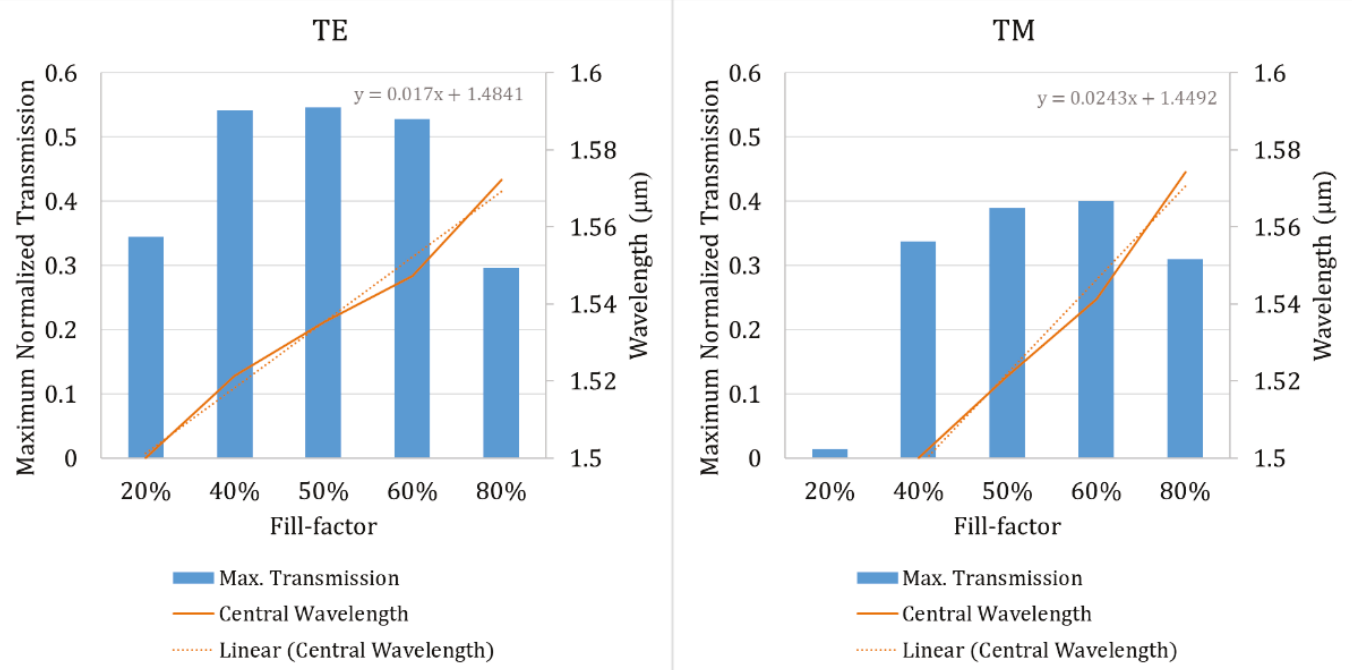

Figure 5.6. Variation of maximum transmission and wavelength with fillfactor 
It was observed that an increase in the fill-factor modifies the central wavelength and the maximum transmission for both polarizations. Figure 5.6 details the variation of the maximum transmission and central wavelength for each value of fill-factor. It is evident that for each fill-factor, the maximum transmission for TE is always greater than for TM. For TE polarized light, the maximum transmission is observed at $\mathrm{ff}=50 \%$ and $\mathrm{ff}=60 \%$ for $\mathrm{TM}$. Deviation from these fill-factor values result in a decrease in the maximum transmission. Furthermore, increase in the fill-factor also shifts the central wavelength towards higher values. A steeper slope of the trend-line for TM implies that the change in central wavelength is more rapid for TM as compared to TE for the same change in fill-factor $(\Delta f f)$. A $10 \%$ increase in the fill-factor shifts the coupling wavelength by $17 \mathrm{~nm}$ and $24.3 \mathrm{~nm}$ for TE and TM, respectively.

\section{B. Grating Period}

The grating period $(\Lambda)$ alters the wavelength at which the Bragg condition( eq. (3.6) is satisfied and successful coupling takes place. As per equation (5.1) and (5.2) the central coupling wavelength $(\lambda)$ is directly proportional to the grating period. A parametric sweep on the grating period for both TE and TM polarization confirms this. Figure 5.7 and Figure 5.8 represent the variation of the transmission spectrum for an input grating coupler for TE and TM polarized light. For constant angle of incidence (17.4), fill-factor (0.5) and etch-depth $(70 \mathrm{~nm})$, it is observed that that the maximum transmission decreases with the increase in the grating period. As 
expected, the central coupling wavelength also experiences a shift towards higher values.

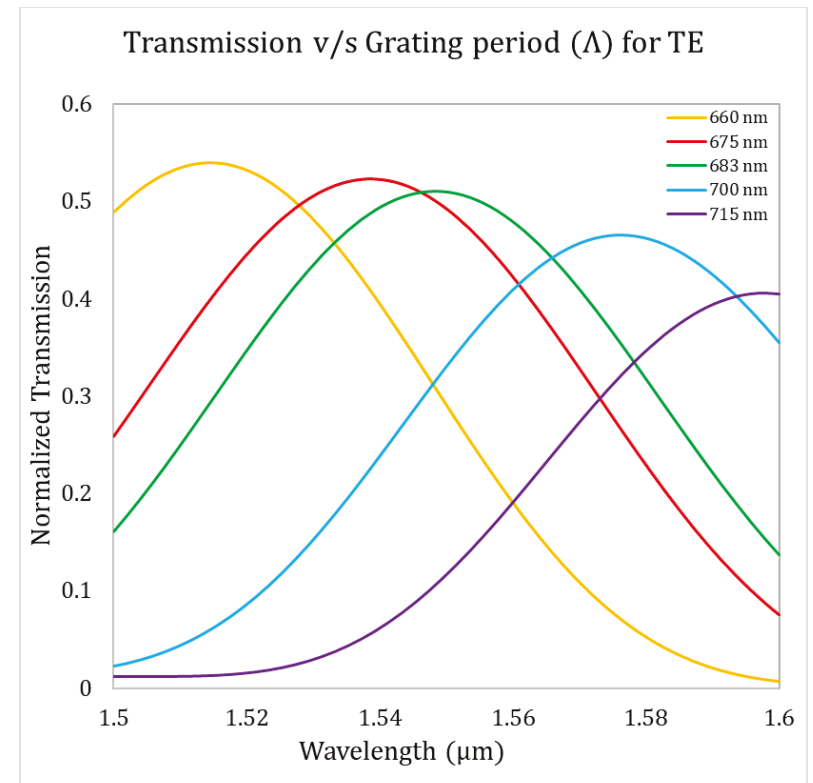

Figure 5.7. Variation of Transmission with Grating period for TE polarized light

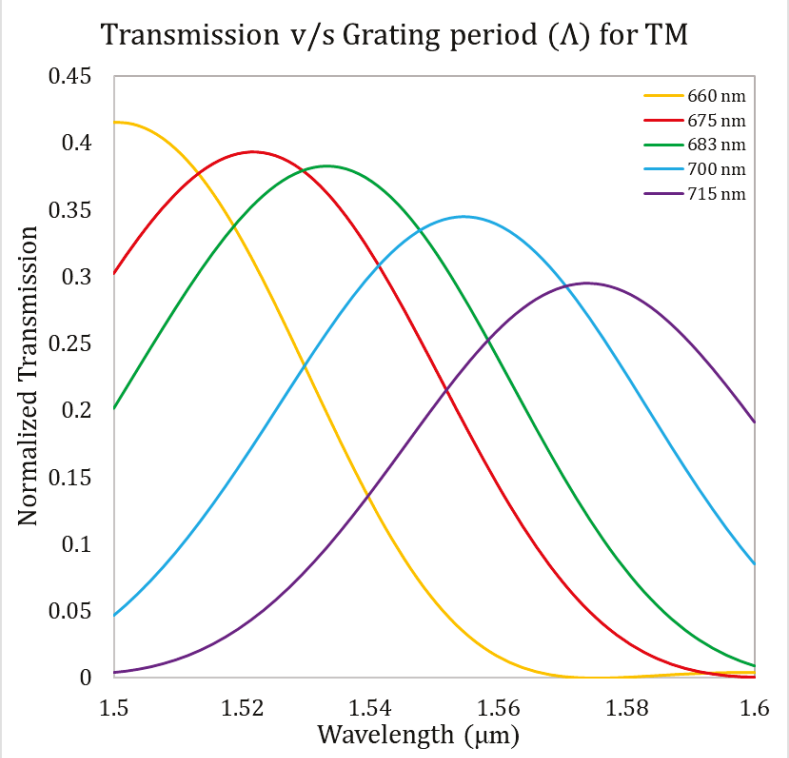

Figure 5.8. Variation of Transmission with Grating period for TM polarized light 

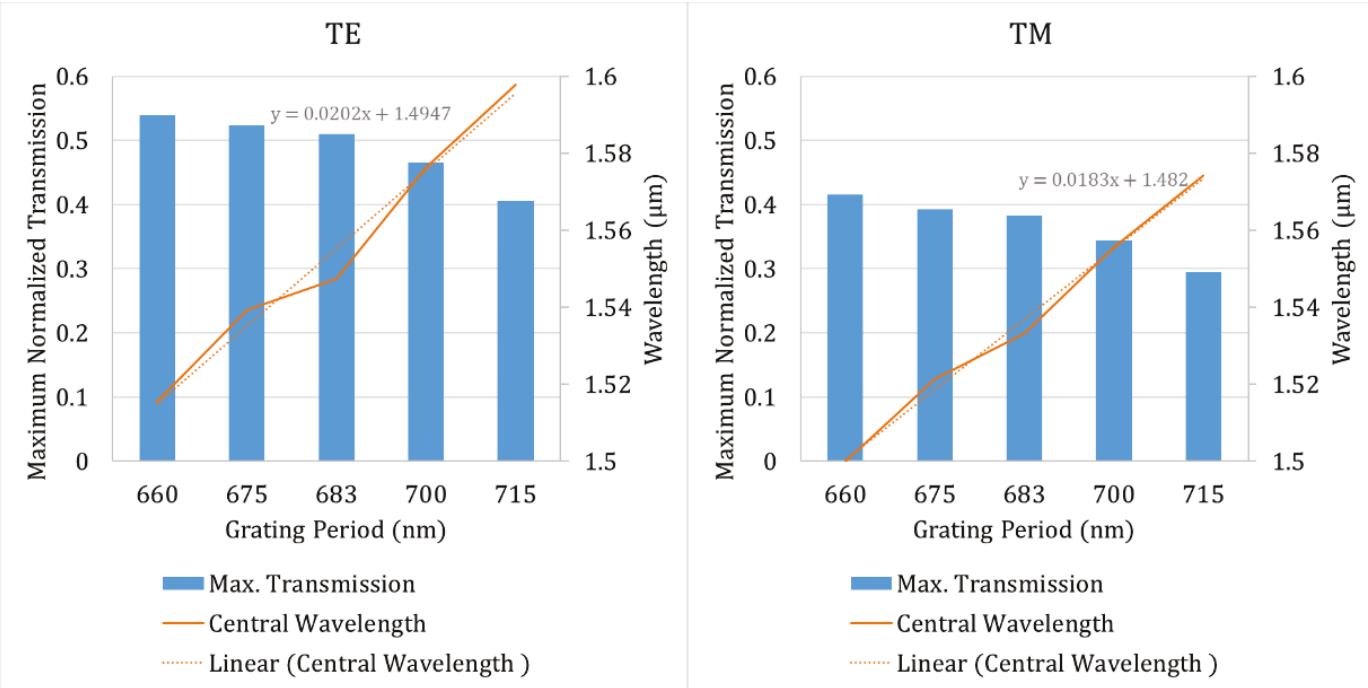

Figure 5.9. Variation of maximum transmission and wavelength with grating period

Figure 5.9, describes the variation in the maximum transmission and the central wavelength with the grating period. The maximum transmission reduces for both TE and TM with increase in the grating period, when all other grating parameters are kept constant. At the same time, the central wavelength shifts towards higher values. This shift is gradual for TM as compared to TE as seen from the slope of the trendline.

\section{Etch depth}

A parametric sweep on the etch depth was performed for the initial grating design. For both TE polarized light, the maximum in-coupling efficiency was observed for etch depth, ed $=70 \mathrm{~nm}$ as seen in Figure 5.10, whereas for TM ed = $70 \mathrm{~nm}$ and $90 \mathrm{~nm}$ result in near same maximum transmission (Figure 5.11). But, for the $90 \mathrm{~nm}$ etch, the central wavelength has a larger offset from the desired 
$1.55 \mu \mathrm{m}$ as compared to the $70 \mathrm{~nm}$ etch for TM. For the TE polarized light if the etch depth is deviated from the $70 \mathrm{~nm}$ value, the deviation in the maximum attainable transmission is greater as compared to TM. The etch depth has a stronger effect on TE polarized light as compared to TM polarized light. Etch depth has a reverse effect on the coupling wavelength as compared to the previously discussed grating parameters. As the etch depth is increased, the central coupling wavelength decreases, which is confirmed from the negative slope of the trend-line in Figure 5.12.

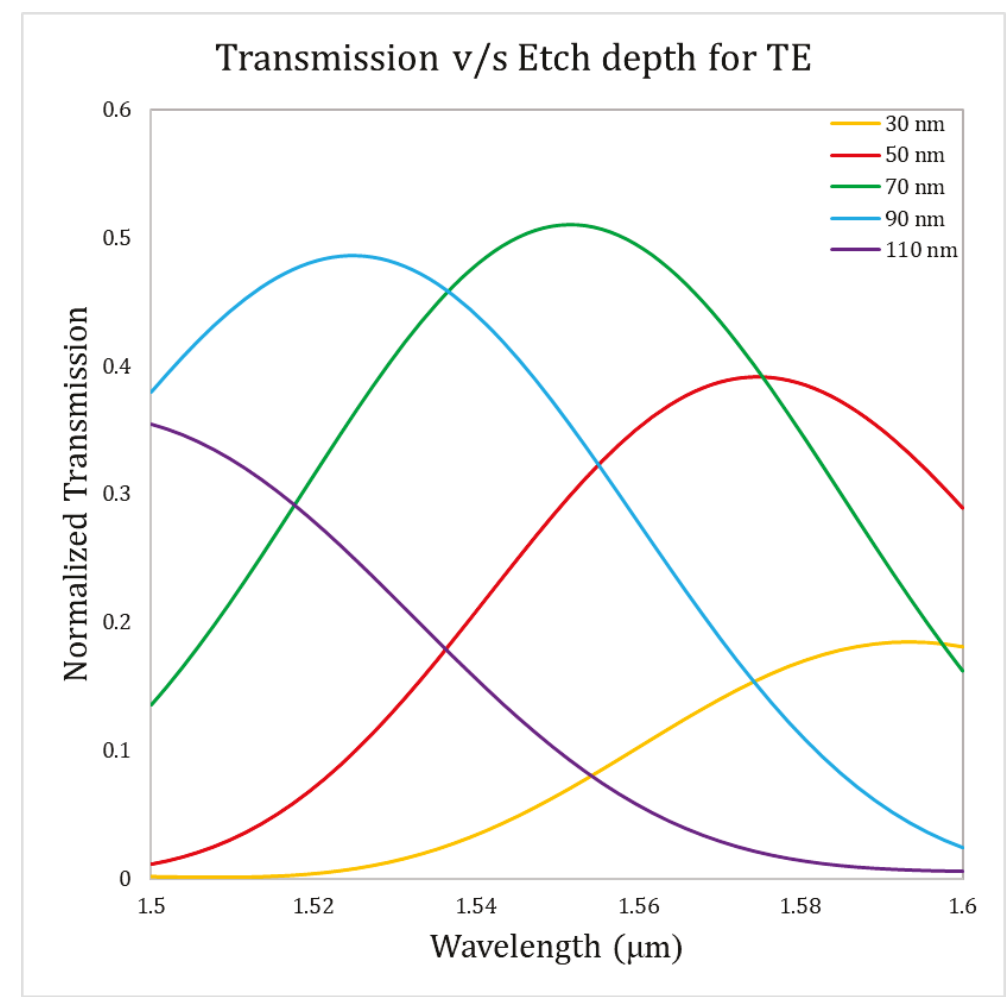

Figure 5.10. Variation of Transmission with etch depth for TE polarized light 


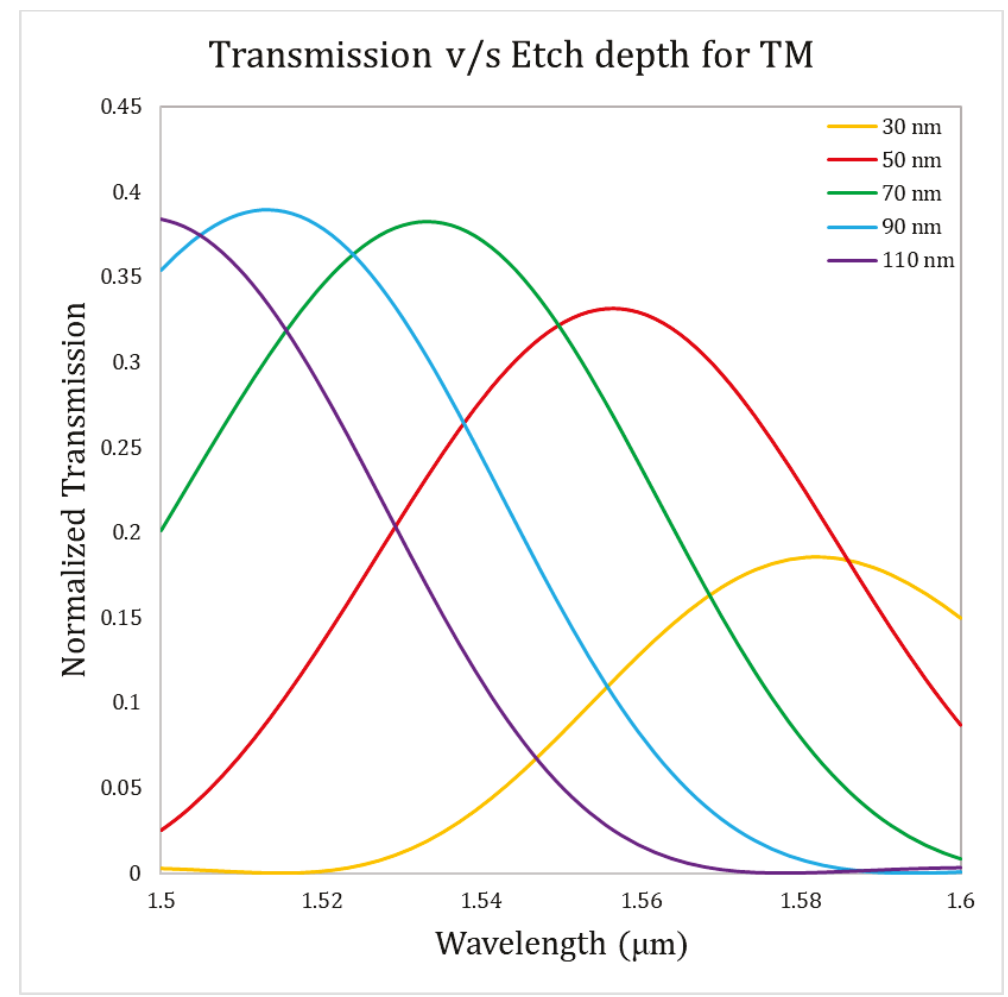

Figure 5.11. Variation of Transmission with etch depth for TM polarized light

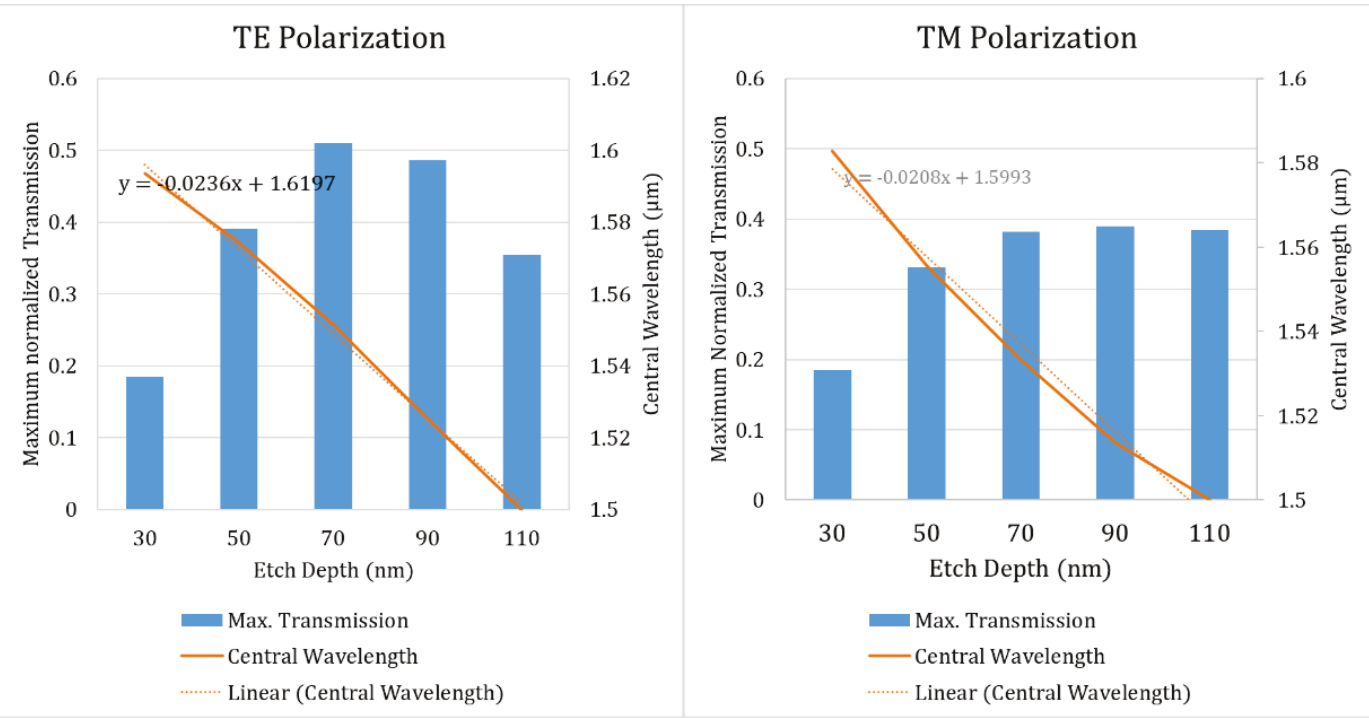

Figure 5.12. Variation in the maximum transmission and coupling wavelength with etch depth 
All the grating parameters discussed above act as tuning parameters for the grating performance. Based upon the effect of each grating parameter upon the maximum coupling efficiency, the bandwidth and the central wavelength, the design was optimized to achieve minimum PDL. The optimized design parameters are discussed in the following section.

\subsubsection{Simulation Results for Polarization Independent Design}

The design parameters for the optimized polarization independent grating coupler for partially etched silicon is given in Table 5-3 and the simulation results of the are depicted in Table 5-4. The PDL at $1.55 \mu \mathrm{m}$ are reduced to $0.87 \mathrm{~dB}$ at $1.55 \mu \mathrm{m}$, with maximum coupling efficiencies of $-2.95 \mathrm{~dB}$ and $-3.82 \mathrm{~dB}$ for TE and TM, respectively. A 1-dB coupling bandwidth of $45 \mathrm{~nm}$ and $37.5 \mathrm{~nm}$ were obtained for TE and TM, respectively. The 3-dB bandwidth of TM was attained to be $64.5 \mathrm{~nm}$, while for TE polarization, it was even higher at $79.3 \mathrm{~nm}$.

Table 5-3. Optimized design parameters for partially etched polarization independent and splitter design

\begin{tabular}{|c|c|c|c|c|}
\hline$\Lambda(\mathbf{n m})$ & $\mathbf{f f}_{1}$ & ed $(\mathbf{n m})$ & Angle of Incidence $(\boldsymbol{\theta})$ & Grating Length $(\boldsymbol{\mu m})$ \\
\hline 675 & 0.6 & 70 & $\mathrm{TE}=17.4^{\circ} \mid \mathrm{TM}=-17.4^{\circ}$ & 9.38 \\
\hline
\end{tabular}


Table 5-4. Simulation Results

\begin{tabular}{|c|c|c|c|c|}
\hline \multirow[t]{2}{*}{ Polarization } & \multicolumn{2}{|c|}{ Coupling Efficiency (dB) } & \multicolumn{2}{|c|}{ Bandwidth (nm) } \\
\hline & Maximum & @ 1550nm & $1-\mathrm{dB}$ & $3-\mathrm{dB}$ \\
\hline $\mathrm{TE}$ & -2.94dB@1547.37nm & -2.95 & 45 & 79.3 \\
\hline TM & -3.62 dB@1541.28 nm & -3.82 & 37.65 & 64.5 \\
\hline
\end{tabular}

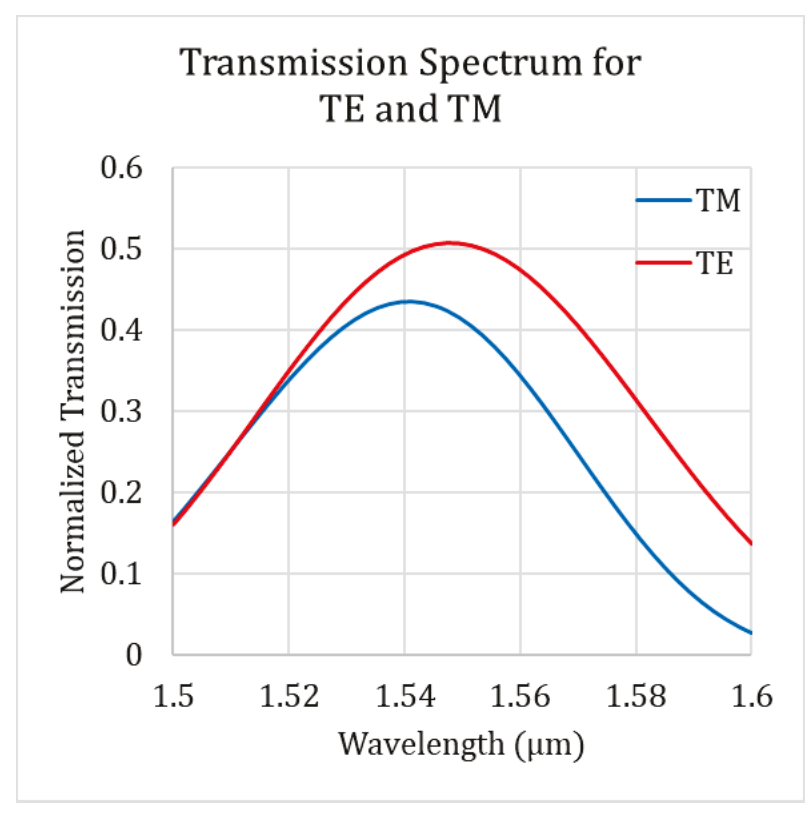

Figure 5.13. Transmission spectrum for TE and TM of the optimized polarization independent design 

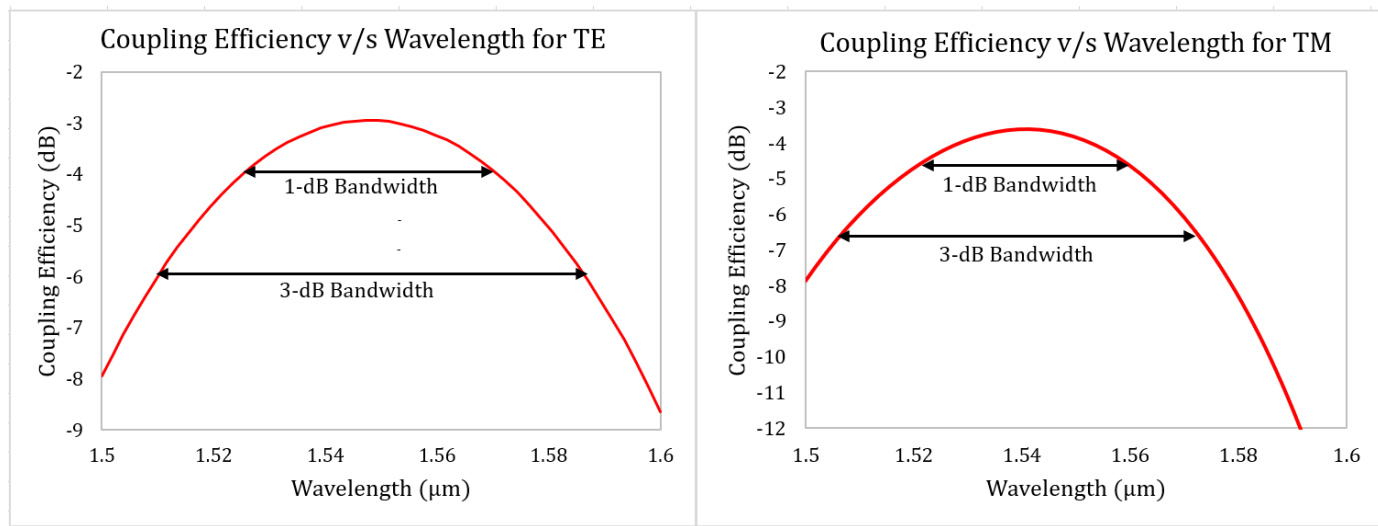

Figure 5.14. Coupling efficiency and bandwidths for TE and TM

Figure 5.13 depicts the transmission spectrum for both TE and TM polarization. Whereas, Figure 5.14 shows the coupling-efficiency and the bandwidth for the TE and TM polarization. It is evident from the graphs that the coupling bandwidth for TE is more than for TM.

\subsubsection{S-Parameter for Polarization Independent Design}

The S-parameter completely describes the system response to an input. We can predict the reflections and transmission for each port in an n-port device. For an $n$-port device, $S$-matrix is a $n \times n$ matrix where each element, such that $\mathrm{i} \neq \mathrm{j}, \mathrm{S}_{\mathrm{ij}}$ gives the transmission coefficients for power incident at port $\mathrm{i}$, and output received at port j. The diagonal elements of a $\mathrm{S}$-matrix, $\mathrm{S}_{\mathrm{ii}}$, gives the relation between the incident power and the back- reflection at the $\mathrm{i}^{\text {th }}$ port. By Principle Of Reciprocity, the transmission spectrum of the grating coupler while working as a input coupler or an output coupler must be identical, thus $S_{12}=S_{21}$. For the same coupler, the back- 
reflections, $S_{11}$ and $S_{22}$ must be as low as possible $(<-20 \mathrm{~dB}$ for practical purposes). As discussed in Section 3.1.4 - Back Reflections

are highly undesired and lead to Fabry-Perot oscillations in the device and must be suppressed to as low as possible.

Figure 5.15 and Figure 5.16 give the S-parameter for the TE and TM polarization respectively. It is evident from the two figures that $S_{12} \approx S_{21}$, hence implying the system is reciprocal. Furthermore since, $S_{11}=S_{22} \rightarrow 0$, the simulated backreflections in the system is suppressed to well below $-29 d B$ for TE and $-19.5 d B$ for TM, hence making this design ideal for application as a polarization independent and splitter coupler in any Photonic Integrated Circuit.

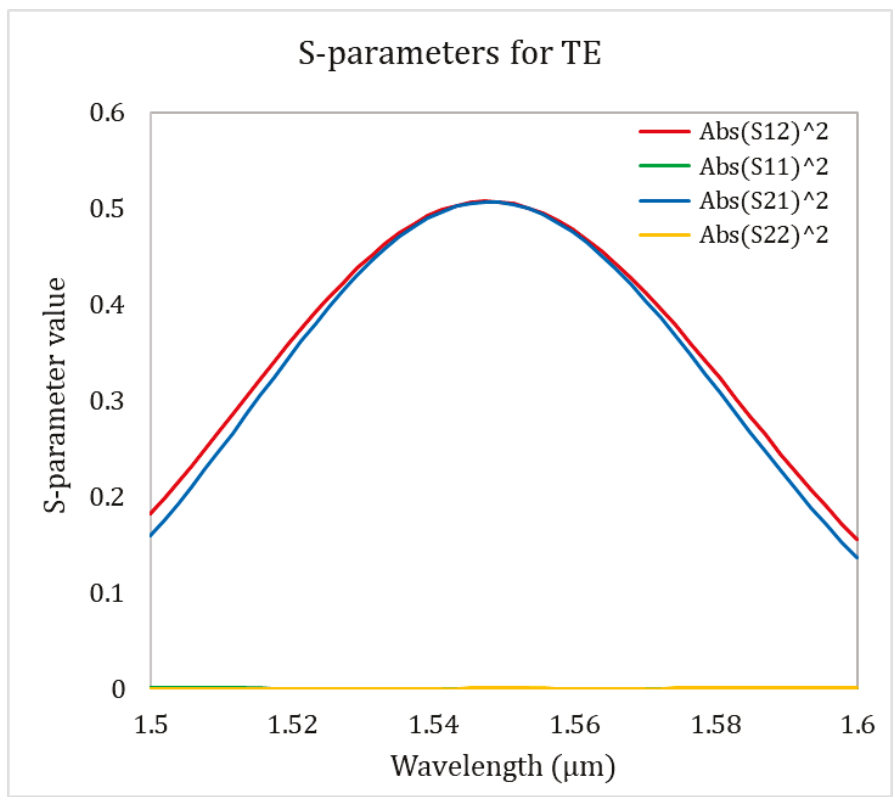

Figure 5.15. S-parameter for the TE polarization 


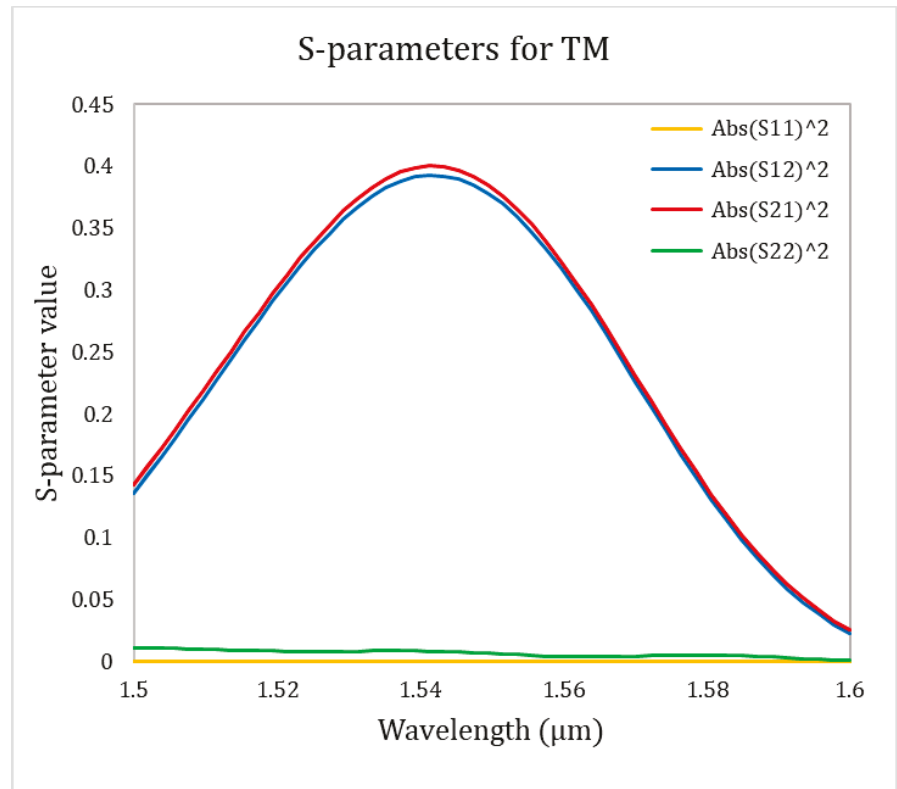

Figure 5.16. S-parameter for the TM polarization

We have devised a polarization independent design based on a $220 \mathrm{~nm}$ SOI wafer with a simulated maximum coupling efficiency of $-2.9 \mathrm{~dB}$ and $-3.8 \mathrm{~dB}$ for $\mathrm{TE}$ and TM, respectively. The 1-dB coupling bandwidths of the device are $45 \mathrm{~nm}$ and $37.65 \mathrm{~nm}$ for TE and TM, respectively. The 3-dB bandwidths reach up to $79.3 \mathrm{~dB}$ for TE and $64.5 \mathrm{~nm}$ for TM, which is the typical values for a grating coupler. In next section, we try to increase the 3-dB bandwidths for both TE and TM up to $100 \mathrm{~nm}$ and make the grating coupler broadband in operation.

\section{Design of Polarization Flexible and Broadband Grating Coupler}

In this section, we modify the polarization independent design to achieve a higher coupling bandwidth. The next step towards obtaining a larger bandwidth is to engineer the etch depth and the grating period. This was accomplished by first 
equating the refractive index of the shallow-etched grating to that of a full-etched grating for the $\mathrm{TM}_{0}$ polarization obtaining the approximate values of the fill-factors $\mathrm{n}_{\text {н }}$ and $\mathrm{n}_{\mathrm{L}}$. The conversion of the partially etched grating was only done for $\mathrm{TM}_{0}$, since it was the second highest mode in the silicon waveguide and the presence of $\mathrm{TM}_{0}$ indicated the fundamental guiding mode $\mathrm{TE}_{0}$ was also supported. Converting the partially-etched gratings to a full-etched SWG was followed by an increase in the grating period to further reduce the grating index. We know already know that $\mathrm{n}_{\mathrm{avg}}^{\mathrm{TM}}=1.842617$. Re-writing the Rytov's Formula for TM polarization from equation (4.21) to find the fill-factors of the high and the low index regions $\left(\mathrm{ff}_{\mathrm{H}}\right.$ and $\mathrm{ff}_{\mathrm{L}}$ ). The fill-factors in turn determine the effective index of the high and the low index regions. The completely etched regions have $\mathrm{SiO}_{2}$ and an index $=1.45$. Therefore for TM polarization, $\mathrm{n}_{1}$ and $\mathrm{n}_{2}$ are equal to 2.064343 and 1.45, respectively.

$$
\begin{gathered}
\frac{1}{n_{T M_{H}}^{2}}=\frac{f f_{H}}{2.064343^{2}}+\frac{1-f f_{H}}{1.45^{2}} \\
\frac{1}{n_{T M_{L}}^{2}}=\frac{f f_{L}}{2.064343^{2}}+\frac{1-f f_{L}}{1.45^{2}} \\
n_{\text {avg }_{e f f}^{T M}}=f f \cdot n_{T M_{H}}+(1-f f) \cdot n_{T M_{L}} \\
1.842617=f f \cdot\left(\frac{1}{\sqrt{\frac{f f_{H}}{2.064343^{2}}+\frac{1-f f_{H}}{1.45^{2}}}}\right)+ \\
(1-f f) \cdot \frac{1}{\sqrt{\frac{f f_{L}}{2.064343^{2}}+\frac{1-f f_{L}}{1.45^{2}}}}
\end{gathered}
$$


On Solving equation (5.8), with $\mathrm{ff}=0.5$, for $\mathrm{ff}_{\mathrm{L}}$ and $\mathrm{ff}_{\mathrm{H}}$, we attain equation (5.9). This equation can be satisfied for multiple values of $\mathrm{ff}_{\mathrm{L}}$ and $\mathrm{ff}_{\mathrm{H}}$, plotted in Figure 5.17 .

$$
\sqrt{\frac{1}{1.97383-\mathrm{ff}_{\mathrm{L}}}}+\sqrt{\frac{1}{1.97383-\mathrm{ff}_{\mathrm{H}}}}=1.80902
$$

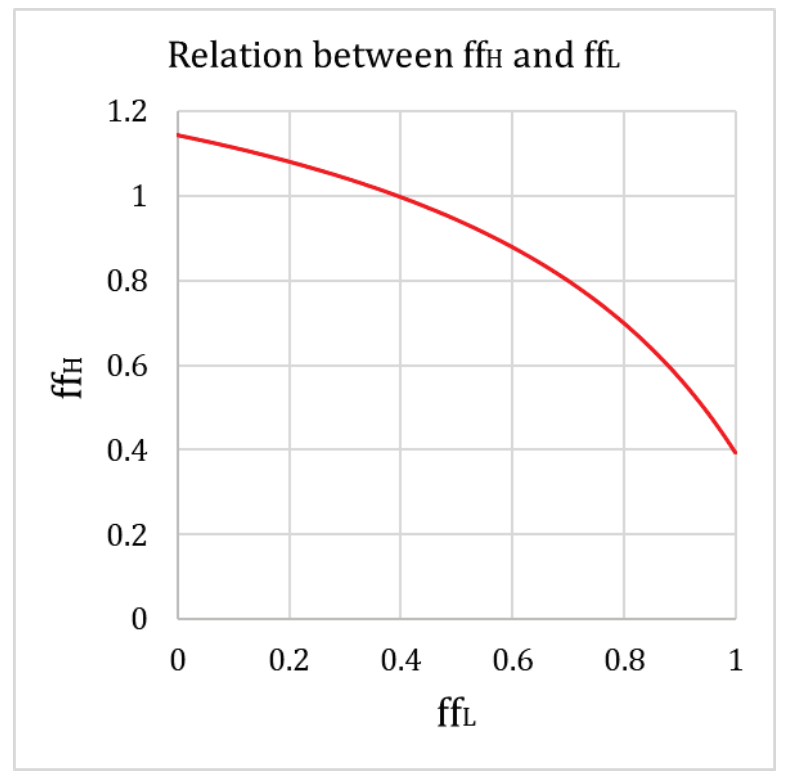

Figure 5.17. Plot for equation (5.9)

Assuming the high index region is unetched silicon block, therefore for $\mathrm{ff}_{\mathrm{H}}=$ 1.0 , the value for $\mathrm{ff}_{\mathrm{L}}$ which satisfies the above equation is 0.3943 , resulting in the initial sub-wavelength grating coupler design described in Table 5-5. The partial etched low index region of the grating coupler was replaced by full-etched subwavelength structures (Figure 5.18). The number of sub-wavelength structures in the high and the low index regions are referred to as periods and are denoted by $N_{\mathrm{H}}$ and $N_{\mathrm{L}}$. The number of periods in the low-index region is varied to achieve 
maximum coupling efficiency as well as bandwidth for both the polarizations at the central wavelength of $1.55 \mu \mathrm{m}$.

Table 5-5 Initial sub-wavelength grating design

\begin{tabular}{|l|l|l|l|l|l|}
\hline $\boldsymbol{\Lambda}(\mathbf{n m})$ & $\mathbf{f f}$ & \multicolumn{1}{|c|}{$\mathbf{f f}_{\mathbf{L}}$} & \multicolumn{1}{|c|}{$\mathbf{f f}_{\mathbf{H}}$} & \multicolumn{1}{|c|}{$\boldsymbol{N}_{\mathbf{H}}$} & \multicolumn{1}{|c|}{$\boldsymbol{N}_{\mathbf{L}}$} \\
\hline 675 & 0.5 & 0.3943 & 1.0 & 1 & 2 \\
\hline
\end{tabular}

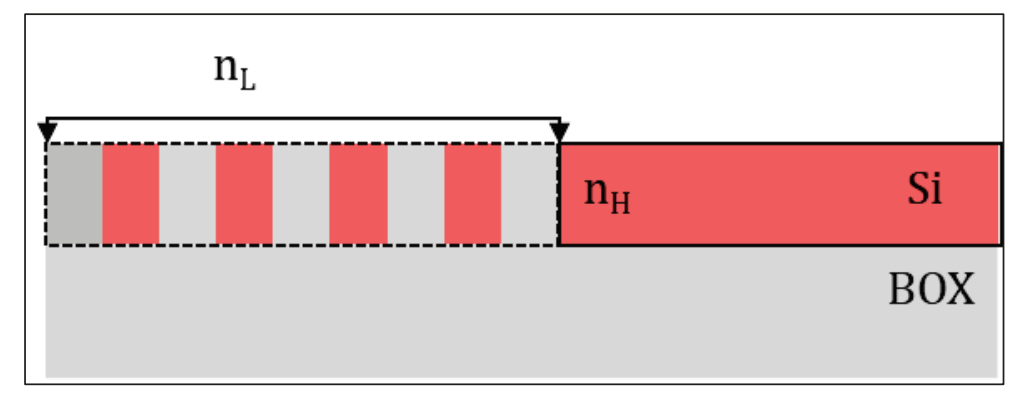

Figure 5.18. Sub-wavelength structure in high and low index regions

For the initial SWGC design, the low-index region is constituted by two subwavelength structures such that the length occupied by them results in $\mathrm{ff}_{\mathrm{L}}=0.3943$. The resultant transmission spectrum of the initial SWGC design is illustrated in Figure 5.19. We observe that both TE and TM polarized mode are couple in but with a drop in the maximum coupling efficiency by almost $50 \%$ for both polarizations. 


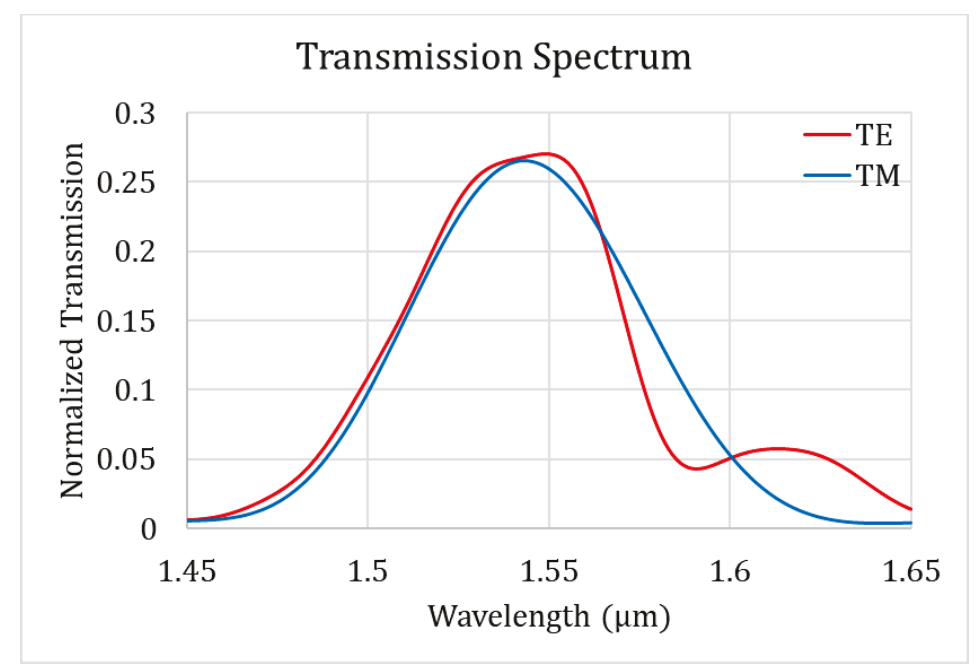

Figure 5.19. Transmission spectrum for the initial SWGC

To further increase the bandwidth and the CE of the grating coupler for both TE and TM polarization the grating period must be increased as per the bandwidth analysis in Section 3.1.5 . Additionally, the sub-wavelength grating parameters must be optimized to achieve maximum coupling efficiency at $1550 \mathrm{~nm}$. The parameters that were optimized are - (1) Grating period $(\Lambda)(2)$ duty cycle of the grating $\left(f f_{1}\right)$ (3) the fill-factor of the low-index region, $\left(f f_{L}\right)(4)$ the number of periods in the low index region, $N_{L}$.

\subsubsection{Parametric Sweeps for SWGC}

\section{A. Grating Period}

We know from bandwidth analysis in Section 3.1.5, that an increase in the grating period results in an increase in the coupling bandwidth by reducing the effective index of the grating. To prove this, for constant duty-cycle $\left(\mathrm{ff}_{1}=0.5\right)$, lowindex fill-factor $\left(\mathrm{ff}_{\mathrm{L}}=0.39\right)$, high-index fill-factor $\left(\mathrm{ff}_{\mathrm{H}}=1.0\right)$ and number of periods in the low index region $\left(\mathrm{n}_{\mathrm{L}}=2\right)$, the transmission spectrum for TE and TM polarizations 
were observed for varying grating periods. Figure 5.20 and Figure 5.21 illustrate the transmission spectrum for TE and TM polarization, respectively for various grating periods ranging from $675 \mathrm{~nm}-1200 \mathrm{~nm}$. For grating period > 1300nm, the average transmission for TE polarized light was insignificant.

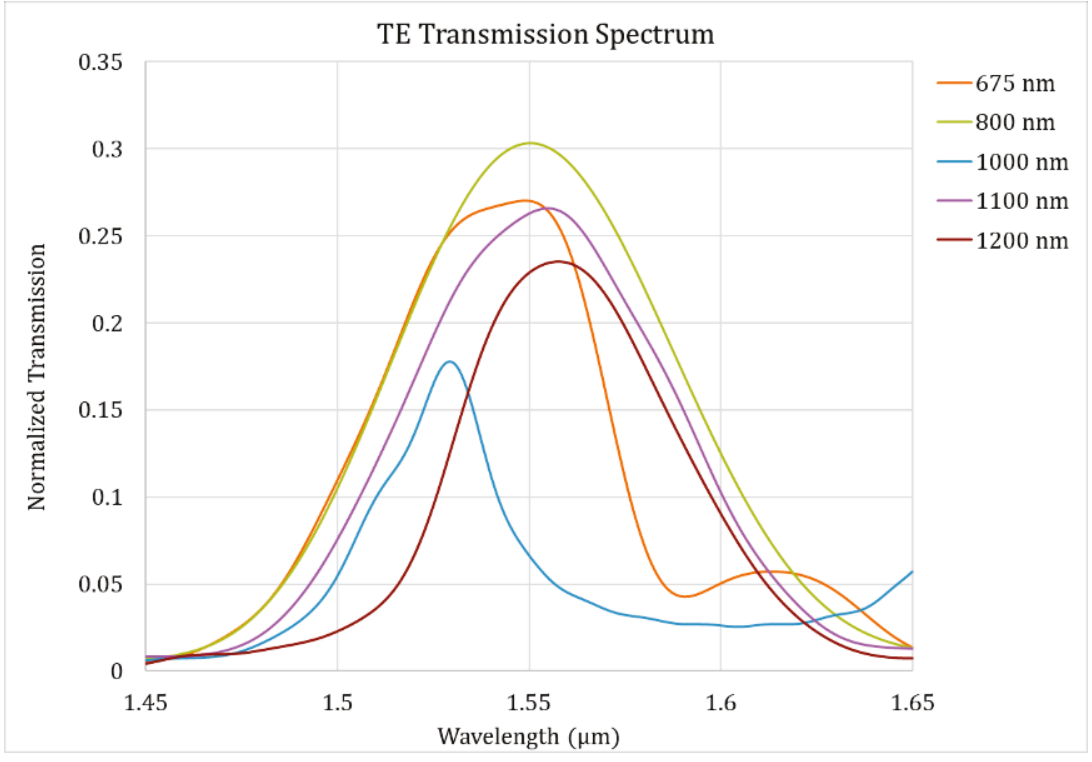

Figure 5.20. Variation of transmission spectrum with respect to grating period of SWGC for TE polarization

For TE polarization, the maximum coupling is observed for grating period $800 \mathrm{~nm}$ and with an increase in grating period the coupling efficiency and bandwidth both reduced. Whereas, for the TM polarized mode the coupling bandwidth increase at the expense of the coupling efficiency for grating period ranging from 800 - $1200 \mathrm{~nm}$. While the lowest bandwidth and transmission was observed for $\Lambda=675$ nm. 


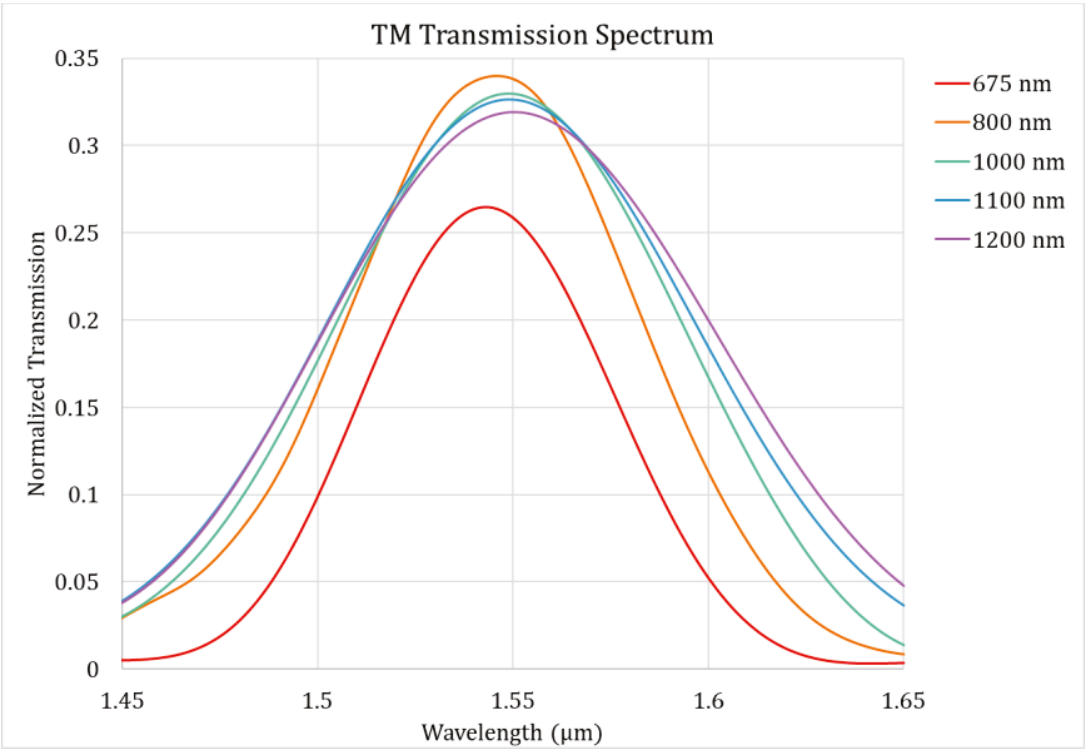

Figure 5.21. Variation of transmission spectrum with respect to grating period of SWGC for TM polarization

\section{B. Duty-Cycle of the Grating}

The average effective index of the grating coupler is directly proportional to the duty-cycle/fill-factor of the grating coupler, as evident from equation (5.4). In this section, we observe the effect of the duty cycle on the SWGC performance. The transmission spectrum for the TE and the TM polarizations for varying duty-cycle is depicted in Figure 5.22 and Figure 5.23, respectively. For constant grating period $(\Lambda=$ $800 \mathrm{~nm})$, low-index region fill-factor $\left(\mathrm{ff}_{\mathrm{L}}=0.39\right)$, high-index region fill-factor $\left(\mathrm{ff}_{\mathrm{H}}=1\right)$, the duty-cycle, ff was varied from $20 \%$ to $80 \%$. For TE and TM polarized incident mode, maximum transmission and coupling bandwidth at central wavelength of 1.55 $\mu \mathrm{m}$ was observed for $f f_{1}=0.6$. At duty-cycle, $f f_{1}=0.5$, a slightly lower maximum transmission and a lower coupling bandwidth for both TE and TM polarization. The maximum transmission of TM was observed to be more than TE at all values of $\mathrm{ff}_{1}$. 
We therefore, select our range for the duty-cycle $\left(\mathrm{ff}_{1}\right)$ as $50 \%$ to $60 \%$ for further optimization, to obtain the maximum possible average transmission for both polarizations.

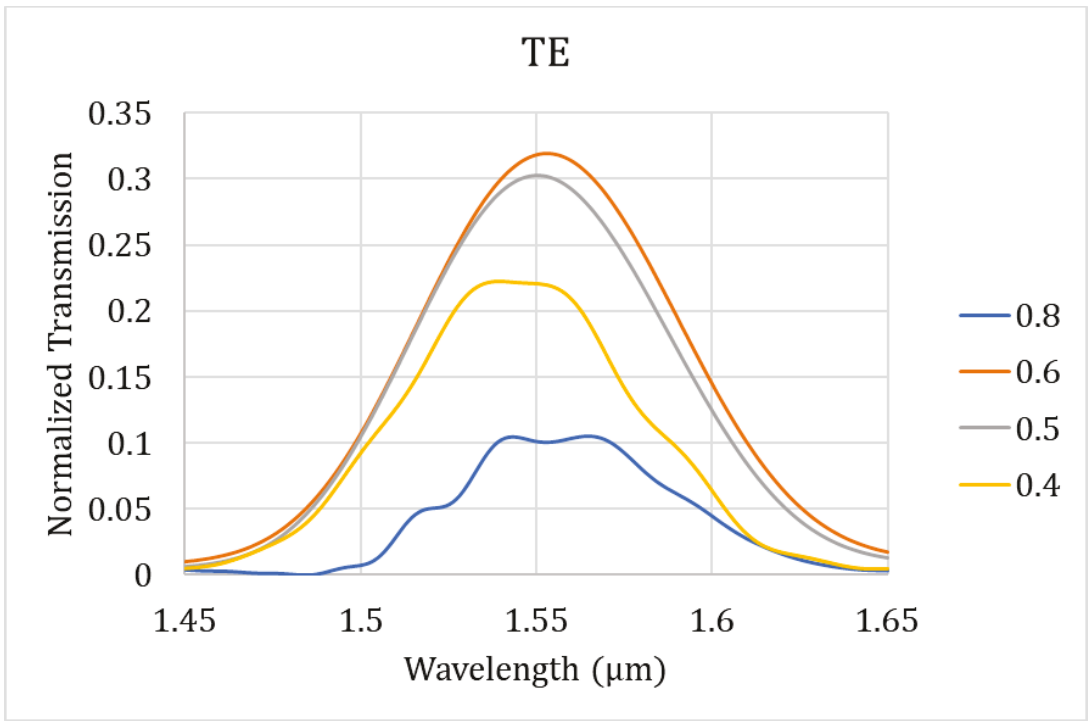

Figure 5.22. Variation of transmission spectrum for TE polarization with duty cycle of the grating period

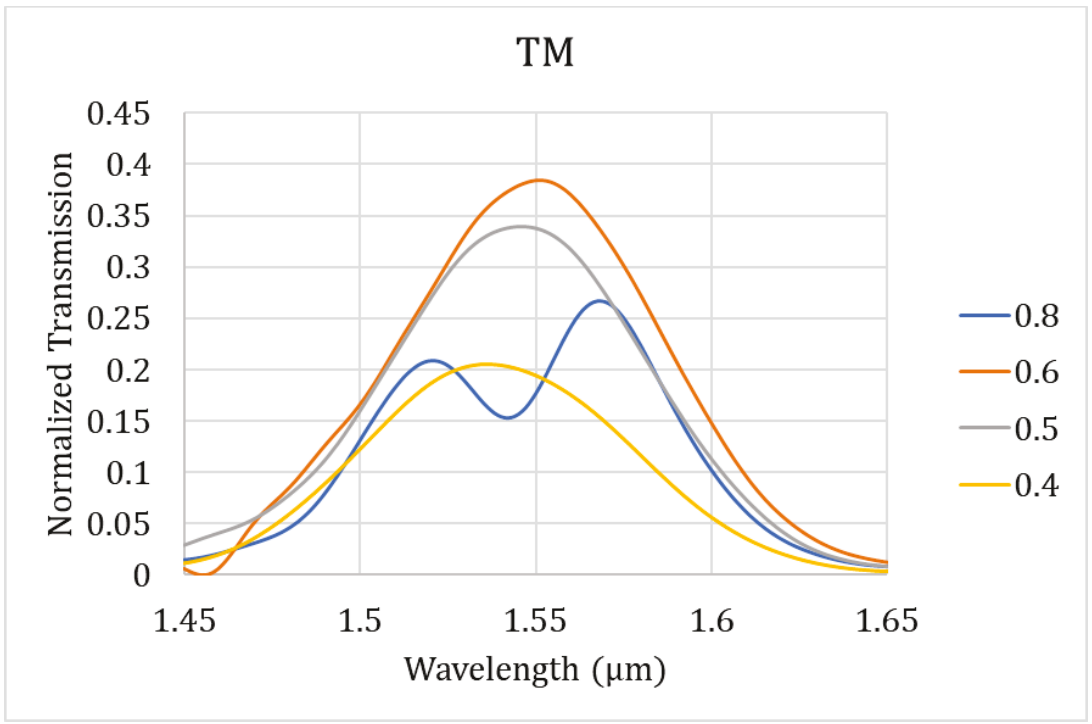

Figure 5.23. Variation of transmission spectrum for TM polarization with duty cycle of the grating period 


\section{Fill-Factor of low index region}

The fill-factor $\left(\mathrm{ff}_{\mathrm{L}}\right)$ governs the effective index of the low-index region by Rytov's formula, equations (4.13) and (4.21). A larger fill-factor (ff.), results in higher effective index of the low-index region. This results in lower refractive index contrast between the high and the low-index regions of the grating, and a reduced grating strength. The variation of the transmission spectrum with respect to the fill-factor of the low index regions for TE and TM polarization is depicted in Figure 5.24 and Figure 5.25, respectively. For the constant grating parameters- grating period $(\Lambda=800$ $\mathrm{nm})$, duty-cycle ( $\left(\mathrm{ff}_{\mathrm{l}}=0.6\right)$, high-index region fill-factor $\left(\mathrm{ff}_{\mathrm{H}}=1.0\right)$, number of periods in low-index region $\left(\mathrm{N}_{\mathrm{H}}=2\right)$ and low-index region fill-factor varying from $20 \%-80 \%$, the transmission spectrum as observed for both TE and TM polarizations are depicted in Figure 5.24 and Figure 5.25, respectively. Maximum transmission and coupling bandwidth is observed for $\mathrm{ff}_{\mathrm{L}}=20 \%$ for both TE and TM polarization, with low PDL. For further bandwidth and coupling efficiency optimization, we restrict the $\mathrm{ff}_{\mathrm{L}}$ to $\sim 20 \%$. 


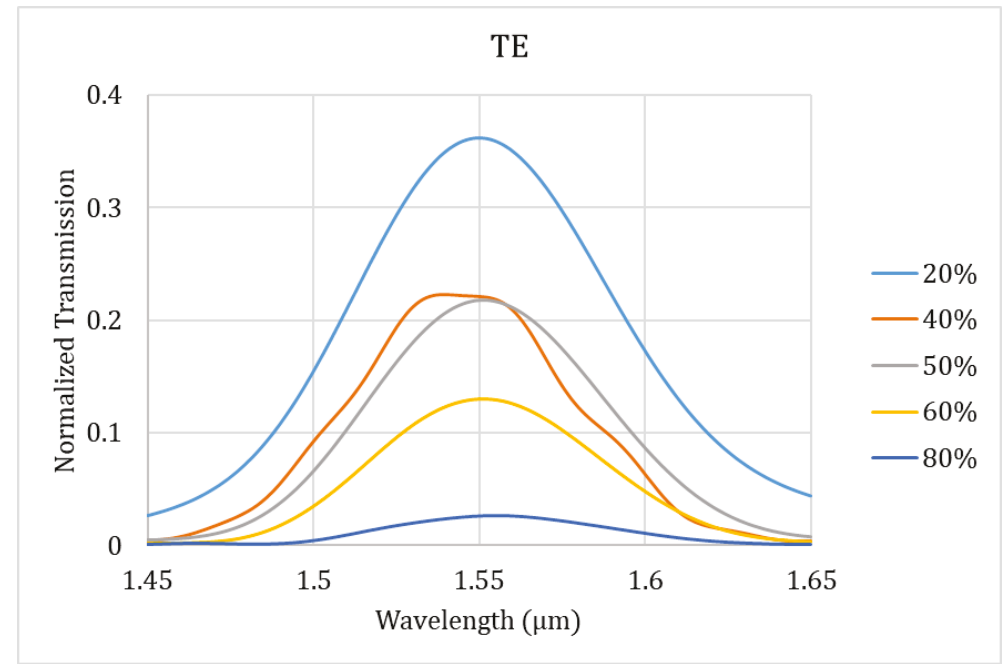

Figure 5.24. Variation of the transmission spectrum with fill-factor (ffL) for TE polarized mode

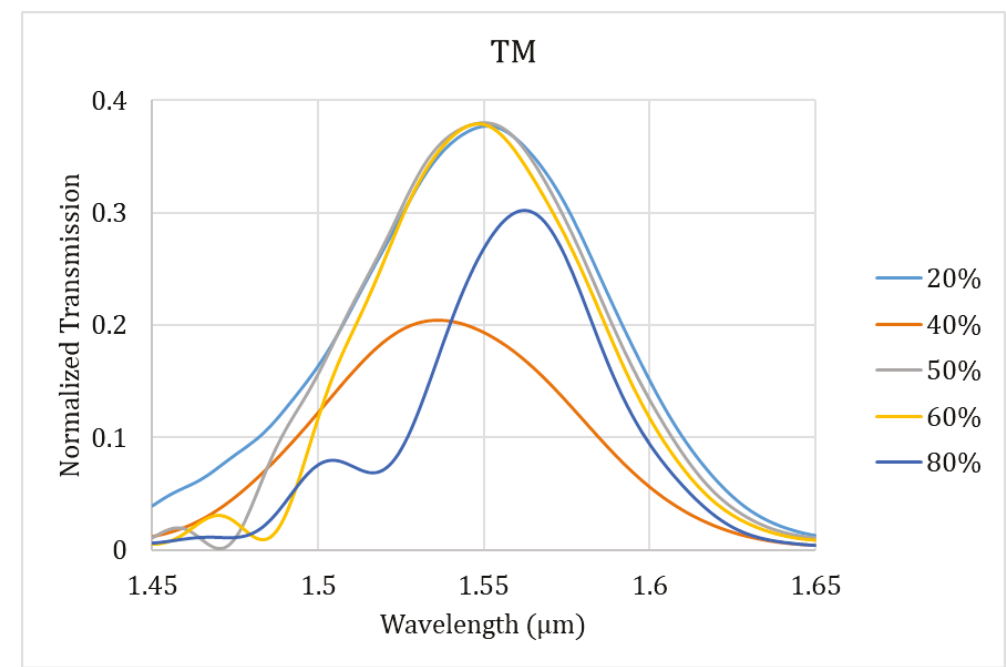

Figure 5.25. Variation of the transmission spectrum with fill-factor (ffL) for TM polarized mode 


\section{Number of periods in the low-index region}

The effect of the number of periods in the low index region is more profound for the TE polarization than TM. For the constant grating parameters- grating period $(\Lambda=800 \mathrm{~nm})$, duty-cycle $(\mathrm{ff}=0.6)$, high-index region fill-factor $(\mathrm{ff}=1.0)$, low-index region fill-factor $\left(\mathrm{ff}_{\mathrm{L}}=0.2\right)$ and varying $\mathrm{N}_{\mathrm{L}}$, the transmission spectrum approximately remains constant and notices negligible change for TM on varying the number of periods, $\mathrm{N}_{\mathrm{L}}$ from 1 to 5 . Whereas for TE, we see a $5 \%$ increment in the transmission for $\mathrm{N}_{\mathrm{L}}=1$ and $\mathrm{N}_{\mathrm{L}}=3,5$.

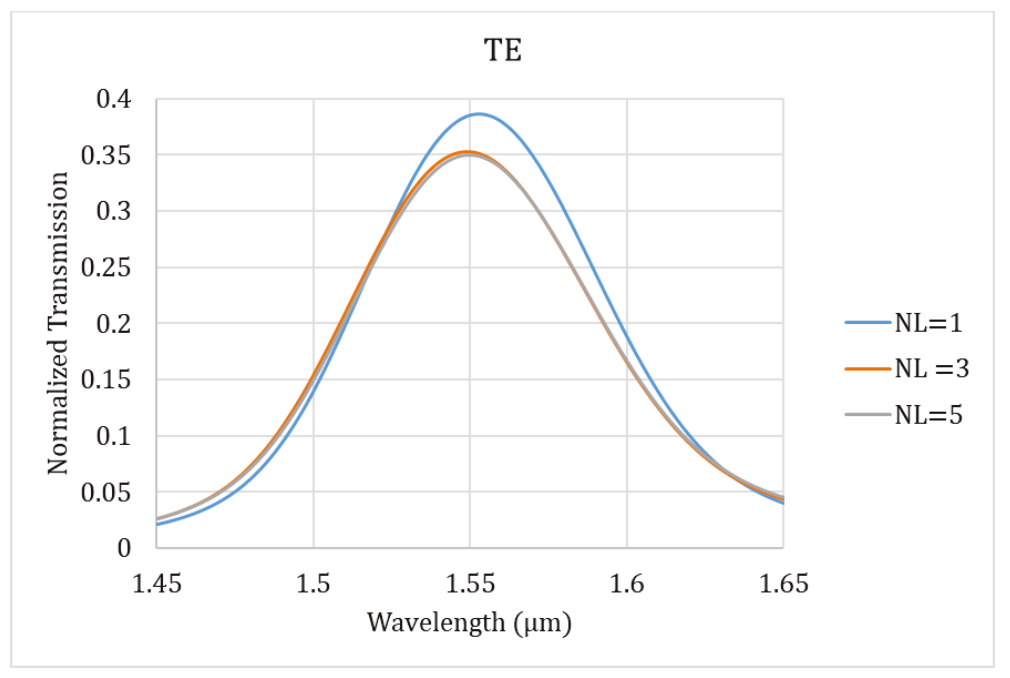

Figure 5.26. Variation of Transmission spectrum with number of periods $\left(\mathrm{N}_{\mathrm{L}}\right)$ in the low index region for TE polarization 


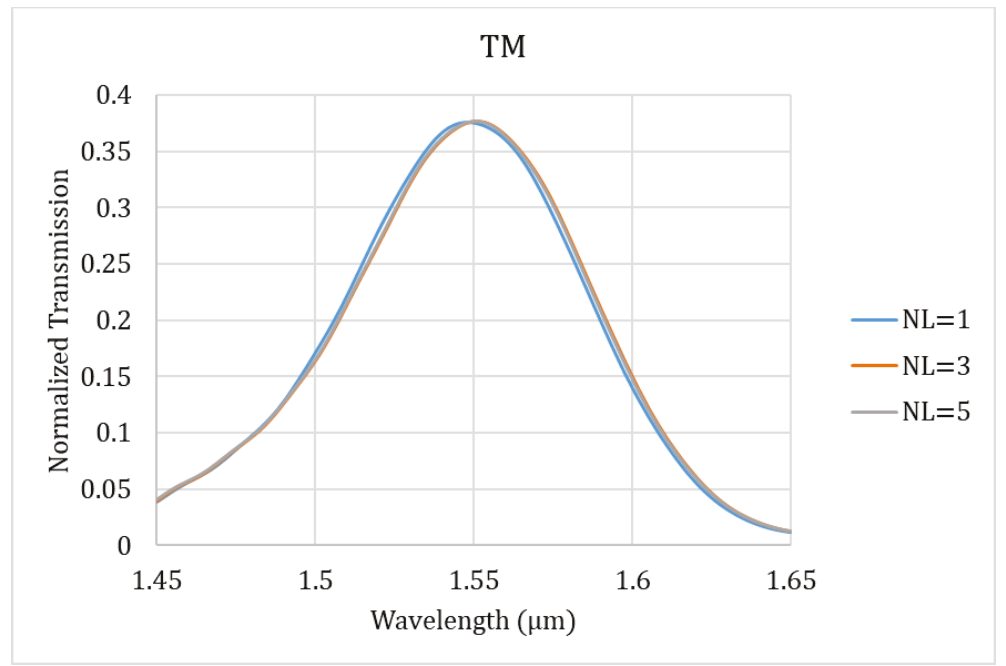

Figure 5.27. Variation of the Transmission spectrum with number of periods $\left(\mathrm{N}_{\mathrm{L}}\right)$ in the low index region for TM polarization 


\subsubsection{Simulation Results of Polarization Flexible and Broadband SWGC}

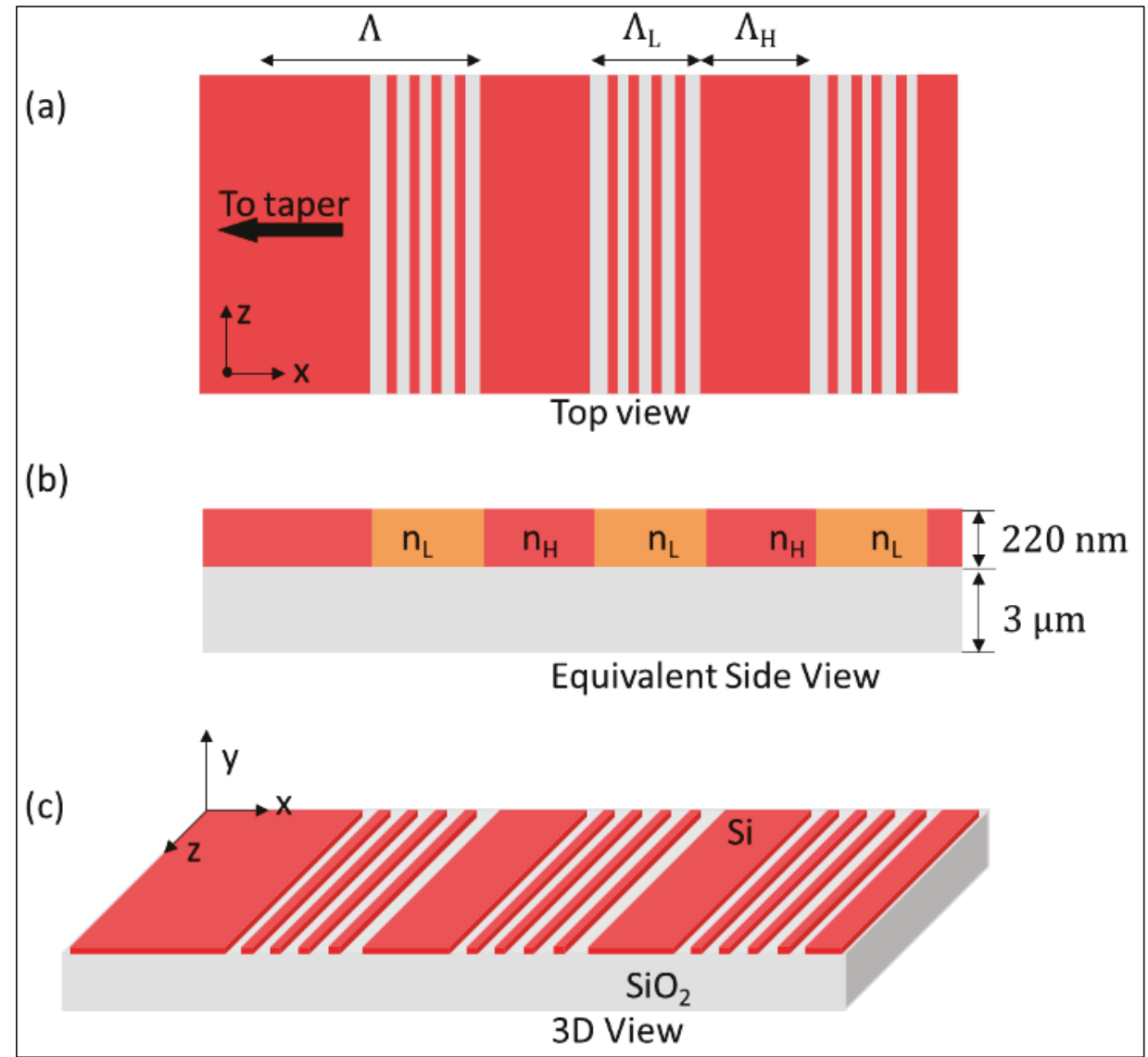

Figure 5.28. Schematic diagrams for the sub-wavelength grating coupler (SWGC) (a) Top View (b) Cross-section view with effective index approximation (c) 3D representation.

The schematic diagram of the final the broadband and polarization flexible sub-wavelength grating coupler is illustrated in Figure 5.28. The sub-wavelength grating coupler (SWGC) consists of alternating high and low index regions as seen in Figure 5.28 (b). A top-view of the SWGC is shown in Figure 5.28 (a), where the grating period is denoted by $\Lambda$. The length of the high-index regions and the low- 
index region are $\Lambda_{\mathrm{H}}$ and $\Lambda_{\mathrm{L}}$, respectively, such that $\Lambda_{\mathrm{L}}+\Lambda_{\mathrm{H}}=\Lambda$. The duty cycle or the fill-factor, denoted by $f f=\frac{\Lambda_{H}}{\Lambda}$, is described as the ratio of the length of highindex region to the total grating period. The high index region is made up of one single silicon segment $\left(f f_{H}=1.0\right)$ with an effective index of 2.744 at $1.55 \mu \mathrm{m}$ for the $\mathrm{TE}_{0}$ polarization and the index of the low-index region is engineered using SWGs. The low-index region has periodic regions of $\mathrm{Si}$ and $\mathrm{SiO}_{2}$ with a fill-factor of $f f_{L}$. The fill-factor of low index region is calculated as the sum of the lengths of all the silicon SWG divided by $\Lambda_{\mathrm{L}}$. The approximate value of $n_{L}$ as calculated using Rytov's Formula for $T E_{0}$ equals 1.7855. Figure 5.28(c), illustrates the arrangement of the sub-wavelength gratings in a single pitch of the SWGC. In this design we used uniform duty cycles across the entire grating length. The front-view of the SWGC for 2D-FDTD simulation is depicted in Figure 5.29 below. Appendix B contains the Lumerical scripts for generation of SWGC structure for 2D-FDTD Simulation. Appendix $\mathbf{C}$ contains the model setup and model analysis scripts for analyzing the results. 


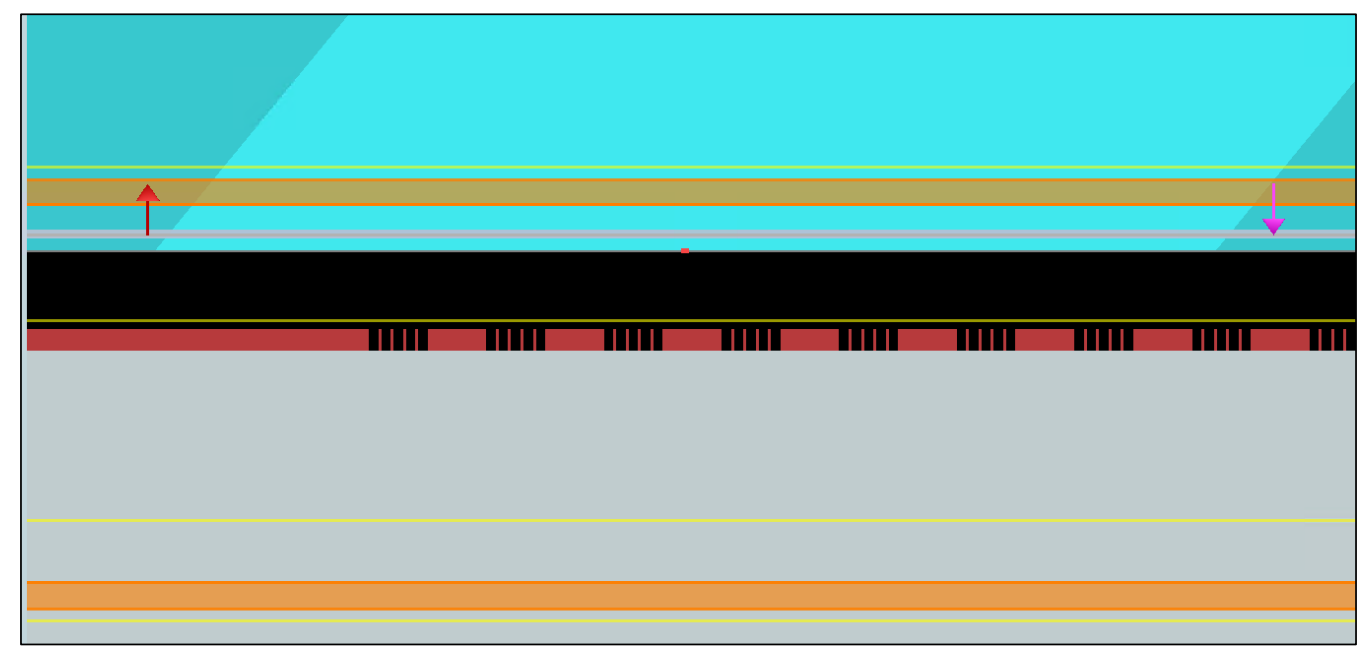

Figure 5.29. Simulation setup for a SWGC

Optimizations from previous section, for both the polarizations resulted in the final device parameters listed in Table 5-6, where both the $\mathrm{TE}_{0}$ and $\mathrm{TM}_{0}$ polarizations are co-propagating in the same silicon waveguide. The optimized fill factor is $f f=0.5$ with the high and low index region having duty cycle of $f f_{L}=$ 0.2 and $f f_{H}=1.0$ respectively. For this design the number of $\mathrm{SWG}$ periods in the low index region were optimized to $N_{L}=4$, resulting in the smallest feature size of Si to be $29.375 \mathrm{~nm}$. It is noted that, although the designed grating coupler is identical for both polarizations, the fiber is set to incident at an angle of $39^{\circ}$ and $16^{\circ}$ for the $\mathrm{TE}_{0}$ and $\mathrm{TM}_{0}$, respectively, to obtain maximum coupling efficiency.

Table 5-6. Optimized design parameters for the polarization flexible and broadband grating coupler design

\begin{tabular}{|l|l|l|l|l|l|l|l|}
\hline $\boldsymbol{\Lambda}(\mathbf{n m})$ & $\mathbf{f f}$ & $\mathbf{f f}_{\mathbf{L}}$ & \multicolumn{1}{|c|}{$\mathbf{f f}_{\mathbf{H}}$} & $\boldsymbol{N}_{\mathbf{H}}$ & $\boldsymbol{N}_{\mathbf{L}}$ & \multicolumn{2}{|c|}{$\boldsymbol{\theta}$} \\
\hline $\mathbf{1 1 7 5}$ & 0.5 & 0.2 & 1.0 & 1 & 4 & $\mathrm{TE}=39^{\circ}$ & $\mathrm{TM}=16^{\circ}$ \\
\hline
\end{tabular}


Table 5-7. Simulation results for polarization flexible and broadband grating design using 2D -FDTD

\begin{tabular}{|l|l|l|l|l|}
\hline Polarization & \multicolumn{2}{|l|}{ Coupling Efficiency (dB) } & \multicolumn{2}{l|}{ Bandwidth (nm) } \\
\cline { 2 - 5 } & Maximum & @1550 nm & 1-dB & 3-dB \\
\hline TE & $-4.86 @ 1554 \mathrm{~nm}$ & -4.88 & 57.73 & 105 \\
\hline TM & $-4.88 @ 1549 \mathrm{~nm}$ & -4.88 & 69.72 & 121 \\
\hline
\end{tabular}

Table 5-8 Simulation results for polarization flexible and broadband grating design using 3D- FDTD

\begin{tabular}{|l|l|l|l|l|}
\hline Polarization & \multicolumn{2}{|l|}{ Coupling Efficiency (dB) } & \multicolumn{2}{l|}{ Bandwidth (nm) } \\
\cline { 2 - 5 } & Maximum & @1550 nm & 1-dB & 3-dB \\
\hline TE & $-5.45 @ 1553 . \mathrm{nm}$ & -5.20 & 62.7 & 106.17 \\
\hline TM & & & & 117.27 \\
\hline
\end{tabular}

The simulation results with the 2D and 3D FDTD methods are depicted in Table 5-7and Table 5-8, respectively. With the optimized grating parameters with 2DFDTD simulations resulted in a maximum coupling efficiency of $-4.86 \mathrm{~dB}$ and -4.88 $\mathrm{dB}$ for TE and TM polarizations, respectively. The polarization dependent losses were reduced to zero at $1.55 \mu \mathrm{m}$ since both TE and TM polarized light exhibited a coupling efficiency of $-4.88 \mathrm{~dB}$. The 1 -dB bandwidth of $57.73 \mathrm{~nm}$ was obtained for TE polarized light, whereas TM polarized light exhibited a 1-dB coupling bandwidth 
of $69.72 \mathrm{~nm}$. While the $3-\mathrm{dB}$ bandwidth of $105 \mathrm{~nm}$ was achieved for the TE polarization, the TM polarization attained $121 \mathrm{~nm}$ of coupling bandwidth. The 2DFDTD transmission spectrum of the SWGC is shown in Figure 5.30. A maximum transmission of $\sim 32.65 \%$ can be observed from the transmission spectrum below. The coupling efficiencies for the 2D FDTD SWGC is given in Figure 5.31. The coupling peaks of TE and TM vary by a meagre $5 \mathrm{~nm}$, which is well within the tolerance limit. On the other hand, the 3D- FDTD depicts a reduction of maximum CE for TE by almost $0.6 \mathrm{~dB}$ and $0.3 \mathrm{~dB}$ for TM. These differences can be attributed to the losses incurred by the tapers in the focussing grating structure, since these are not $100 \%$ adiabatic. The $1-\mathrm{dB}$ and the $3-\mathrm{dB}$ bandwidths for both TE and TM remain the equivalent, with TE exhibiting a slightly improved 1-dB bandwidth of $62 \mathrm{~nm}$. The PDL observed with 3D FDTD simulations at $1550 \mathrm{~nm}$ are increased to $0.26 \mathrm{~dB}$ along with a slightly higher coupling peak difference of $8 \mathrm{~nm}$. The coupling efficiencies attained through 3D FDTD simulations are depicted in Figure 5.33

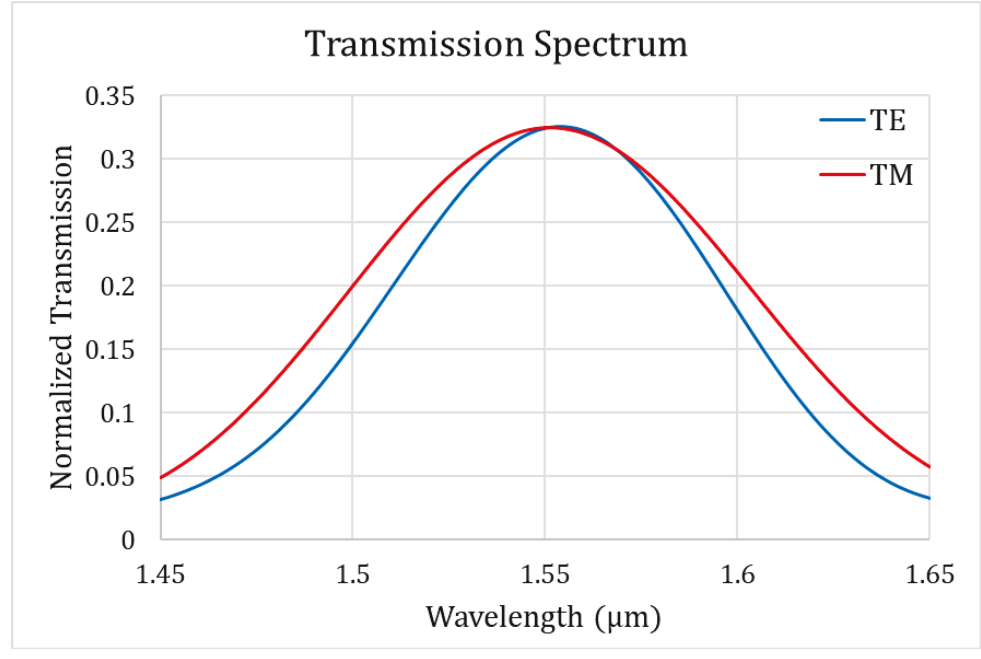

Figure 5.30. Normalized transmission spectrum for polarization flexible and broadband grating coupler 


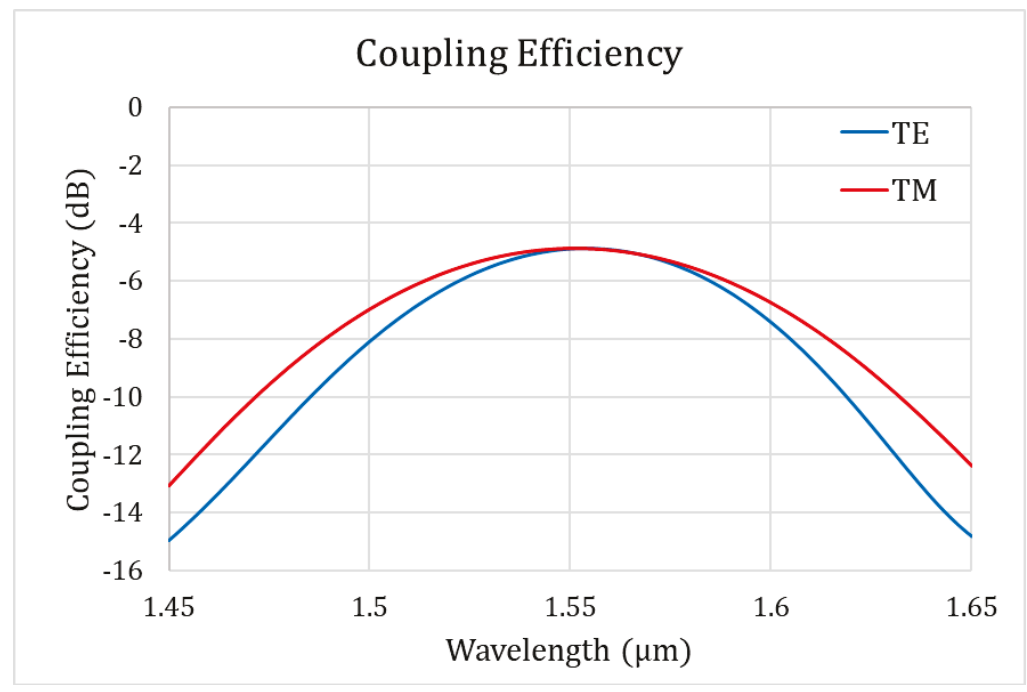

Figure 5.31. Coupling efficiency of the polarization flexible and broadband grating coupler
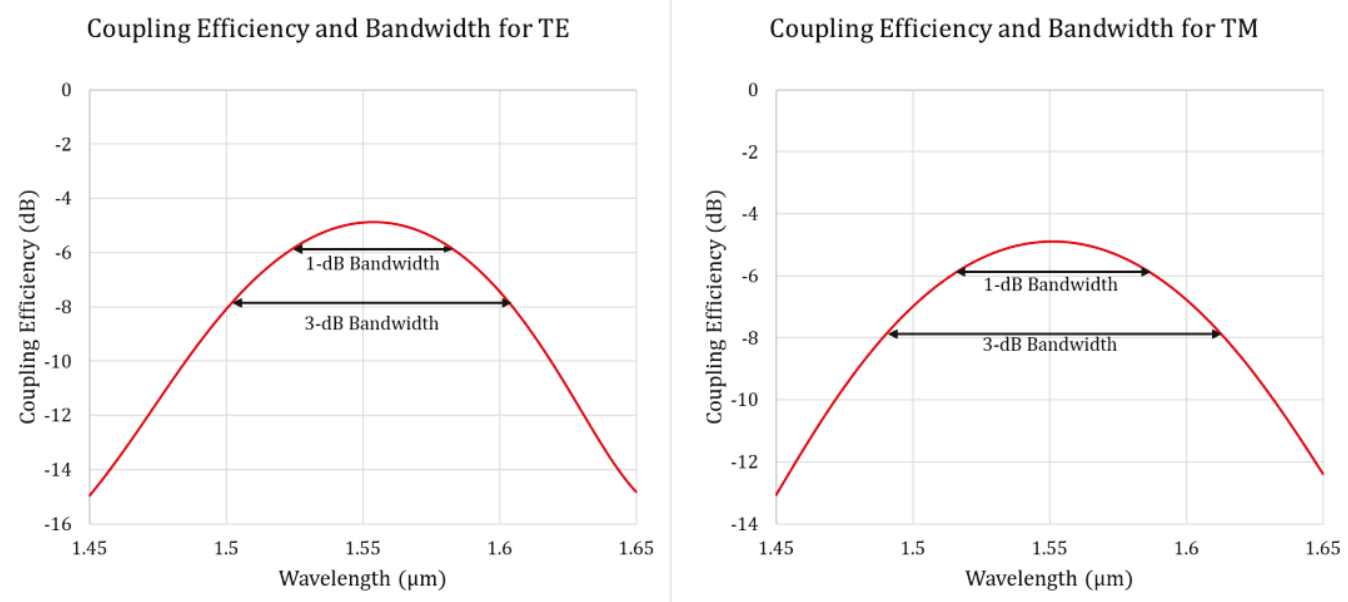

Figure 5.32. Coupling bandwidths for the polarization flexible and broadband grating 


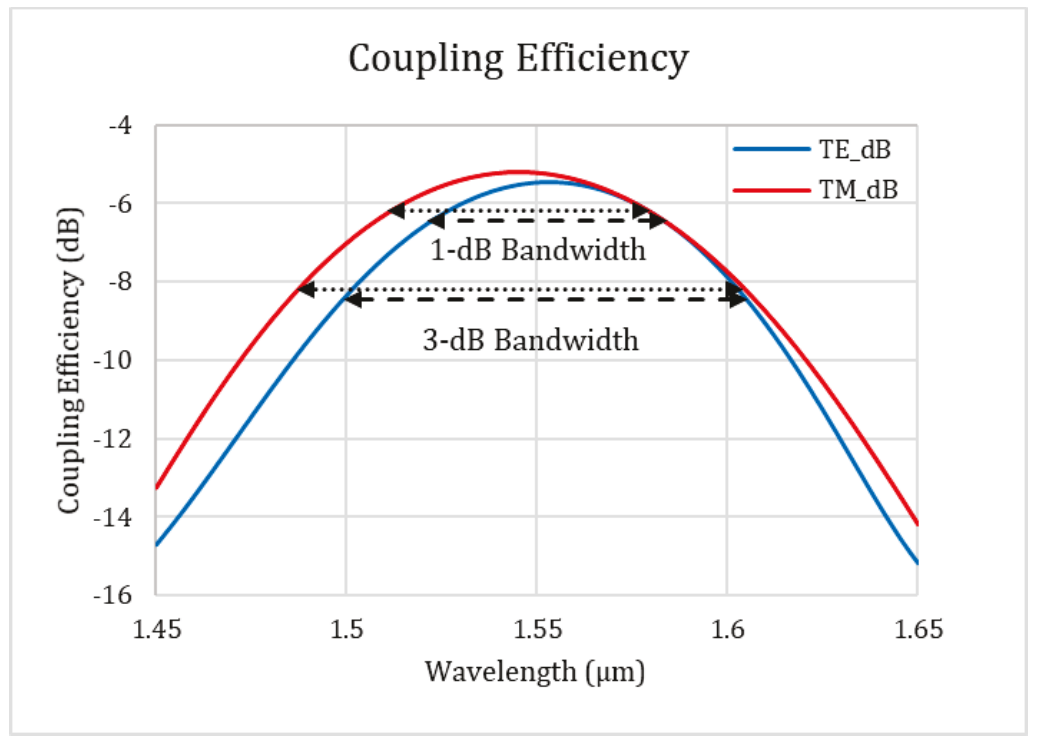

Figure 5.33. Coupling efficiency of the polarization flexible and broadband grating coupler using 3D- FDTD Simulation

It can be easily observed from the transmission spectrum that the coupling bandwidths of TM is greater than TE, which can be reasoned back to the Bandwidth Analysis of the grating coupler in Section 3.1.5 . The bandwidth of the two polarizations can be attributed to the equation- $\Delta \lambda_{1 d B}=2 \cdot \eta_{1 d B}\left|\frac{\left(-\Lambda \cdot n_{c} \cos \theta\right)}{\left(1-\Lambda \cdot \frac{d n_{e f f}(\lambda)}{d \lambda}\right)}\right|$. The bandwidth is dependent on the grating as well as the fiber parameters. The only two differentiating factors for the bandwidths of TE and TM are the incident angles and the dispersion. We know that bandwidth is directly proportional to the cosine of the angle at which the light must be incident, therefore $\cos \theta_{T E}<\cos \theta_{T M}$. The ratio of the two bandwidths, $\frac{3-d B \text { Bandwidth }_{T M}}{3-d B \text { Bandwidth }_{T E}}=\frac{121}{105}=1.1523$, whereas the $\frac{\cos \theta_{T M}}{\cos \theta_{T E}}=$ $\frac{0.9612}{0.7771}=1.23$, which is very close to the observed bandwidths. The 3-dB bandwidth of $\mathrm{TE}=105 \mathrm{~nm}$, whereas the $3-\mathrm{dB}$ bandwidth for $\mathrm{TM}=121 \mathrm{~nm}$. Therefore, $3-d B$ Bandwidth $_{T M} \approx 1.15 \times 3-d B$ Bandwidth $_{T E}$. The other factor 
contributing to the difference in the bandwidth is the dispersion term, $\frac{d n_{e f f}(\lambda)}{d \lambda}$. For TM the magnitude of absolute dispersion is greater for TM $\left(-8.8112 \times 10^{-5}\right)$ than that for TE $\left(-6.3946 \times 10^{-5}\right)$, resulting in a larger denominator in the bandwidth equation. The dispersion factor opposes the effect caused by the angle of incidence and therefore, the bandwidth ratio of $\mathrm{TM}$ to $\mathrm{TE}$ is $<1.23$.

The variation in the transmission spectrum of the SWGC with the number of periods in the low index region is depicted in Figure 5.34.
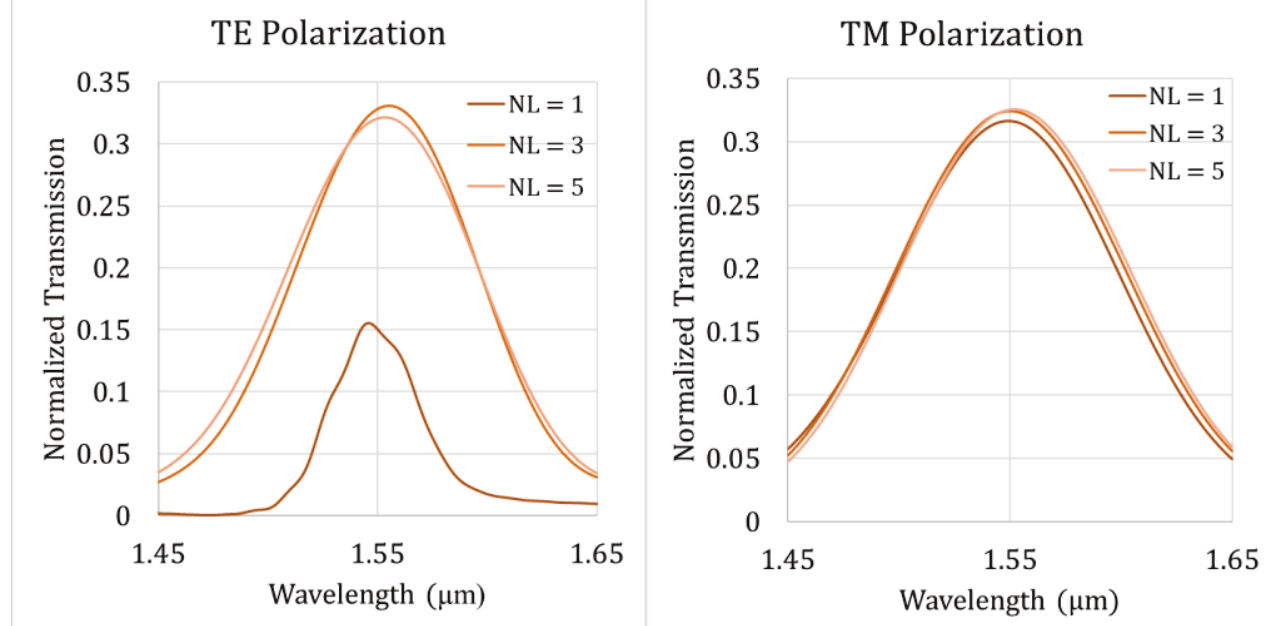

Figure 5.34. Variation of Transmission with respect to periods in the low index region

For TE polarization, it was observed that coupling bandwidth increased at the expense of the maximum coupling efficiency for $n_{\mathrm{L}}=5$ and $\mathrm{n}_{\mathrm{L}}=3$. For $\mathrm{n}_{\mathrm{L}}=1$, the average transmission is lower for TE, whereas for TM, the coupling efficiency remains mostly constant, increasing only slightly with the increase in the number of periods in the low-index region. The number of sub-wavelength structure a grating 
period was selected by considering the minimum feature size allowed for fabrication and was optimized for both TE and TM transmission for minimum polarization dependent losses at $1550 \mathrm{~nm}$.

To further optimize the results for the polarization flexible and broadband grating coupler, the Particle Swarm Optimization (PSO) from Lumerical was used, which used a large data space to converge to the global maxima based on a given FOM. The FOM, $C E_{1550 T E} \times B W_{3 d B T E}+C E_{1550 T M} \times B W_{3 d B T M}$, was maximized for this optimization. On analyzing the results obtained using PSO it was found that - 1) The grating coupler was optimized for only the TE polarized light, resulting in larger PDL losses, with TM mode depicting low coupling performance; 2) The optimized design parameters resulted in a grating coupler where the overall polarization independent/ flexible behavior was not satisfied. Therefore, application of PSO to optimize a polarization independent/ flexible design did not work as well as it would have for a polarization dependent grating design.

\subsubsection{S-Parameters of Polarization Flexible and Broadband SWGC}

S-Parameter for the TE and TM polarization are shown in Figure 5.35. It is seen that for both TE and TM polarization, $S_{12} \approx S_{21}$ which confirms with the Principle of Reciprocity. The back-reflections into the waveguide are given by $S_{22}$. The secondary vertical axis on the right denote the values for $S_{22}$. It is seen that for the TM polarization that the back reflections are as low as $-20 \mathrm{~dB}$ and $-10 \mathrm{~dB}$ for TE polarization at $1550 \mathrm{~nm}$. 

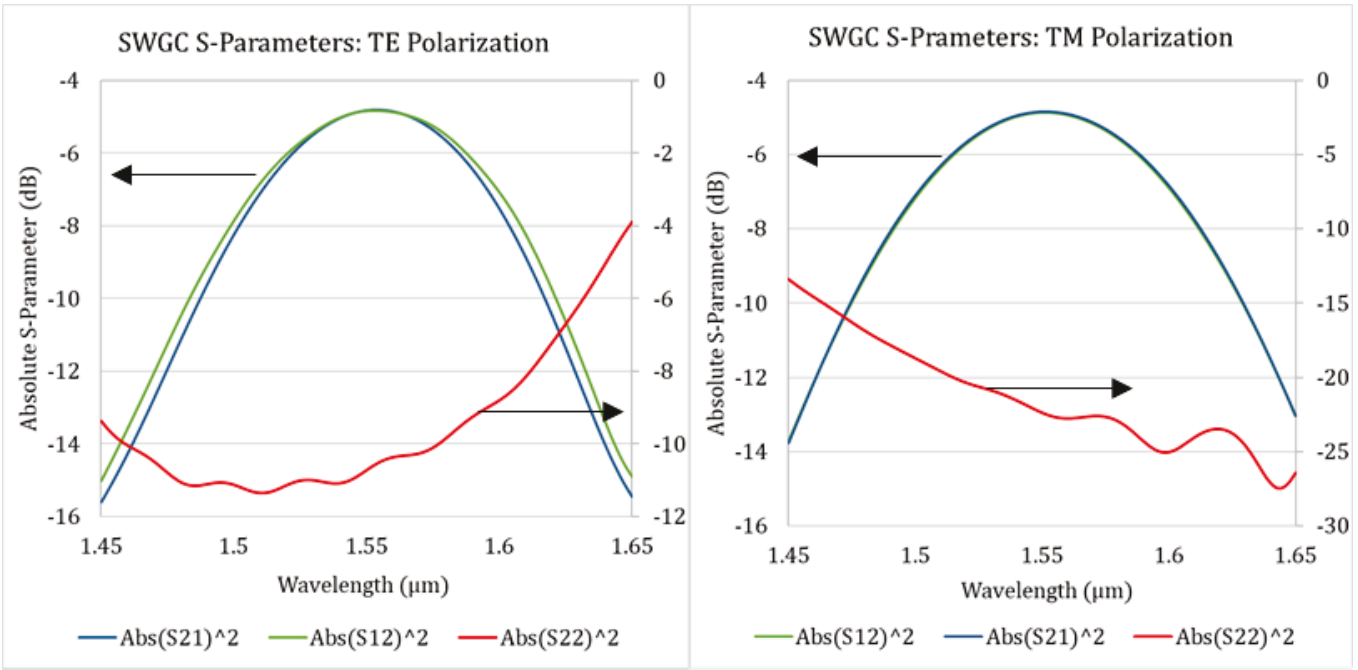

Figure 5.35. S-Parameter of the Polarization flexible and broadband SWGC

\subsection{Designing Apodized Sub-Wavelength Grating Coupler}

Fabrication of the polarization flexible and broadband SWGC in-house required a minimum feature size $>75 \mathrm{~nm}$. The design reported in the previous section had a minimum feature size of $29.375 \mathrm{~nm}$. Therefore, we modified the $\mathrm{SWGC}$ so that is well-suited for in-house fabrication. The design parameters and the performance metrics of the SWGC design for fabrication are listed in Table 5-9 and Table 5-10, respectively. The minimum feature size of this polarization flexible and broadband SWGC is $80.7812 \mathrm{~nm}$, i.e. each sub-wavelength structure in the low index region has a width of $80.7812 \mathrm{~nm}$. 
Table 5-9. Optimized design parameters for the fabrication of polarization flexible and broadband grating coupler design

\begin{tabular}{|l|l|l|l|l|l|l|}
\hline $\boldsymbol{\Lambda}(\mathbf{n m})$ & $\mathbf{f f}$ & $\mathbf{f f}_{\mathbf{L}}$ & \multicolumn{1}{|c|}{$\mathbf{f f}_{\mathbf{H}}$} & $\mathbf{n}_{\mathbf{H}}$ & $\mathbf{n}_{\mathbf{L}}$ & $\boldsymbol{\theta}$ \\
\hline $\mathbf{1 1 7 5}$ & 0.45 & 0.25 & 1.0 & 1 & 2 & $\begin{array}{l}\mathrm{TE}=39.176^{\circ} \\
\mathrm{TM}=15.5^{\circ}\end{array}$ \\
\hline
\end{tabular}

Table 5-10. Simulation results for design for fabrication of polarization flexible and broadband grating design

\begin{tabular}{|l|l|l|l|l|}
\hline Polarization & \multicolumn{2}{|l|}{ Coupling Efficiency (dB) } & \multicolumn{2}{l|}{ Bandwidth (nm) } \\
\cline { 2 - 5 } & Maximum & @1550 nm & 1-dB & 3-dB \\
\hline TE & $-4.88 @ 1550 \mathrm{~nm}$ & -4.88 & 47.29 & 69.5 \\
\hline TM & $-5.14 @ 1550 \mathrm{~nm}$ & -5.14 & 69.54 & 120.8 \\
\hline
\end{tabular}

It is evident from Table 5-10, the polarization dependent loss increased to $0.26 \mathrm{~dB}$ at $1550 \mathrm{~nm}$ for the SWGC designed for fabrication. Along with it, the coupling bandwidth of the TE polarized mode dropped by $30 \%$ and the maximum coupling efficiency of the TM polarization dropped below $-5 \mathrm{~dB}$. To improve the bandwidth of TE and CE of TM polarization, we introduced apodization to the SWGC design. We know from Section 3.3.1 that apodization alters grating strength along the grating, thereby increasing the overlap between the radiated beam and the gaussian beam of the fiber mode. The apodized polarization flexible and broadband SWGC performance is discussed in the following section. 


\subsubsection{Apodized Design for Fabrication}

We apodized the high-index region $\left(\mathrm{n}_{н}\right)$ for every consecutive grating period, thereby increasing the effective average index of the grating structure and the grating strength itself along the grating. The graphical design of the apodized grating structure is depicted in Figure 5.36.

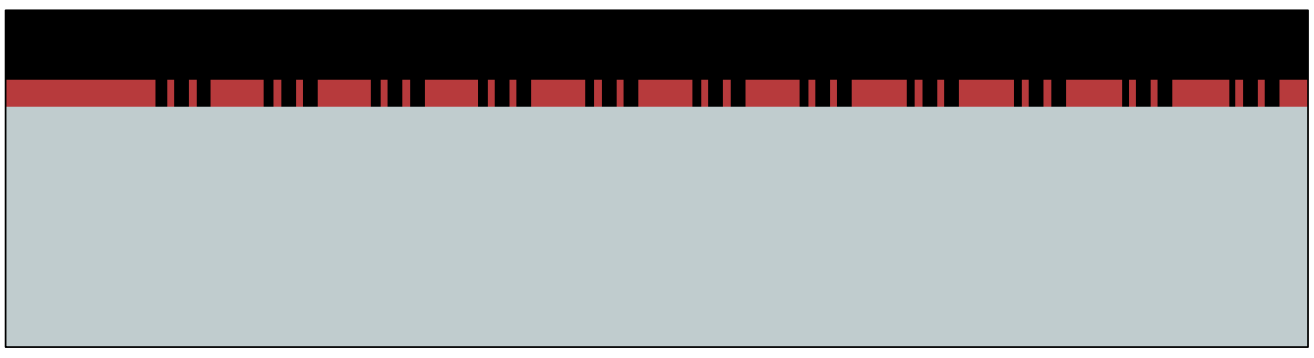

Figure 5.36. Apodized SWGC for polarization flexible and broadband operation

The size of the high-index region $\left(\mathrm{n}_{\mathrm{H}}\right)$ for each grating period are listed below-

\begin{tabular}{|c|c|c|c|}
\hline $\begin{array}{l}\text { Grating period } \\
\text { number }\end{array}$ & $\begin{array}{l}\text { Width of high-index } \\
\text { region, } \mathrm{n}_{\mathrm{H}}(\mu \mathrm{m})\end{array}$ & $\begin{array}{l}\text { Grating period } \\
\text { number }\end{array}$ & $\begin{array}{l}\text { Width of high- } \\
\text { index region, } \mathrm{n}_{\mathrm{H}} \\
(\mu \mathrm{m})\end{array}$ \\
\hline 1 & 0.568406 & 6 & 0.594844 \\
\hline 2 & 0.573694 & 7 & 0.600131 \\
\hline 3 & 0.578981 & 8 & 0.605419 \\
\hline 4 & 0.584269 & 9 & 0.610706 \\
\hline 5 & 0.589556 & 10 & 0.615994 \\
\hline
\end{tabular}

The design parameters of the apodized design are listed in Table 5-11. We observe an increase in the incidence angle for both TE and TM polarizations, which are well 
in accordance with the Bragg's condition, which suggests $\sin \theta \propto n_{\text {eff }}$. The simulation results of the apodized SWGC are presented in Table 5-12.

Table 5-11. Design parameters for broadband and polarization flexible apodized SWGC

\begin{tabular}{|l|l|l|l|l|l|l|c|}
\hline $\boldsymbol{\Lambda}$ & $\mathbf{f f}$ & $\mathbf{f f}_{\mathbf{L}}$ & $\mathbf{f f}_{\mathbf{H}}$ & $\mathbf{n}_{\mathbf{H}}$ & $\mathbf{n}_{\mathrm{L}}$ & Apodization & $\boldsymbol{\theta}$ \\
$\mathbf{( n m )}$ & & & & & & factor (apo) & \\
\hline 1175 & 0.45 & 0.25 & 1.0 & 1 & 2 & 1.009 & $\mathrm{TE}=44.88^{\circ}$ \\
& & & & & & & $\mathrm{TM}=17.17^{\circ}$ \\
\hline
\end{tabular}

Table 5-12. Simulation results for broadband and polarization flexible apodized SWGC

\begin{tabular}{|l|l|l|l|l|}
\hline Polarization & \multicolumn{2}{|l|}{ Coupling Efficiency (dB) } & \multicolumn{2}{l|}{ Bandwidth (nm) } \\
\cline { 2 - 5 } & Maximum & @1550 nm & 1-dB & 3-dB \\
\hline TE & $-4.78 @ 1550 \mathrm{~nm}$ & -4.78 & 51.45 & 88.66 \\
\hline TM & $-4.88 @ 1550 \mathrm{~nm}$ & -4.88 & 68.40 & 119.58 \\
\hline
\end{tabular}

We can see that by apodizing the grating, we were able to increase the maximum coupling efficiency for the TM polarization from $-5.14 \mathrm{~dB}$ back to $-4.88 \mathrm{~dB}$ and at the same time the 3-dB bandwidth for TE polarization increased from $69 \mathrm{~nm}$ to 88 nm. Therefore, apodization of the grating coupler resulted in an increased coupling bandwidth as well as the coupling efficiency. The transmission spectrum and the coupling efficiency of the apodized SWGC designed for fabrication is given in Figure 
5.37 and Figure 5.38, respectively. A PDL of $0.1 \mathrm{~dB}$ at $1550 \mathrm{~nm}$ can be observed from Figure 5.38.

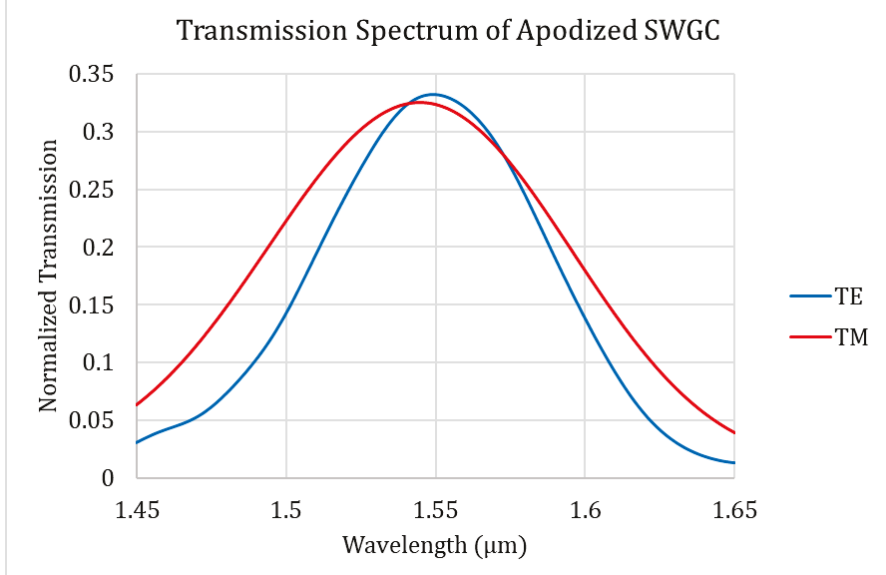

Figure 5.37. Transmission spectrum for the apodized SWGC

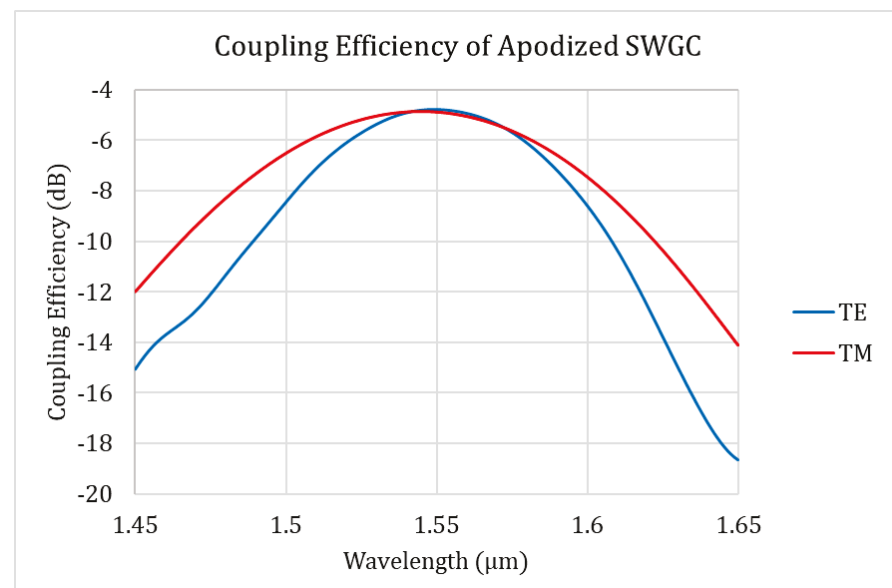

Figure 5.38. Coupling efficiency of the apodized SWGC 


\subsubsection{S-Parameters}
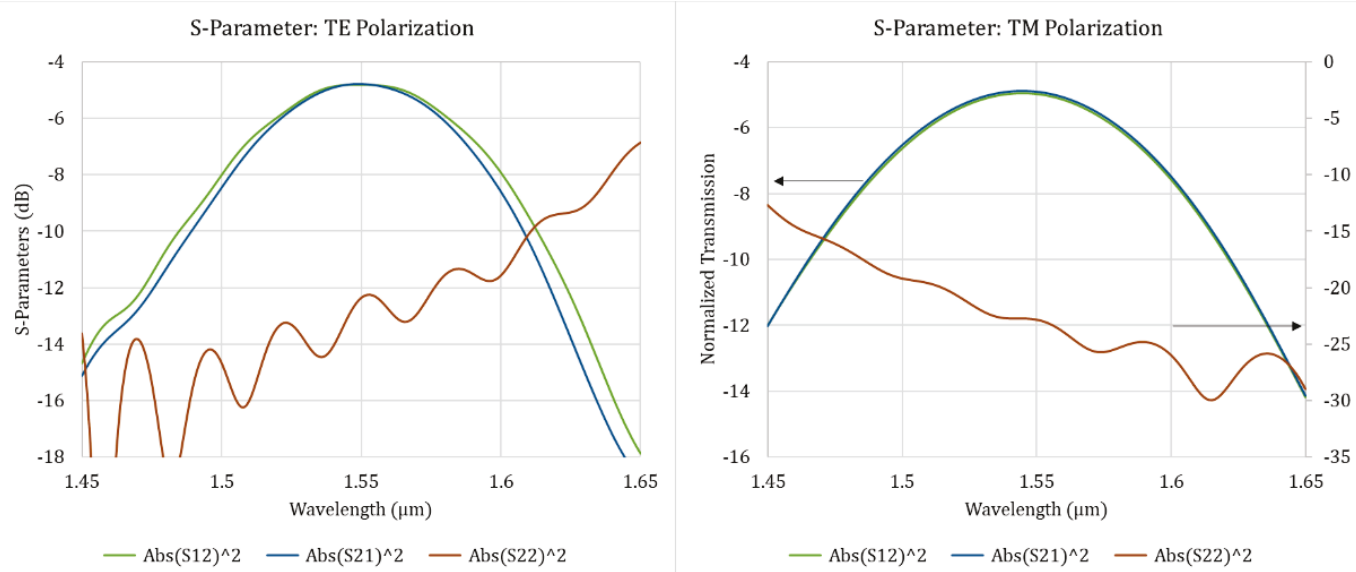

Figure 5.39. S-Parameter for the apodized SWGC for TE and TM polarization

The S-parameters of the apodized SWGC for fabrication is presented in Figure 5.39. for both TE and TM polarized modes, $S_{12} \approx S_{21}$. The back reflections are reduced to below $-10 \mathrm{~dB}$ from 1500 - $1600 \mathrm{~nm}$ for both the polarizations. Since, apodization smooths out the refractive index discontinuities, the back reflections $\left(S_{22}\right)$ in the apodized SWGC are lower in comparison to the uniform SWGC. 


\subsection{Optical Layout}

The layout of the device is created in the universal GDSII format. The optical layout for the straight uniform polarization flexible and broadband SWGC is shown in Figure 5.40. The optical layout for the focusing grating for the same uniform SWGC is depicted in Figure 5.41.

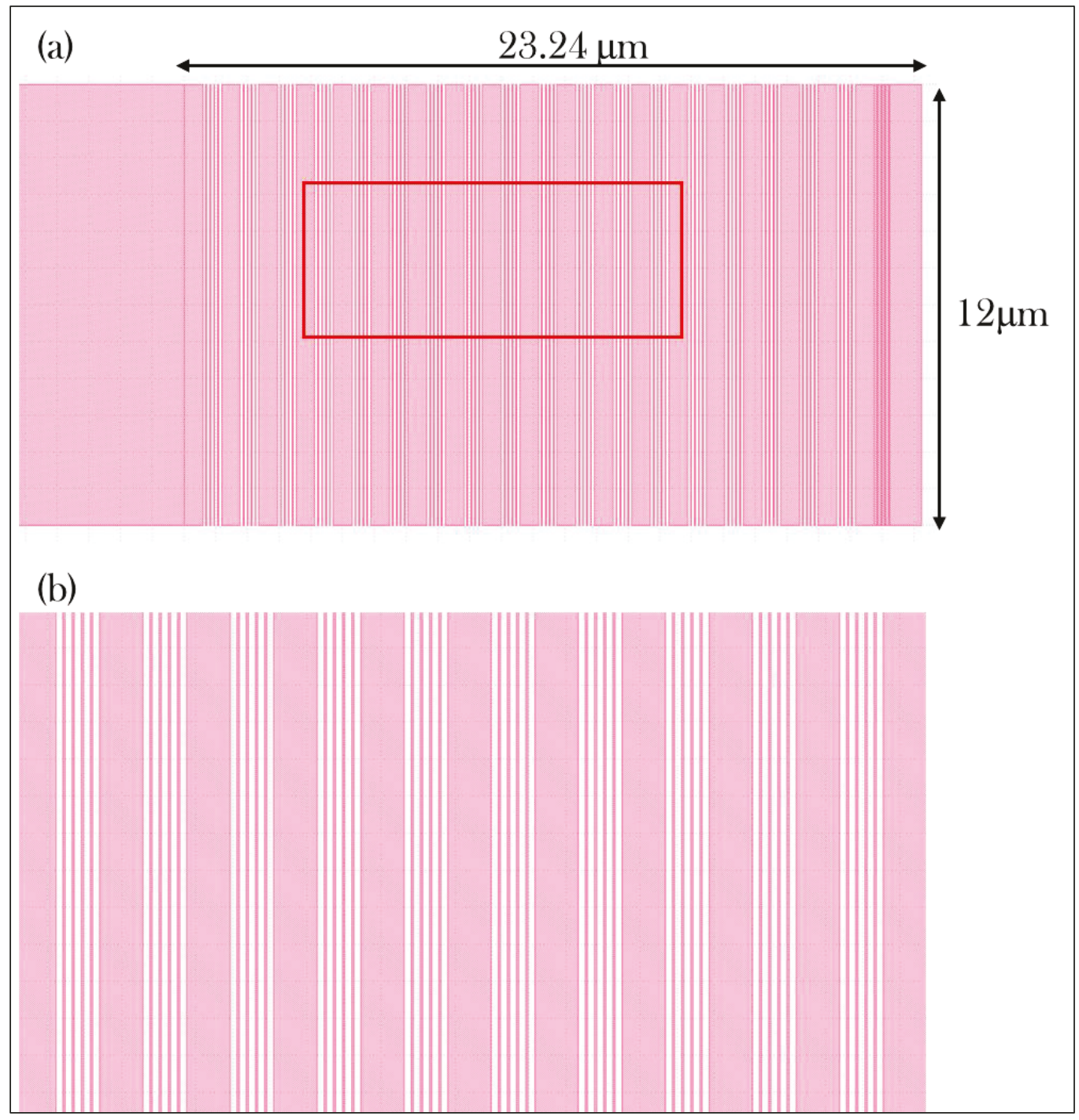

Figure 5.40. Optical layout for (a) the straight polarization flexible and broadband grating coupler; (b) Close-up view of the sub-wavelength gratings 


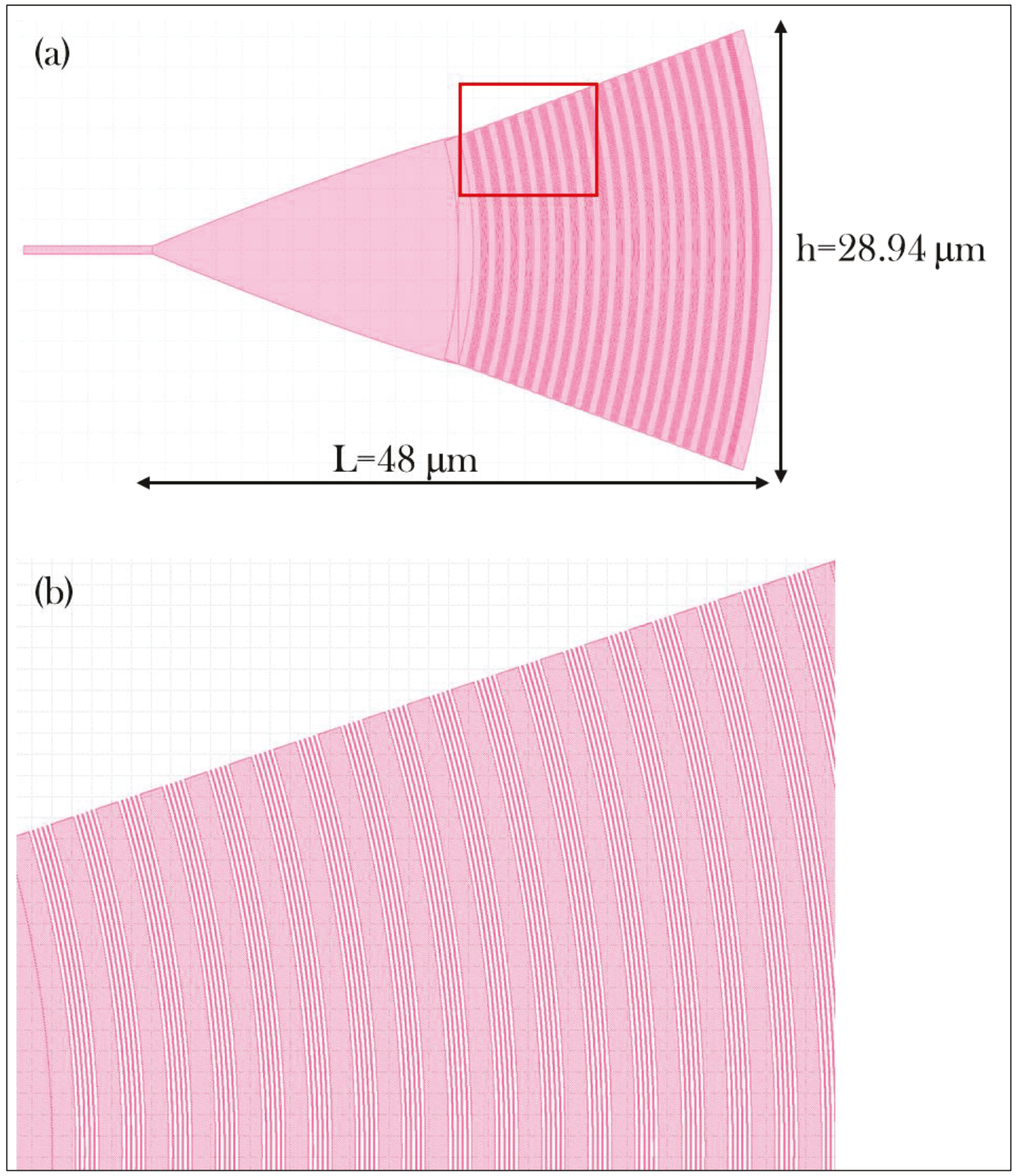

Figure 5.41. Optical layout for (a) focusing polarization flexible and broadband grating coupler; (b) Close-up view of the sub-wavelength gratings 


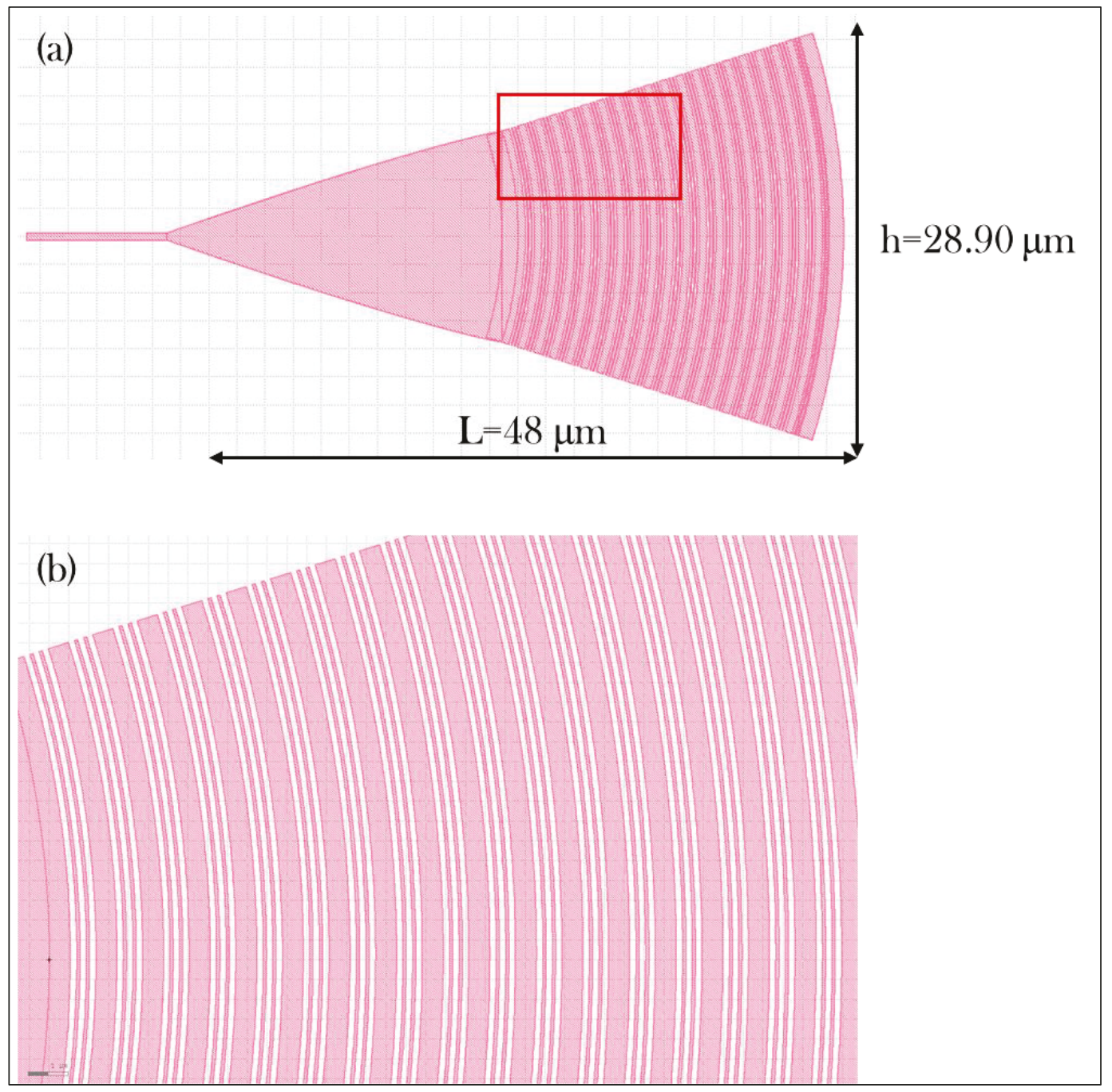

Figure 5.42. Optical layout for uniform SWGC design for fabrication (a) focusing; (b) Close-up view of the sub-wavelength gratings 


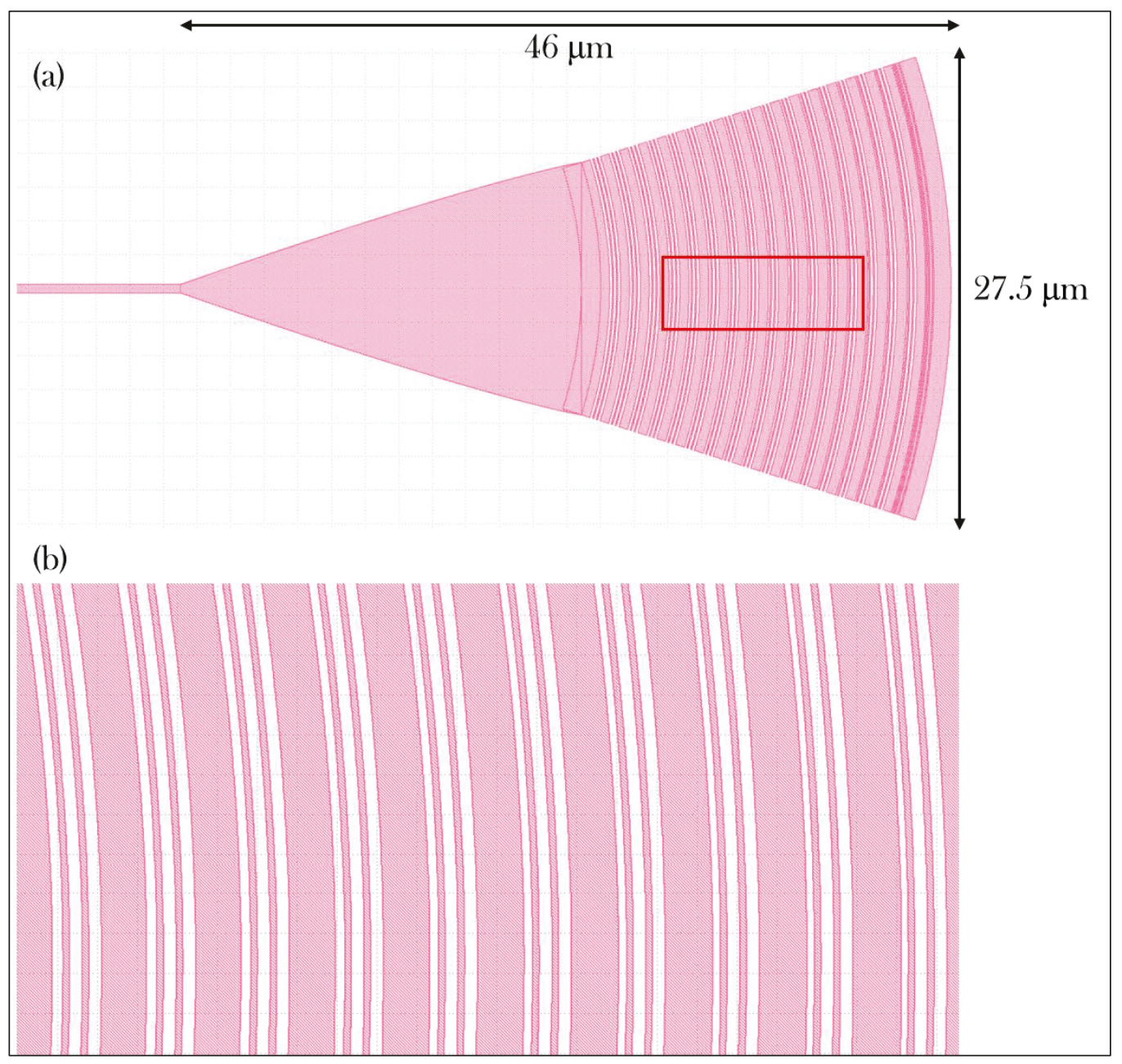

Figure 5.43. Optical layout for (a) focusing apodized polarization flexible and broadband SWGC; (b) Close-up view of the gratings 


\section{Chapter 6}

\section{Conclusions}

\section{Summary}

This thesis covered the details of design, simulation and analysis of a polarization flexible and broadband sub-wavelength grating coupler. A methodology to design a polarization flexible and broadband grating coupler was presented in this thesis. The problem statement was divided into two parts - (1) to realize a polarization flexible grating coupler and (2) to make the operation broadband. We first investigated the state-of-the-art grating couplers. The polarization independent grating couplers already reported in literature were mostly designed for a thicker SOI, because a thicker silicon layer reduces form birefringence while also resulting in an overall increase in coupling efficiency. In 2015, Song et al. [35], designed and fabricated the first polarization independent grating coupler based on a $220 \mathrm{~nm}$ SOI with a maximum simulated $\mathrm{CE}$ of $-5.4 \mathrm{~dB}$. While most of the polarization independent grating couplers exhibited a 3-dB bandwidth of $\sim 70 \mathrm{~nm}$ and 1-dB bandwidths of $\sim 40 \mathrm{~nm}$, none of them exhibited a broadband operation. To design a grating coupler that was both polarization independent and broadband it was crucial to have an in-depth knowledge of the fundamentals of the grating coupler, which are well described by the Bragg condition. The Bragg condition must be full-filled for both TE and TM polarization to effectively couple both the polarizations into the 
planar waveguide. We further delved deeper into the concept of focussing and detuned gratings, by the use of which the overall area on wafer as well as the backreflections could be reduced. To understand the factors governing the bandwidth of the grating we analysed the Bragg condition and concluded that an increase in bandwidth could be achieved by decreasing in the average effective index of the grating structure and increasing in the grating period. Reduction of the effective index is accompanied by a drop of the coupling efficiency. Xiao et al. also proposed that the bandwidth is also dependent on the fiber parameters, hence a lower a smaller beam waist can lead to an increased bandwidth without affecting the coupling efficiency. But for simulation purpose the core diameter of the incident fiber was fixed at $8.2 \mu \mathrm{m}$. Along with the theoretical knowledge it was also imperative to familiarize ourselves with the available numerical tools - the Effective Index Approximation to calculate the index of the propagating mode, the Effective Medium Theory to calculate the index of the mode in sub-wavelength structures and FDTD method for simulation of the grating structure, all of which were highly conducive for the entire design and research process. These numerical methods helped in the improvisation of the grating performance through optimization of grating parameters and analysis of the simulation results. During this process, we studied and analysed the effects of the tunable grating parameters on the coupler performance- the central wavelength and maximum coupling efficiency. We studied the effect of the grating period, the etch depth and the fill-factor. We observed that an increased grating period red-shifted the central coupling wavelength while also reducing the maximum CE for TE and TM polarizations. The etch-depth played a significant role in defining 
the grating strength of a partially etched grating coupler. For etch depth, $e d=30 \mathrm{~nm}$ the grating strength is very low and hence, the CE was reduced to almost $-8.23 \mathrm{~dB}$, whereas the maximum coupling efficiency was observed at $e d=70 \mathrm{~nm}$. An increase in the etch-depth also resulted in the blue-shift of the central wavelength as opposed to the red-shift caused by an increased grating period or fill-factor. A maximum CE was observed at fill-factor, $f f \sim 50 \%$ for the both, TE and TM polarization. Therefore, with the optimized grating parameters for an optical fiber incident at $17.4^{\circ}$ the TE and the TM polarized modes are coupled in opposite direction. A maximum simulated $\mathrm{CE}$ of $-2.94 \mathrm{~dB}$ and $-3.62 \mathrm{~dB}$ were attained for $\mathrm{TE}$ and TM polarization respectively. A 1-dB bandwidth of $45 \mathrm{~nm}$ was attained for TE, while TM depicted a 1-dB bandwidth of $37.65 \mathrm{~nm}$. The $3-\mathrm{dB}$ bandwidth of the TE reached up to $\sim 80$ $\mathrm{nm}$, whereas the TM displayed a coupling bandwidth of about $65 \mathrm{~nm}$. This design of polarization independent design was inspired by the methodology proposed by Tang and Dai in 2009. [14] The bandwidth of the partially etched polarization independent grating coupler was still not adequate. Hence, to further increase the coupling bandwidth, we must decrease the overall effective index of the grating coupler.

While reviewing state-of-the-art grating couplers, we gained insight into the sophisticated design techniques for refractive index engineering through subwavelength structures and apodization. SWGs are perceived as homogenous medium by the propagating mode and therefore, does not observe the periodic refractive index discontinuities. To further reduce these discontinuities the SWGC 
could be apodized. Apodization helps in varying the grating strength along the grating by varying the effective index of each grating period.

To convert the partially-etched grating coupler into a full-etched SWGC, the average effective index of the $2^{\text {nd }}$ mode i.e. the TM polarized mode of the polarization independent grating coupler was equated to Rytov's formula for TM polarized light in sub-wavelength periodic media. Using this technique, we found a range of values of the duty -cycle of the low and the high-index regions $\mathrm{ff}_{\mathrm{L}}$ and $\mathrm{ff}_{\mathrm{H}}$ which satisfied the average effective index. For $\mathrm{ff}_{\mathrm{H}}=1$, which represents the un-etched silicon of the partially etched grating coupler, the fill-factor of the low-index region was determined to be approximately $40 \%$. Simulations of the initial polarization independent SWGC showed lower CE for both polarizations. This structure was further optimized to attain higher $\mathrm{CE}$ and bandwidth. The grating period, duty-cycle of the grating, fillfactor of the low index region and the number of periods in the low index region were altered to achieve the desired broadband and polarization behavior. The maximum $\mathrm{CE}$ along with broadband behavior and lowest PDL was obtained for $\Lambda=$ $1175 \mathrm{~nm}, \mathrm{ff}=50 \%, \mathrm{ffL}=20 \%, \mathrm{ffH}=100 \%$ and $\mathrm{N}_{\mathrm{L}}=4$. A maximum simulated $\mathrm{CE}$ of $-4.88 \mathrm{~dB}$ and $-4.86 \mathrm{~dB}$ was attained for the TE and TM polarizations, respectively, with zero PDL at $1550 \mathrm{~nm}$. To attain maximum CE for TE and TM the fiber must be incident at an angle of $39^{\circ}$ and $16^{\circ}$ respectively. The simulated $1-\mathrm{dB}$ bandwidth demonstrated by this SWGC for the TE was increased up to $57 \mathrm{~nm}$ whereas for the TM polarized light it reached up to approx. $70 \mathrm{~nm}$. The simulated 3-dB bandwidths for both the polarizations were greater than $100 \mathrm{~nm}$. TE polarization exhibited 3-dB bandwidth of $105 \mathrm{~nm}$ whereas, TM depicted an even higher 3-dB coupling 
bandwidth of almost $121 \mathrm{~nm}$. The back-reflections were reduced to $<-10 \mathrm{~dB}$ for $\mathrm{TE}$ and $<-20 \mathrm{~dB}$ for $\mathrm{TM}$ at $1550 \mathrm{~nm}$.

Once the SWGC design was finalised we then focussed our attention towards fabrication. To meet the in-house fabrication requirements, the SWGC design was modified such that the minimum feature size is $>75 \mathrm{~nm}$. The smallest sub-wavelength structure in the polarization flexible and broadband SWGC designed for fabrication was $\sim 80 \mathrm{~nm}$. This resulted in reduced coupling bandwidth for the TE polarization and a lower coupling efficiency for TM polarized light. But these degradations were overcome through linear apodization of the SWGC. Apodization of the uniform SWGC was successful in increasing the coupling efficiency and operating bandwidth for TM and TE polarizations, respectively, while also reducing the overall backreflections by impedance matching. The back-reflections at $1550 \mathrm{~nm}$ were reduced to $<-12 \mathrm{~dB}$ for TE and $<-23 \mathrm{~dB}$ for TM. The back-reflections for both TE and TM were reduced to $<-10 \mathrm{~dB}$ across the transmission spectrum from $1500-1600 \mathrm{~nm}$.

The optical layouts for the original polarization flexible and broadband SWGC, the uniform SWGC designed for fabrication and the apodized SWGC are were also created in GDSII format. The taper that converts the $12 \mu \mathrm{m}$ wide grating coupler into a $500 \times 200 \mathrm{~nm}^{2}$ must be adiabatic and not incur considerable losses. $100 \%$ adiabatic tapers are practically impossible and hence tapers introduce some losses while converting the mode size. These tapers can be either symmetric/ asymmetric or continuous or discontinuous. For our focussing design, the maximum taper efficiency for TE is $93 \%$ and for TM its $97 \%$ across 1450 - $1650 \mathrm{~nm}$ range. 


\section{Impact Statement}

We presented through simulations the use and benefits of apodized 1-D subwavelength gratings for engineering the average effective index of the grating to support both TE and TM polarized modes while exhibiting a broadband operation, with low back reflections. The proposed SWGC is exceptional with the lowest ever reported PDL of $0.00376 \mathrm{~dB}$ at $1550 \mathrm{~nm}$. The maximum simulated coupling efficiencies of $-4.86 \mathrm{~dB}$ and $-4.88 \mathrm{~dB}$ were attained for the TE and TM polarization, respectively with back reflection as low as $-20 \mathrm{~dB}$. Compared to the existing polarization insensitive grating coupler designs, our device demonstrates a much superior broadband performance. Instead of the typical 60-70 $\mathrm{nm}$ bandwidth range we obtained the 3-dB coupling bandwidths of $105 \mathrm{~nm}$, for TE and $121 \mathrm{~nm}$, for TM. In comparison to the coupling bandwidths reported for the polarization independent design for the $460 \mathrm{~nm}$ process in [13], our design offers an increase of 1-dB coupling bandwidth by a factor of 1.9 and 1.72 times for TE and TM polarization, respectively. In comparison to the polarization independent grating coupler designed in the 220 nm process by Song et al. our designed shows an improved 1-dB coupling bandwidth by $1.54 \mathrm{X}$ for TE and 1.74X for TM. Along with the improved coupling bandwidth, our design also depicts an increased $\mathrm{CE}$ of more than $2 \mathrm{~dB}$ with respect to the design proposed by Song et al. The 3-dB bandwidths obtained also show an enormous improvement over the previously published results. The relatively large operating bandwidth makes our design useful for polarization diverse DWDM applications. In addition to the excellent potential for delivering high performance coupling, another major advantage of our proposed design is the simplicity of the fabrication: only one 
full-etch step is required, which can be done at the same etch step as defining the silicon waveguides. This makes the design highly desirable for commercial applications. The same design was also converted into a focusing grating lines which further reduced the device footprint on an SOI. Owing to the performance characteristics and ease of fabrication, this polarization flexible and broadband grating coupler design has a wide range of potential practical applications.

\section{Achievements and Publications}

1. N. Purwaha and W. N. Ye, "Broadband polarization independent grating coupler based on apodized subwavelength gratings," US Patent Application \#: 62716461, August 9, 2018.

2. N. Purwaha, A. Atieh, W. N. Ye, "Broadband and Polarization Independent SOI Grating Coupler Based on Sub-Wavelength Gratings with Low Back Reflections," submitted to OSA Continuum.

3. N. Purwaha, C. Bonfil, S. Sargheini, A. Atieh, W.N. Ye, "Broadband SOI grating coupler based on apodized sub-wavelength gratings with low back reflection for fiber-to-chip coupling of DWDM signals," invited poster presentation at Integrated Optics: Devices, Materials, and Technologies XXIII, Photonics West 2019. 


\section{Future Work}

Overall, this thesis provides a significant contribution to the field of optical I/O's by presenting a broadband and polarization flexible grating coupler. In the near future, we must conduct the experimental verification of the apodized 1D-SWGC design. Further simulations must be targeted at increasing the coupling efficiency of the grating couplers without decreasing the coupling bandwidth. This could be achieved by the use of bottom Bragg reflectors. Another area to be explored is the various apodization schemes for impedance matching and improving overlap integral of the radiated and the gaussian beam of the fiber. We have used only linear apodization in this design. The other apodization envelopes are- Cosine, Gaussian, Tanh, Sinc etc. Each of these apodization functions is expected to yield a slightly different transmission spectrum and back-reflection for both TE and TM polarization.

Another area worth exploring is the use of 2D- gratings to attain polarization independence. Zhong et al. [12] demonstrated the use of curved 2D gratings, that demonstrated exceptional broadband results $(1 \mathrm{~dB}$ bandwidth $>100 \mathrm{~nm})$ for the $\mathrm{TE}$ polarized mode with low back-reflections. These curved 2D SWGs further smoothed the refractive index discontinuities. We could examine these structures for their transmission response to TM polarized mode and design a 2D grating with curved SWGs to attain polarization independence and even higher bandwidths. Reducing the refractive index discontinuity can further reduce the back reflections. 
The taper design for conversion of the $12 \mu \mathrm{m}$ mode into a $500 \mathrm{~nm}$ mode is another area to work upon. These tapers are polarization dependent and hence add additional losses while converting the mode size. A truly polarization independent PIC must also possess a taper which is adiabatic for both TE and TM polarizations. The taper used for the optical layout exhibited a maximum transmission of 91.04\% for TM and $92.33 \%$ for $\mathrm{TE}$ at $1550 \mathrm{~nm}$. This taper experience maximum transmission efficiency of $-0.17 \mathrm{~dB}$ at $1500 \mathrm{~nm}$ and minimum transmission efficiency of $-0.8 \mathrm{~dB}$ at $1600 \mathrm{~nm}$. The drop across the wavelength range is undesirable. Therefore, a polarization independent taper where the efficiency remains majorly constant must be designed. 


\section{Bibliography}

[1] Hornsteiner, A. (2017). Fiber Optic Technology Trends in Data Transmission: Digitalization of data advance the need for constant upgrading of data networks. Optik \& Photonik, 12(4), 20-24.

[2] Kartalopoulos, S. V. (2000). Introduction to DWDM technology: data in a rainbow (pp. 56-57). SPIE Optical Engineering Press.

[3] Kopp, C., Bernabe, S., Bakir, B. B., Fedeli, J. M., Orobtchouk, R., Schrank, F., ... \& Tekin, T. (2011). Silicon photonic circuits: on-CMOS integration, fiber optical coupling, and packaging. IEEE Journal of Selected Topics in Quantum Electronics, $17(3)$, 498-509.

[4] Bakir, B. B., De Gyves, A. V., Orobtchouk, R., Lyan, P., Porzier, C., Roman, A., \& Fedeli, J. M. (2010). Low-Loss $(<1 \mathrm{~dB})$ and Polarization-Insensitive Edge Fiber Couplers Fabricated on 200-mm Silicon-on-Insulator Wafers. IEEE Photonics Technology Letters, 22(11), 739-741.

[5] Dewanjee, A., Caspers, J. N., James, D. F., \& Mojahedi, M. (2014, October). A low-loss, compact, broadband, polarization insensitive edge coupler for silicon photonics. In Photonics Conference (IPC), 2014 IEEE (pp. 560-561). IEEE.

[6] Cardenas, J., Poitras, C. B., Luke, K., Luo, L. W., Morton, P. A., \& Lipson, M. (2014). High coupling efficiency etched facet tapers in silicon waveguides. IEEE Photon. Technol. Lett., 26(23), 2380-2382.

[7] Wang, J., Xuan, Y., Lee, C., Niu, B., Liu, L., Liu, G. N., \& Qi, M. (2016, March). Low-loss and misalignment-tolerant fiber-to-chip edge coupler based on 
double-tip inverse tapers. In Optical Fiber Communications Conference and Exhibition (OFC), 2016 (pp. 1-3). IEEE.

[8] Chrostowski, L., \& Hochberg, M. (2015). Silicon Photonics Design: From Devices to Systems. Cambridge: Cambridge University Press. doi:10.1017/CBO9781316084168

[9] Andreani, L. C., Gerace, D., Passoni, M., Bozzola, A., \& Carroll, L. (2016, July). Optimizing grating couplers for silicon photonics. In Transparent Optical Networks (ICTON), 2016 18th International Conference on (pp. 1-4). IEEE.

[10]Chao Li, Huijuan Zhang, Mingbin Yu, and G. Q. Lo, "CMOS-compatible high efficiency double-etched apodized waveguide grating coupler," Opt. Express 21, 7868-7874 (2013)

[11]Yun Wang, Wei Shi, Xu Wang, Zeqin Lu, Michael Caverley, Richard Bojko, Lukas Chrostowski, and Nicolas A. F. Jaeger, "Design of broadband subwavelength grating couplers with low back reflection," Opt. Lett. 40, 4647 $4650(2015)$

[12]Qiuhang Zhong, Venkat Veerasubramanian, Yun Wang, Wei Shi, David Patel, Samir Ghosh, Alireza Samani, Lukas Chrostowski, Richard Bojko, and David V. Plant, "Focusing-curved subwavelength grating couplers for ultra-broadband silicon photonics optical interfaces," Opt. Express 22, 18224-18231 (2014)

[13]Jingjing Zhang, Junbo Yang, Huanyu Lu, Wenjun Wu, Jie Huang, and Shengli Chang, "Polarization-independent grating coupler based on silicon-on-insulator," Chin. Opt. Lett. 13, 091301- (2015) 
[14]Tang, Y., Dai, D., \& He, S. (2009). Proposal for a grating waveguide serving as both a polarization splitter and an efficient coupler for silicon-on-insulator nanophotonic circuits. IEEE Photonics Technology Letters, 21(4), 242-244.

[15]Zhenzhou Cheng and Hon Ki Tsang, "Experimental demonstration of polarization-insensitive air-cladding grating couplers for silicon-on-insulator waveguides," Opt. Lett. 39, 2206-2209 (2014)

[16]Yariv, A., \& Yeh, P. (2006). Photonics: Optical Electronics in Modern Communications (The Oxford Series in Electrical and Computer Engineering).

[17]Halir, R., Bock, P. J., Cheben, P., Ortega-Moñux, A., Alonso-Ramos, C., Schmid, J. H., ... \& Janz, S. (2015). Waveguide sub-wavelength structures: a review of principles and applications. Laser \& Photonics Reviews, 9(1), 25-49.

[18]Taillaert, D., Bienstman, P., \& Baets, R. (2004). Compact efficient broadband grating coupler for silicon-on-insulator waveguides. Optics letters, 29(23), 27492751.

[19]Xiao, Z., Liow, T. Y., Zhang, J., Shum, P., \& Luan, F. (2013). Bandwidth analysis of waveguide grating coupler. Optics express, 21(5), 5688-5700.

[20]Halir, R., Cheben, P., Janz, S., Xu, D. X., Molina-Fernández, Í., \& Wangüemert-Pérez, J. G. (2009). Waveguide grating coupler with subwavelength microstructures. Optics letters, 34(9), 1408-1410.

[21]Reed, G. T., \& Knights, A. P. (2004). Silicon photonics: an introduction. John Wiley \& Sons. 
[22]Xia Chen, Ke Xu, Zhenzhou Cheng, Christy K. Y. Fung, and Hon K. Tsang, "Wideband subwavelength gratings for coupling between silicon-on-insulator waveguides and optical fibers," Opt. Lett. 37, 3483-3485 (2012)

[23]Xiaochuan Xu, Harish Subbaraman, John Covey, David Kwong, Amir Hosseini, and Ray T. Chen, "Colorless grating couplers realized by interleaving dispersion engineered subwavelength structures," Opt. Lett. 38, 3588-3591 (2013)

[24]Ramos, C. A., MOÑUX, A. O., \& FERNÁNDEZ, Í. M. (2010). Photonic chip interconnects and integrated polarization management for coherent communication (Doctoral dissertation, PhD Thesis-Univ. de Malaga).

[25]Mekis, A., Gloeckner, S., Masini, G., Narasimha, A., Pinguet, T., Sahni, S., \& De Dobbelaere, P. (2011). A grating-coupler-enabled CMOS photonics platform. IEEE Journal of Selected Topics in Quantum Electronics, 17(3), 597608.

[26]Van Laere, F., Claes, T., Schrauwen, J., Scheerlinck, S., Bogaerts, W., Taillaert, D., ... \& Baets, R. (2007). Compact focusing grating couplers for silicon-oninsulator integrated circuits. IEEE Photonics Technology Letters, 19(21-24), 1919-1921.

[27]Waldhäusl, R., Schnabel, B., Dannberg, P., Kley, E. B., Bräuer, A., \& Karthe, W. (1997). Efficient coupling into polymer waveguides by gratings. Applied optics, 36(36), 9383-9390. 
[28]Rytov, S. (1956). Electromagnetic properties of a finely stratified medium. Soviet Physics JEPT, 2, 466-475.

[29]Lalanne, P., \& Lemercier-Lalanne, D. (1996). On the effective medium theory of subwavelength periodic structures. Journal of Modern Optics, 43(10), 20632085.

[30]Yariv, A., \& Yeh, P. (2007). Photonics-Optical Electronics in Modern Comm.

[31]Born, M. (1980). E. Wolf Principles of optics. Pergamon Press, 6, 188-189.

[32]O'Shea, D. C., Suleski, T. J., Kathman, A. D., \& Prather, D. W. (2004). Diffractive optics: design, fabrication, and test (Vol. 62). Bellingham, WA: Spie Press.

[33]Yee, K. (1966). Numerical solution of initial boundary value problems involving Maxwell's equations in isotropic media. IEEE Transactions on antennas and propagation, 14(3), 302-30

\section{[34] http://emlab.utep.edu/ee5390em21/Lecture\%2010\%20--} \%20Subwavelength\%20gratings.pdf

[35]Song, J. H., Doany, F. E., Medhin, A. K., Dupuis, N., Lee, B. G., \& Libsch, F. R. (2015). Polarization-independent nonuniform grating couplers on silicon-oninsulator. Optics letters, 40(17), 3941-3944. 


\title{
Appendix A
}

\section{Partial Etched GC Setup and 2D-FDTD}

\section{Simulation Scripts}

\author{
A.1. Fiber
}

\section{A.1.1.Properties}

\begin{tabular}{|c|c|c|}
\hline S. No. & Design Parameter & Value \\
\hline 1. & Diameter of Core & $8.2 \mu \mathrm{m}$ \\
\hline 2. & Diameter of Cladding & $125 \mu \mathrm{m}$ \\
\hline 3. & Index of Core & 1.44 \\
\hline 4. & Index of Cladding & 1.43482 \\
\hline 5 . & Angle of incidence $(\theta)$ & $17.4^{\circ}$ \\
\hline 6. & Background index & 1.45 \\
\hline 7. & Position of fiber $(x, y, z) \mu m$ & $(5,1,0)$ \\
\hline
\end{tabular}

\section{A.1.2. Setup Script}

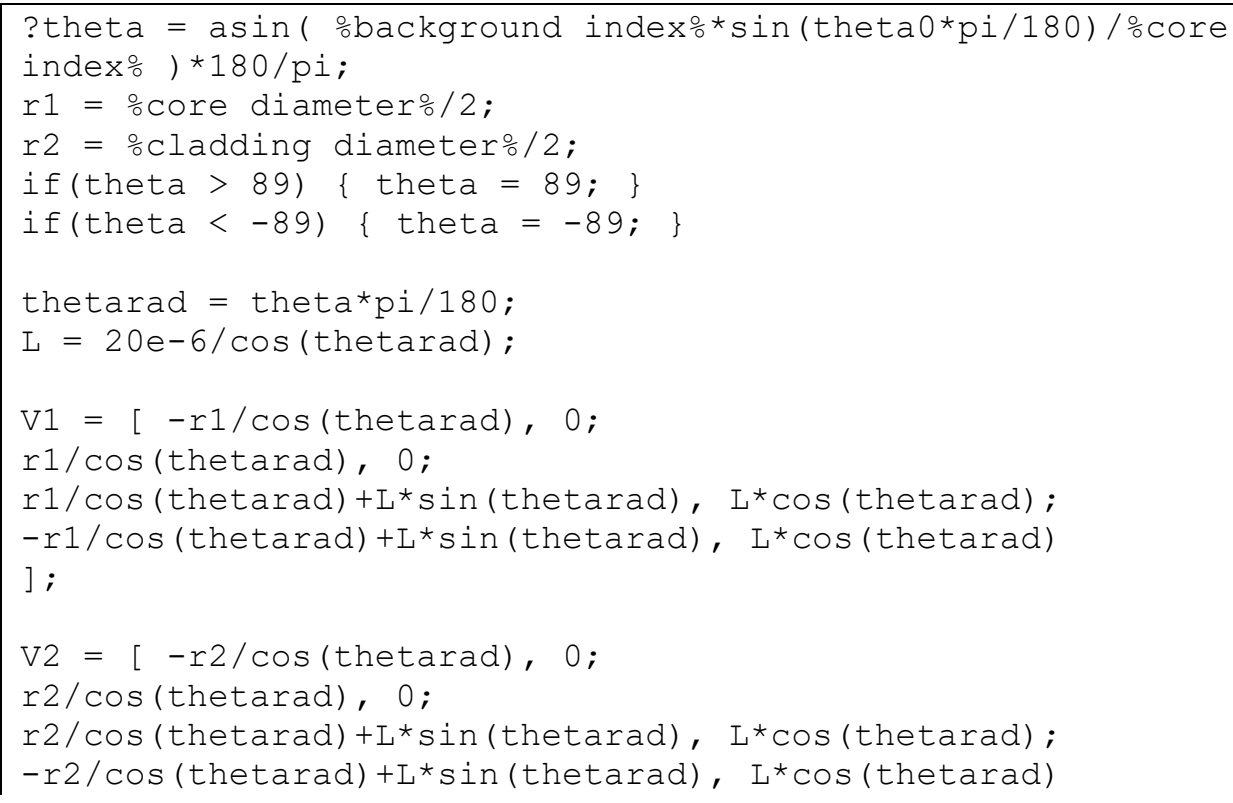




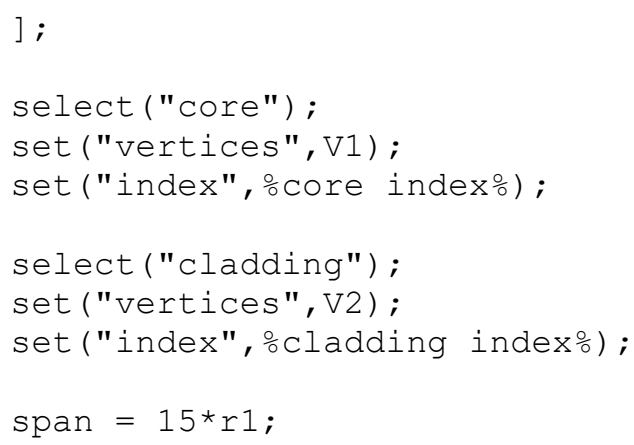

\section{A.2. Grating Coupler}

\section{A.2.1.Properties}

\begin{tabular}{|l|l|l|}
\hline S. No. & Design Parameter & Value \\
\hline $\mathbf{1 .}$ & Index & 1.4 \\
\hline $\mathbf{2}$ & Material & Si (Silicon) - Palik \\
\hline $\mathbf{3 .}$ & Target Length & $9.38 \mu \mathrm{m}$ \\
\hline $\mathbf{4 .}$ & Height total & $0.22 \mu \mathrm{m}$ \\
\hline $\mathbf{5 .}$ & Etch depth & $70 \mathrm{~nm}$ \\
\hline $\mathbf{6 .}$ & Input length & $40 \mu \mathrm{m}$ \\
\hline $\mathbf{7 .}$ & Output length & $10 \mu \mathrm{m}$ \\
\hline $\mathbf{8 .}$ & Z span & $1 \mu \mathrm{m}$ \\
\hline $\mathbf{9 .}$ & Pitch & $675 \mu \mathrm{m}$ \\
\hline $\mathbf{1 0}$ & Duty cycle & 0.6 \\
\hline $\mathbf{1 1 .}$ & Origin (x, y, z) & $(0,0,0)$ \\
\hline
\end{tabular}

\section{A.2.2.Setup Script}

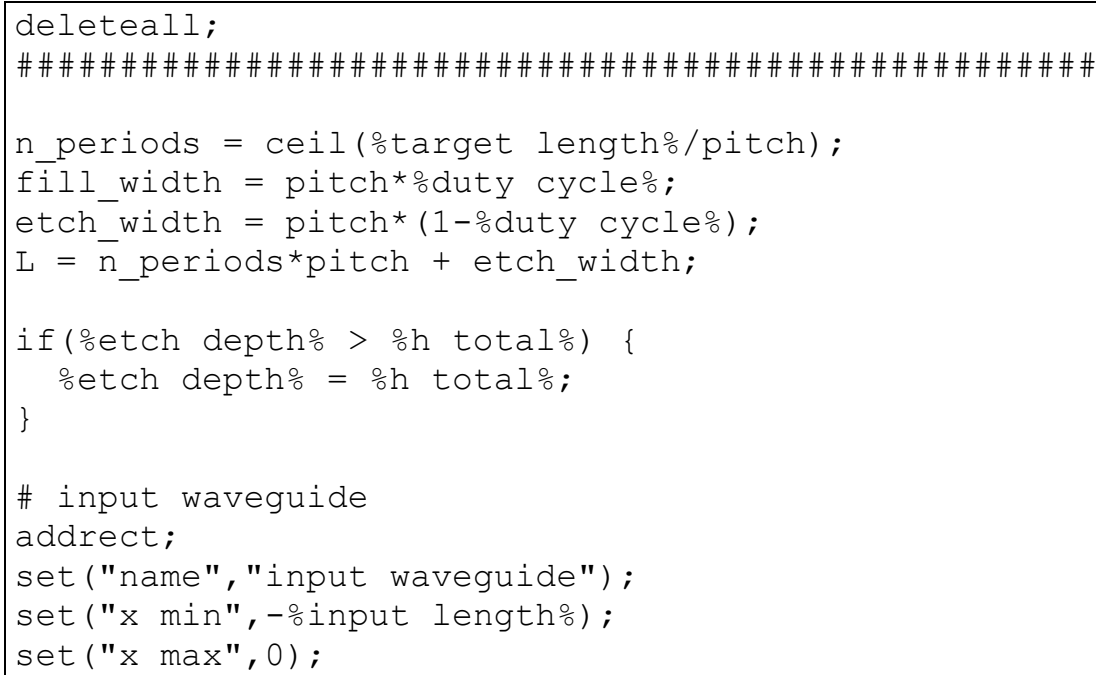




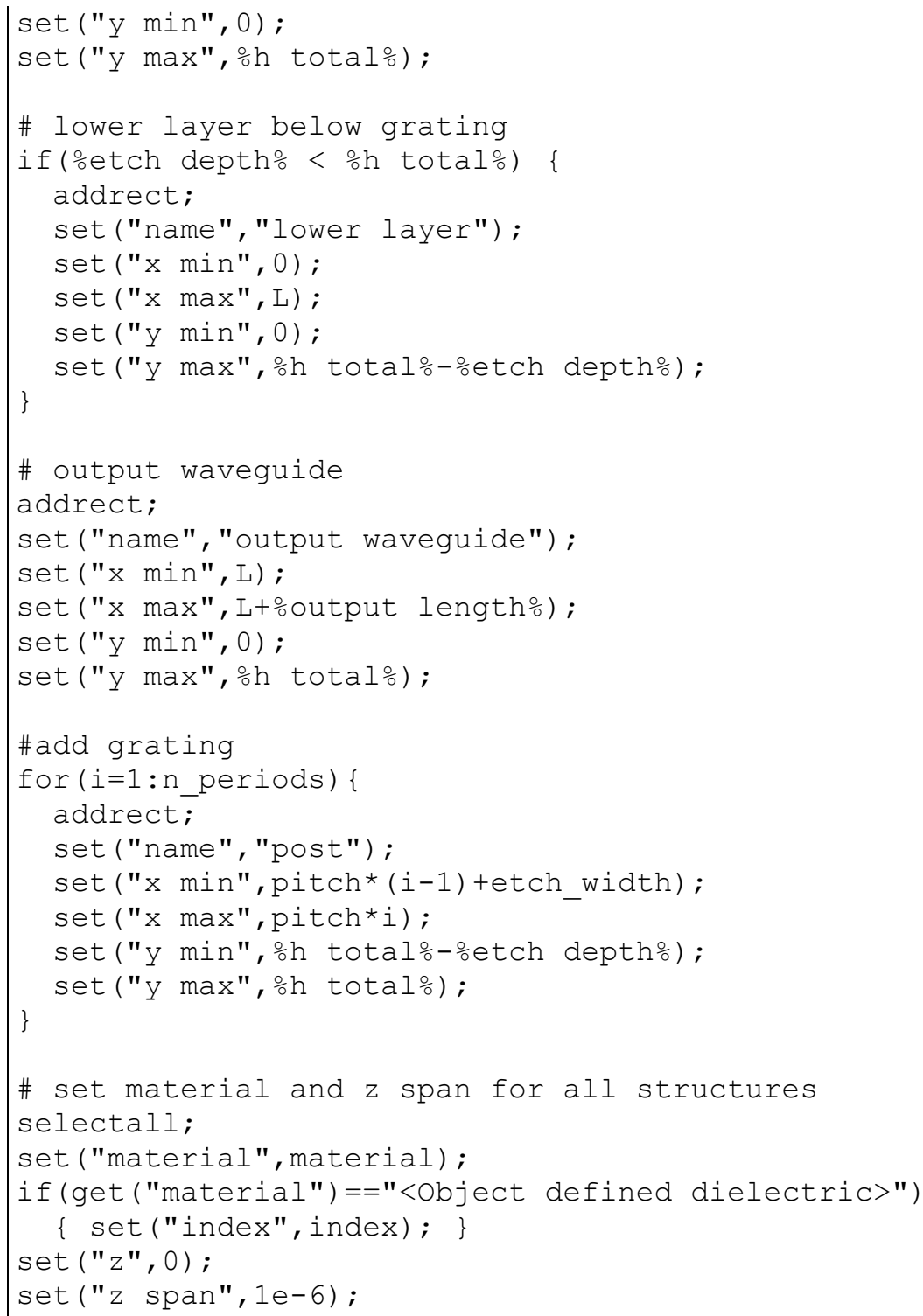

\section{A.3.Model Setup Script}

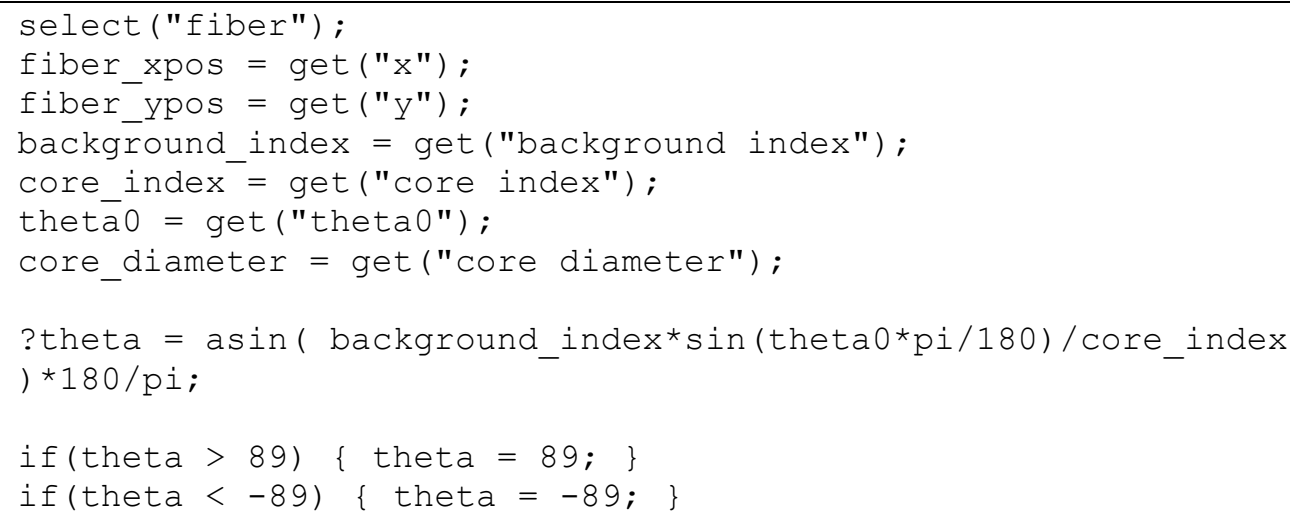


thetarad $=$ theta*pi/180;

$r 1=$ core diameter $/ 2$;

$\operatorname{span}=15^{\star} r 1 ;$

select ("FDTD: :ports: :port 1") ;

set ("x",fiber_xpos $+0.4 e-6 * \sin ($ thetarad));

set ("y",fiber_ypos $+0.2 e-6{ }^{*} \cos ($ thetarad));

\#set ("offset", span/2*tan (thetarad)) ;

set ("theta",-theta 0$)$;

if (theta $0<0)\{$

set ("offset", -span/2*tan(thetarad));

\}

else\{

set ("offset", span/2*tan(thetarad));

\} 


\title{
Appendix B
}

\section{SWGC Device Setup and 2D-FDTD}

\section{Simulation Scripts}

\author{
B.1. Fiber
}

B.1.1 Properties

\begin{tabular}{|c|c|c|}
\hline S. No. & Design Parameter & Value \\
\hline 1 . & Core Diameter & $8.2 \mu \mathrm{m}$ \\
\hline 2. & Cladding Diameter & $125 \mu \mathrm{m}$ \\
\hline 3. & Theta TE & 39 \\
\hline 4. & Theta TM & 19 \\
\hline 5. & Core index & 1.44 \\
\hline 6. & Cladding index & 1.43482 \\
\hline 7. & Background index & 1.45 \\
\hline 8. & Origin $(x, y, z)$ & $(3.75,1,0)$ \\
\hline
\end{tabular}

\section{B.1.2. Setup Script}

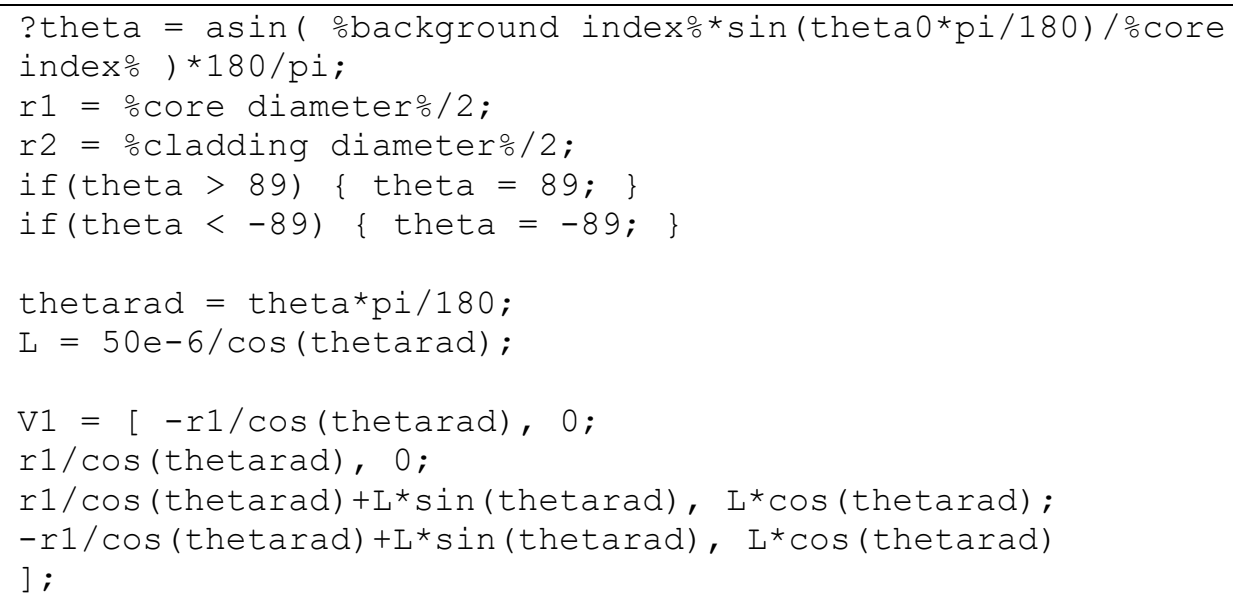




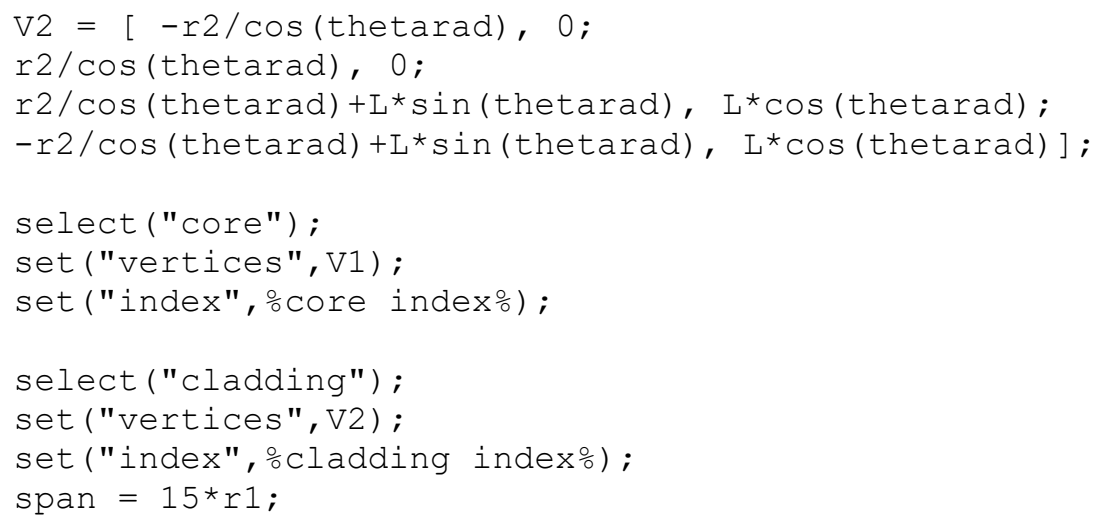

\section{B.2. Grating Coupler}

\section{B.2.1. Properties}

\begin{tabular}{|c|c|c|}
\hline S. No. & Design Parameter & Value \\
\hline 1 . & Index & 1.4 \\
\hline 2 . & Material & Si (Silicon) - Palik \\
\hline 3. & Target Length & $10.8 \mu \mathrm{m}$ \\
\hline 4. & $\mathrm{H}$ total & $0.22 \mu \mathrm{m}$ \\
\hline 5. & Etch depth & $0.22 \mu \mathrm{m}$ \\
\hline 6. & Input length & $40 \mu \mathrm{m}$ \\
\hline 7 . & Output length & $10 \mu \mathrm{m}$ \\
\hline 8. & Z span & $1 \mu \mathrm{m}$ \\
\hline 9. & Pitch & $1.175 \mu \mathrm{m}$ \\
\hline 10 & nH periods & 4 \\
\hline 11 & nL periods & 1 \\
\hline 12 & Duty cycle nL & 1 \\
\hline 13 & Duty cycle $\mathrm{nH}$ & 0.2 \\
\hline 14 & Duty cycle & 0.5 \\
\hline 15 . & Origin $(x, y, z)$ & $(0,0,0)$ \\
\hline
\end{tabular}

\section{B.2.2. Setup Script}

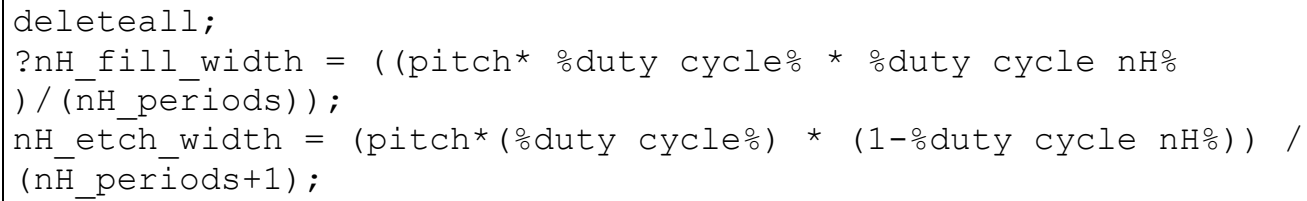




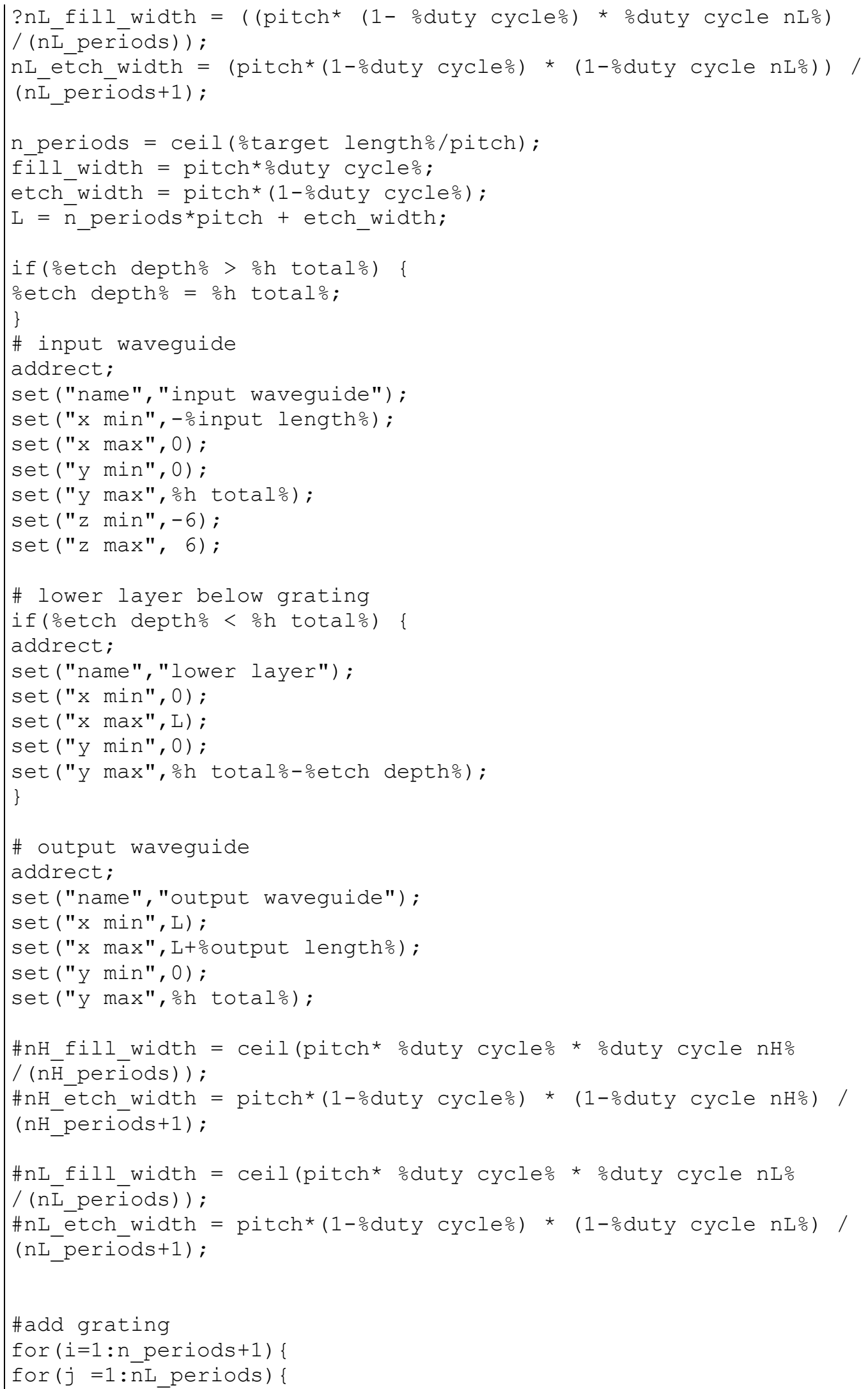




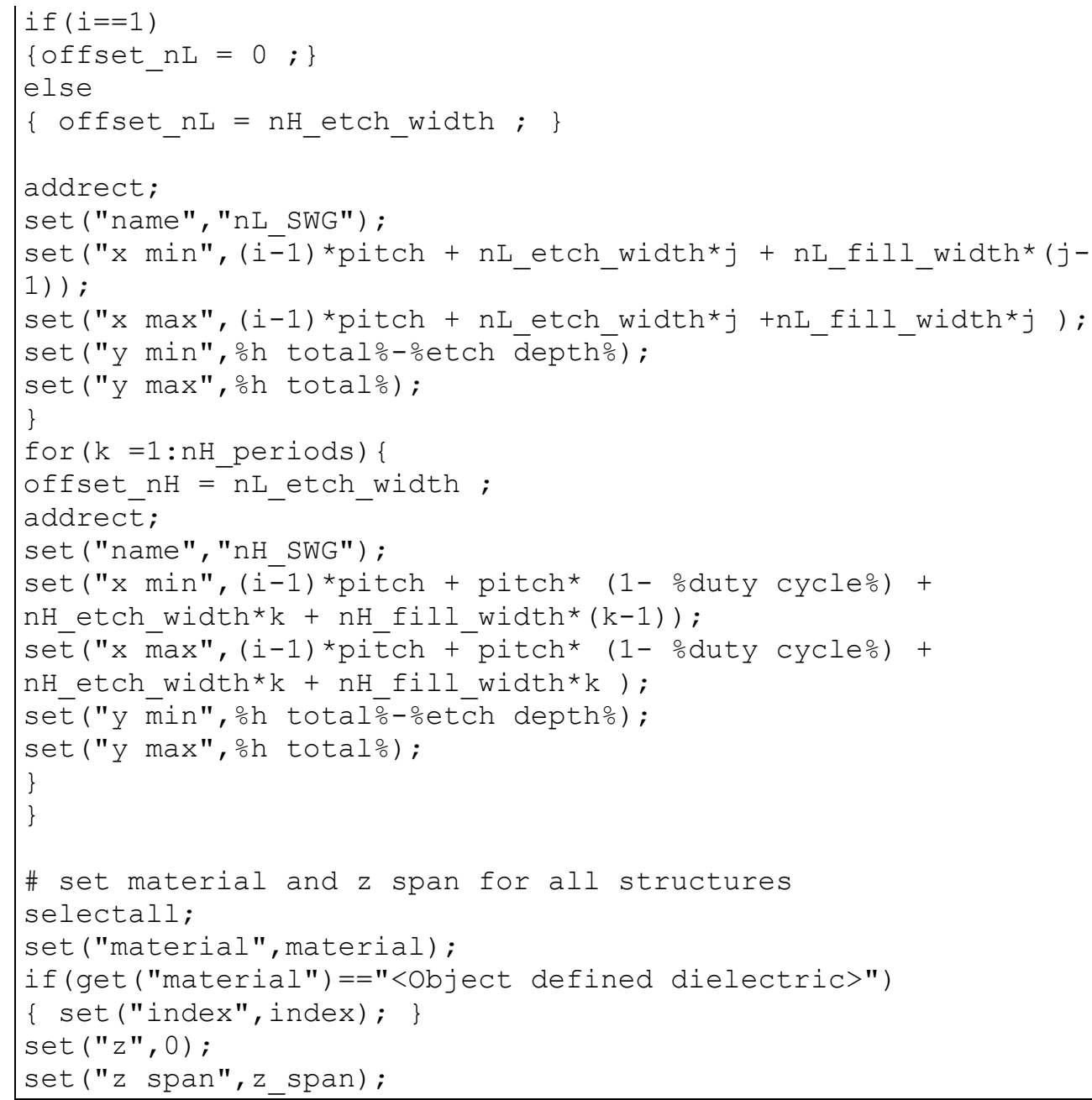

\section{B.3. Buried Oxide}

\begin{tabular}{|c|l|c|}
\hline S. No. & Parameter & Value \\
\hline $\mathbf{1 .}$ & Material & SiO2 (Glass) - Palik \\
\hline $\mathbf{2 .}$ & Thickness & $3 \mu \mathrm{m}$ \\
\hline
\end{tabular}

\section{B.4. Silicon Bottom}

\begin{tabular}{|c|l|l|}
\hline S. No. & Parameter & Value \\
\hline $\mathbf{1 .}$ & Material & Si (Silicon) - Palik \\
\hline $\mathbf{2}$. & Thickness & $7 \mu \mathrm{m}$ \\
\hline
\end{tabular}




\title{
Appendix C
}

\section{Analysis Scripts}

\author{
C.1. Model Setup Script
}

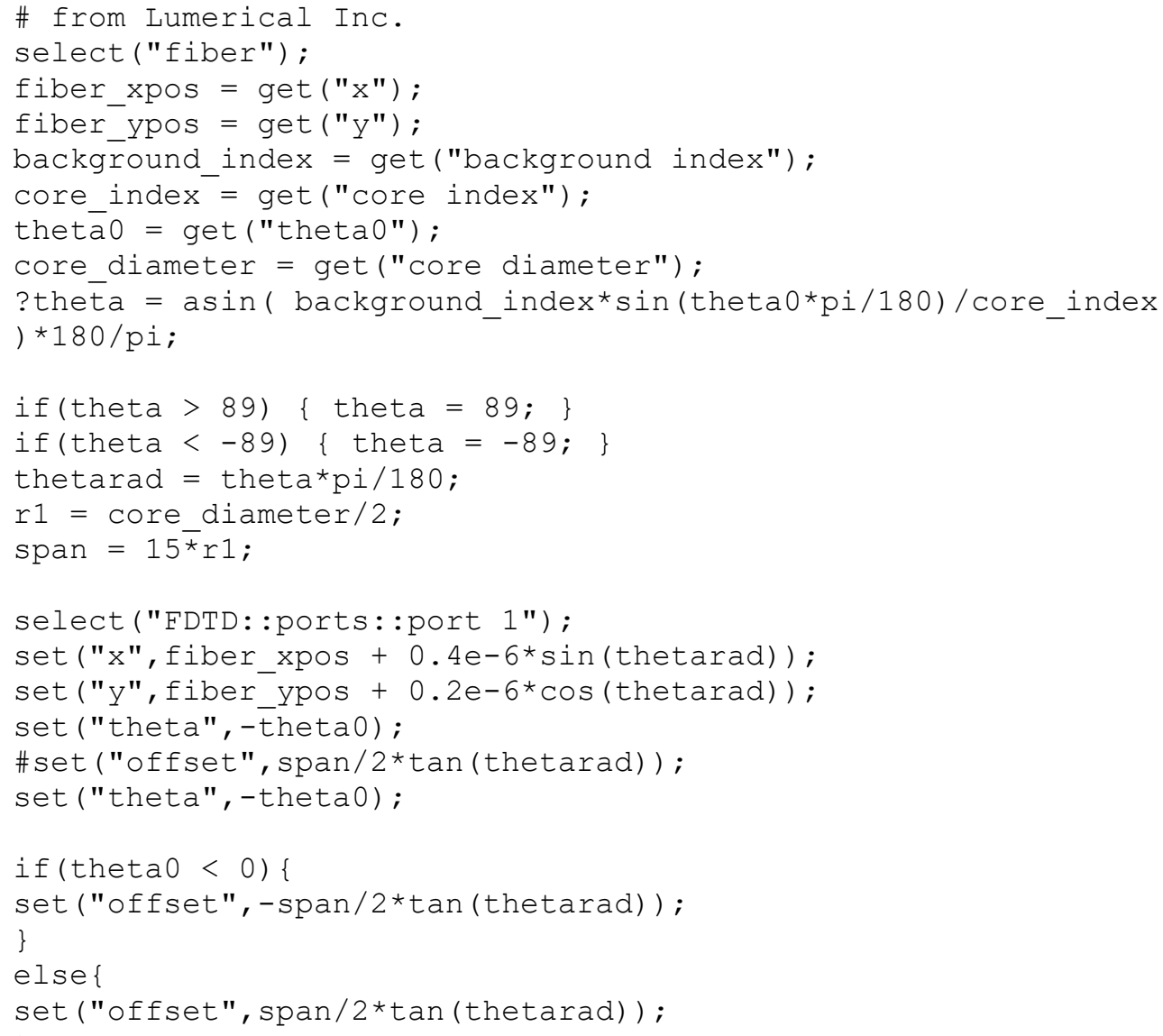

\section{C.2. Analysis Variables}

\begin{tabular}{|l|l|l|}
\hline S. No. & Parameter & Value \\
\hline 1. & Material & Si (Silicon) - Palik \\
\hline 2. & Thickness & $7 \mu \mathrm{m}$ \\
\hline 3. & Idx_1550 & 94 \\
\hline 4. & No_sample_pts & 200 \\
\hline
\end{tabular}




\section{C.3. Analysis Script}

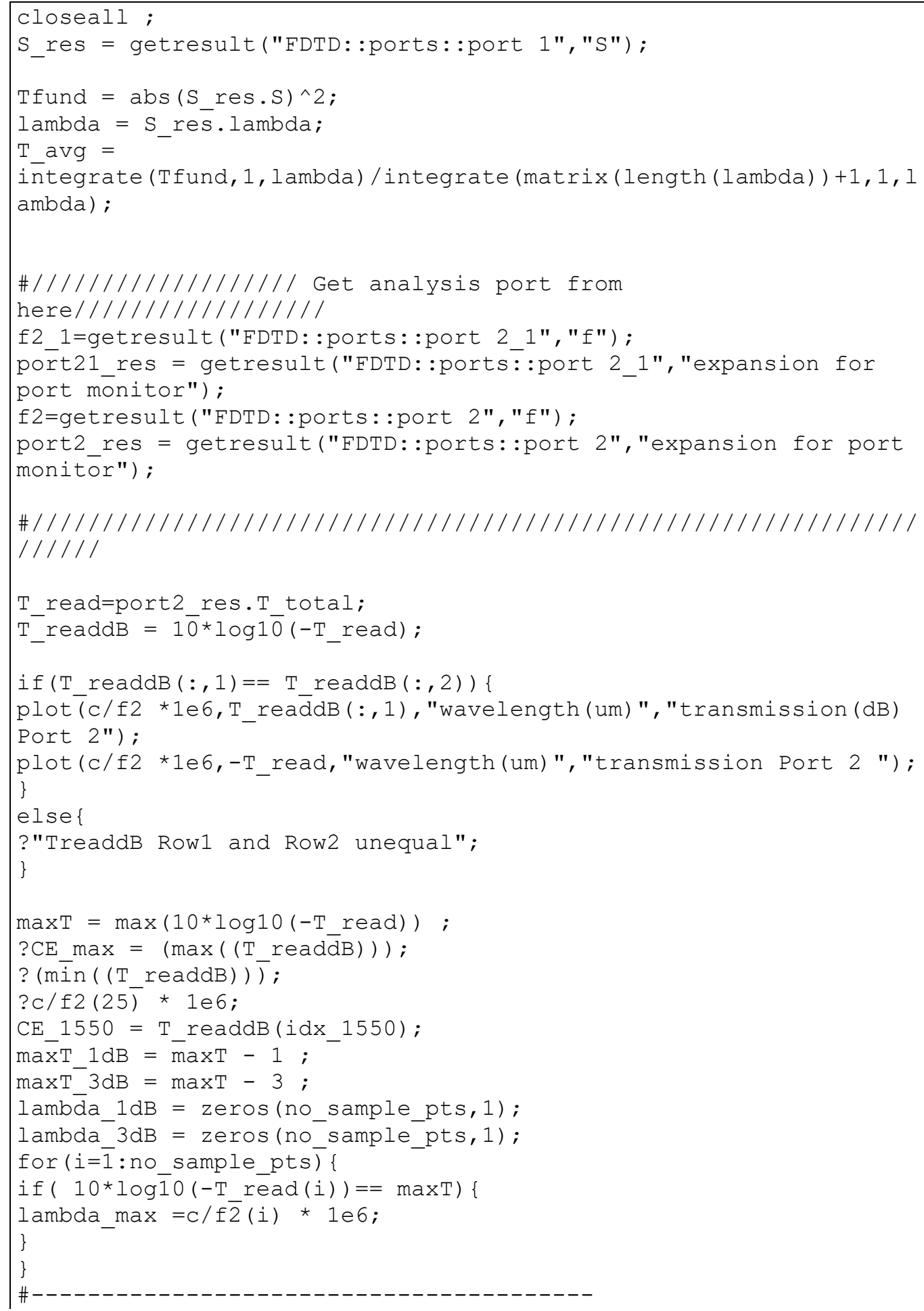




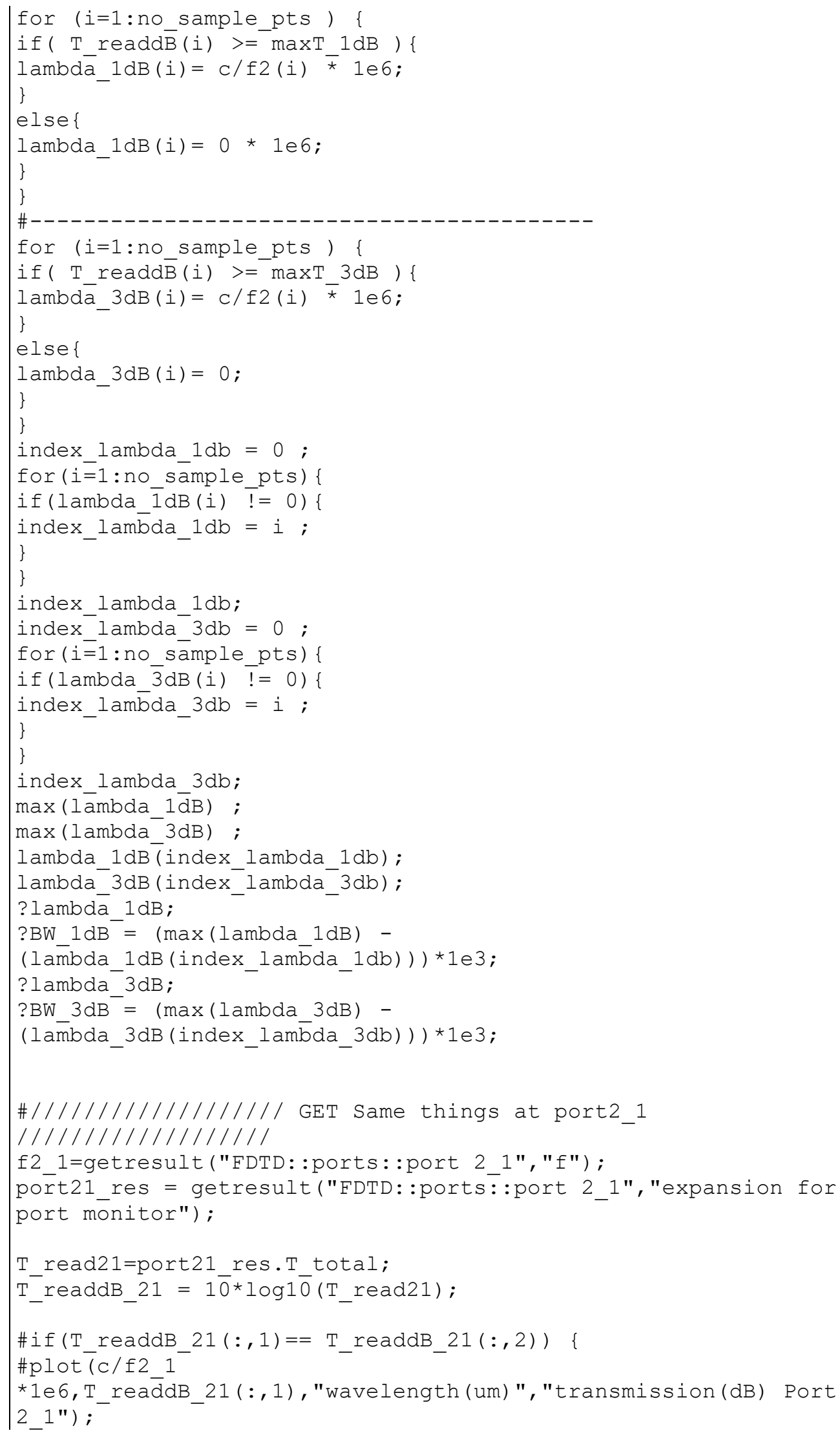




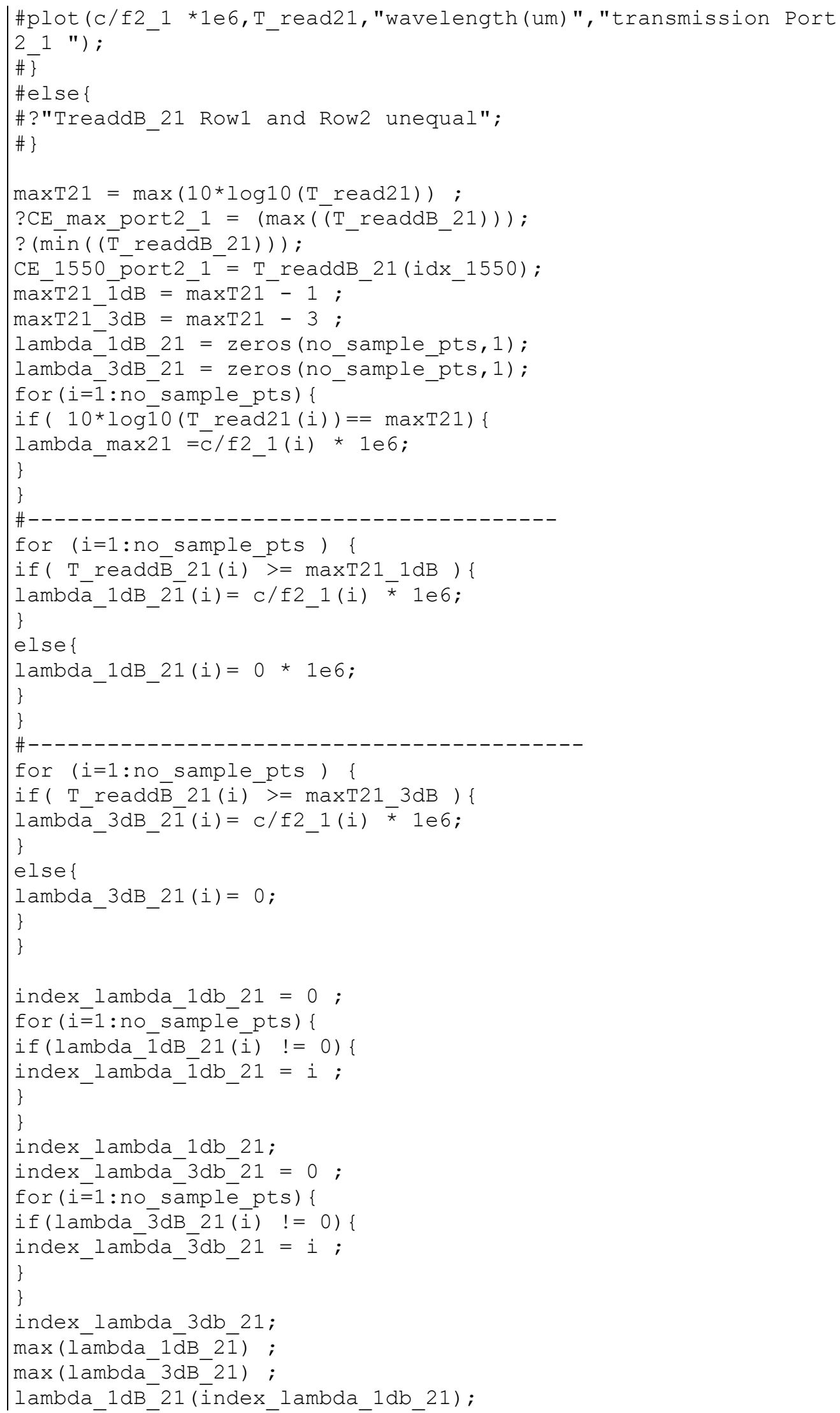




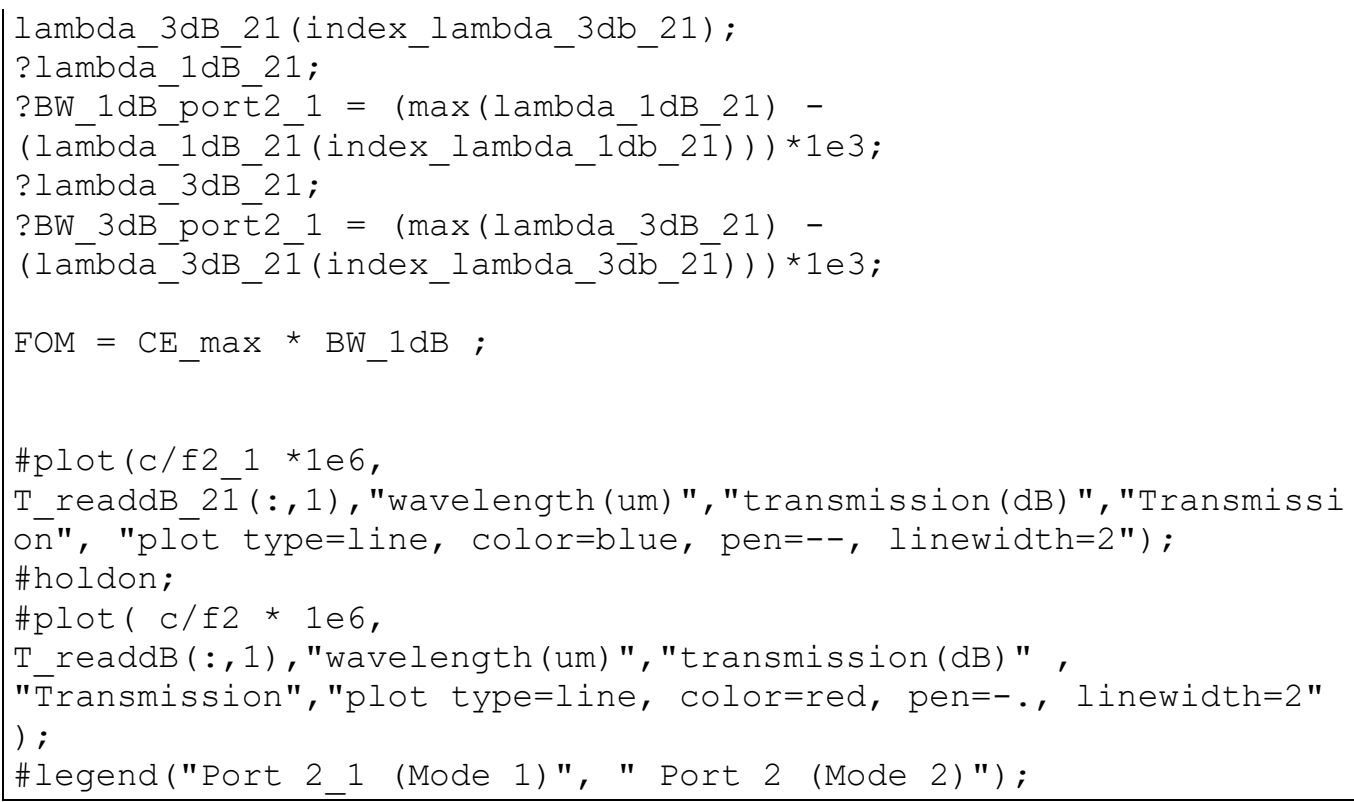

